\title{
MULTIPLE MERGING EVENTS IN THE DOUBLE CLUSTER A3128/A3125
}

\author{
James A. Rose, Alejandro E. Gaba, and Wayne A. Christiansen \\ Department of Physics and Astronomy, University of North Carolina, Chapel Hill, NC 27599 \\ Electronic mail: jim@physics.unc.edu,gaba@physics.unc.edu,wayne@physics.unc.edu \\ David S. Davis \\ Center for Space Research, Massachusetts Institute of Technology, Cambridge, MA 02139 \\ Electronic mail: dsd@space.mit.edu \\ Nelson Caldwell \\ Smithsonian Astrophysical Observatory, 60 Garden Street, Cambridge, MA 02138 \\ Electronic mail: caldwell@cfa.harvard.edu \\ Richard W. Hunstead \\ School of Physics A29, University of Sydney, NSW 2006, Australia \\ Electronic mail: rwh@physics.usyd.edu.au \\ Melanie Johnston-Hollitt \\ Department of Physics and Mathematical Physics, University of Adelaide, Adelaide SA 5005, \\ Australia and Australia Telescope National Facility, PO Box 76, Epping, NSW 1710, Australia \\ Electronic mail: mjohnsto@physics.adelaide.edu.au
}

\begin{abstract}
Multi-fiber spectroscopy has been obtained for 335 galaxies in the field of the double cluster A3128/A3125, using the 2dF multi-fiber positioner on the Anglo-Australian Telescope. When combined with previously published results, a total of 532 objects in the double cluster now have known redshifts. We have also obtained a 20 ks Chandra ACIS-I image of the central 16' x 16' of A3128 and radio imaging of the cluster with the Molonglo Observatory Synthesis Telescope and the Australia Telescope Compact Array. The spatial-kinematic distribution of redshifts in the field of A3128/A3125, when combined with the Chandra ACIS-I image of A3128, reveals a variety of substructures present in the galaxy distribution and in the hot intracluster medium (ICM). The most striking large-scale feature in the galaxy distribution is a relatively underpopulated redshift zone $\sim 4000 \mathrm{~km} \mathrm{~s}^{-1}$ on either side of the mean cluster velocity at $\sim 17500 \mathrm{~km} \mathrm{~s}^{-1}$
\end{abstract}


- We attribute this depletion zone to the effect of the extensive Horologium-Reticulum (H-R) Supercluster, within which A3128/A3125 is embedded. In addition to this largescale feature, numerous smaller groups of galaxies can be identified, particularly within the underpopulated region within $\pm 4000 \mathrm{~km} \mathrm{~s}^{-1}$ of the mean cluster redshift. Due to the large gravitational influence of the H-R Supercluster, these groups arrive at A3128 with a high infall velocity, well in excess of the local sound speed. Two of these groups appear as elongated filaments in position-velocity diagrams, indicating that they are tidally distended groups which have been disrupted after a close passage through A3128. In fact, A3125 itself appears to be in such a post passage condition. We have identified a primary NE-SW merger axis connecting A3128 with A3125, along which the filaments are also oriented. In addition, the Chandra image reveals that the X-ray emission is split into two components, each with very small core radii, that are separated by $\sim 1 \mathrm{Mpc}$ along the NE-SW axis. We have combined the redshift, X-ray, and radio data to propose that the complex X-ray morphology revealed in the Chandra image is likely the result of a hypersonic infall of a relatively small group into A3128. The group produces a major disruption in the ICM due to its high infall velocity.

Subject headings: galaxies: evolution — galaxies: elliptical and lenticular, $\mathrm{cD}$

\section{Introduction}

The distribution of groups, clusters, and superclusters of galaxies represents a fundamental testing ground for theories of the origin and evolution of structure in the universe. Until recently, the intersection between observation and theory has largely relied on statistical properties of galaxy spatial/kinematic clustering, along with X-ray determined global ICM temperatures and

azimuthally averaged radial brightness and temperature profiles. In contrast, much of the existing observational data indicates that large-scale asymmetric, probably filamentary, structures, which are not easily subjected to statistical definition on an individual basis, are present in clusters and superclusters (e.g., Gregory \& Thompson 1978; Shandarin 1983; de Lapparent, Geller, \& Huchra 1986; West, Jones, \& Forman 1995; West \& Blakeslee 2000).

Multi-fiber spectroscopy of galaxies with 400-fiber positioners deployed over $2^{\circ}$ fields, as well as the unprecedented combination of spatial resolution and sensitivity in X-rays provided by the Chandra and XMM-Newton Observatories, are rapidly advancing the observational view of clusters of galaxies. In addition, numerical simulations, carried out within the framework of a cold dark matter dominated universe in which structure is built up in a hierarchical fashion, have reecently reached the point where large areas, on scales comparable to the largest superclusters, can be simulated in some detail (Pearce et al. 2001 and references therein). Furthermore, using parametrized physics of the baryonic component, simulations within the framework of a cold dark matter dominated universe now predict the time evolution of the baryonic material (Kauffmann et al. 1999a,b; 
Somerville \& Primack 1999; Benson et al. 2001 and references therein), thus providing a more direct link to the observational data. Hence the opportunity now presents itself to make a comprehensive comparison between observations and simulations in the case of specific clusters/superclusters, thereby providing a more sensitive probe of structure evolution than can be gained from purely statistical considerations. Recent studies of the Shapley Supercluster, an extremely massive concentrations of galaxies and clusters first pointed out by Shapley (Shapley 1930; Raychaudhury 1989), represent one example of the possibilities provided by an intensive campaign of multi-fiber spectroscopy, as well as of X-ray and radio imaging. These studies have produced a wealth of data on the spatial/kinematic structures present in the Shapley Supercluster among the galaxies (e.g., Quintana et al. 2000; Drinkwater et al. 1999; Bardelli, Zucca, \& Baldi 2001) and on the hot ICM (e.g., Ettori et al. 2001), as well as results on the the emission line and radio properties of cluster galaxies (Baldi, Bardelli, \& Zucca 2001; Venturi et al. 2001). In the process, a variety of structures have been found, and the influence of environment on both galaxy and radio source evolution has been partially elucidated.

In this paper we present new observational data on a double galaxy cluster, A3128/A3125, which is itself embedded in the massive Horologium-Reticulum (H-R) Supercluster. As is discussed later, the H-R Supercluster, and the above-mentioned Shapley Supercluster, represent the two largest cluster concentrations within the local $300 \mathrm{~h}_{50}^{-1} \mathrm{Mpc}^{1}$. The observational data consist of multi-fiber spectroscopy of the double cluster A3128/A3125 with the Anglo-Australian Telescope and $2^{\circ}$ field multi-fiber positioner. In addition, we present X-ray imaging of the the central regions of A3128 and A3125 with the Chandra X-ray Observatory. Radio observations obtained with the Molonglo Observatory Synthesis Telescope and the Australia Telescope Compact Array are also presented. These observations have revealed a variety of substructures, both in galaxy groupings and in the multiple $\mathrm{X}$-ray peaks detected, which in responding to the large gravitational acceleration provided by the H-R Supercluster, reach unusually high infall velocities into A3128. Thus the connection between A3128/A3125 and the surrounding H-R Supercluster provides an opportunity to characterize substructure merging on a variety of scales and unusual conditions. In $\S 2$ we present the multi-fiber spectroscopy and X-ray and radio data. In $\S 3$ we discuss the kinematic structures revealed by the multi-fiber spectroscopy, while in $\S 4$ we discuss the observed state of the ICM, as inferred from the Chandra observations, and in $\S 5$ we present the MOST and ATCA radio imaging. A discussion of our results is given in $\S 6$; specifically, we attempt to produce a unified view of the merging events occurring in A3128/A3125 based on the full set of observations.

\footnotetext{
${ }^{1}$ Throughout this paper we adopt $H_{0}=50 h_{50}^{-1} \mathrm{~km} \mathrm{~s}^{-1}$
} 


\section{Observational Data}

\subsection{AAT/2dF Multi-Fiber Spectroscopy}

Multi-fiber spectroscopy in A3128 and A3125 (also known as DC0329-53 and DC0326-52 from the Dressler (1980) study) has been carried out using the 400 fiber positioner and double spectrograph system (2dF) on the Anglo-Australian Telescope (AAT). The field center for the observations is at 3:29:24 -52:49:45 (J2000), so as to fully cover the A3128/A3125 double cluster system with the $2^{\circ}$ field of $2 \mathrm{dF}$. We obtained a complete catalog of all galaxies down to a magnitude of $\mathrm{B}_{J}$ $<18.5$ in this field from the UKST survey plates scanned by the SuperCOSMOS machine at the Royal Observatory Edinburgh (Hambly et al. 1998, 2001). Discrimination between stars and galaxies is carried out by the SuperCOSMOS software, thus we restricted our sample to those objects identified as galaxies in the SuperCOSMOS catalog. A total of 698 galaxies to the limit $\mathrm{B}_{J}<$ 18.5 were found. However, spectroscopy of 72 galaxies has previously been obtained in the double cluster A3128/A3125 by Caldwell \& Rose (1997), and 193 galaxies have been observed in the ESO Nearby Abell Cluster Survey (ENACS; Katgert et al. 1996, 1998). Since 42 galaxies are common to Caldwell \& Rose (1997) and ENACS, a total of 223 velocities were available prior to our study. The ENACS velocities are concentrated in A3128, hence it was highly desired to increase the number of velocities in A3125 to be able to study its kinematic properties. We excluded from our SuperCOSMOS-based catalog the 72 galaxies previously observed by Caldwell \& Rose (1997; where the galaxies are referred to by their Dressler (1980) numbers), as well as 22 background and foreground galaxies from the ENACS survey. We kept the other 171 ENACS galaxies, since we desired higher $\mathrm{S} / \mathrm{N}$ spectra for them, but gave them a lower priority in the fiber assignment process. Because 42 of the Caldwell \& Rose (1997) galaxies are also in the ENACS list, those galaxies were also included in the final catalog. Altogether, the number of galaxies in the final catalog was reduced to 640 .

The AAT/2dF system has 2 identical spectrographs each receiving 200 fibers, for a total of 400 fibers (Lewis et al. 2000). To allocate the 400 fibers, including sky fibers, we used the $2 \mathrm{dF}$ configuration program CONFIGURE, which gave a configuration at the telescope allowing 371 object and 24 sky fibers (5 fibers on the A spectrograph were unusable).

The A3128/A3125 field was observed in service mode on 21 January, 2001 (UT). Five separate exposures of 30 minutes were taken, using the 1200 lines $\mathrm{mm}^{-1}$ blue grating in first order, yielding a dispersion of $1.11 \AA$ /pix at the Tek5 1024 X 1024 pixel CCD, and a spectral resolution of $\sim 2.8$ $\AA$ (or $\sim 200 \mathrm{~km} \mathrm{~s}^{-1}$ ) FWHM. The wavelength range covered is $3750 \AA-4850 \AA$. A total of 371 spectra were obtained resulting in 358 measured redshifts; of these 23 turned out to be misclassified Galactic stars. Hence we obtained velocities for a total of 335 galaxies. For 58 of these galaxies, and for two of the stars, velocities have been published previously by either the ENACS collaboration (Katgert et al. 1998) or Caldwell \& Rose (1997). Including the other previously available velocities

from Katgert et al. 1998 and Caldwell \& Rose (1997) we have in our sample redshifts for 532 galaxies in our SuperCOSMOS-derived catalog, for $76 \%$ completeness to $\mathrm{B}_{J}<18.5$. 
The data from each spectrograph were reduced independently using the $2 \mathrm{dF}$ data reduction system 2dfdr. The standard automatic reduction procedure was followed which automatically does the debiassing, trim-line map generation, fiber extraction, cosmic ray removal, wavelength calibration and sky subtraction. The reduced files from each exposure were then combined into single spectra of improved S/N.

Heliocentric radial velocities from the absorption line spectra were obtained for all galaxies using the fxcor routine in the IRAF rv radial velocity package. The spectrum of a star that was included in the sample was used as the radial velocity template. The radial velocity zero-point for that star was determined by cross-correlating its spectrum against that of several stars with known radial velocities in the Coude Feed Spectral Library (Jones 1999), which is available from the NOAO ftp archive. Where possible, radial velocities were also measured from emission line features by two of us (JAR and AEG) independently, by fitting a gaussian profile to all measurable emission lines. An average offset of $129 \mathrm{~km} \mathrm{~s}^{-1}$ between emission and absorption line velocities was found for cases where good velocities can be obtained for each. The reason for such an offset is unknown. However, uncertainties of $\sim 100 \mathrm{~km} \mathrm{~s}^{-1}$ in the radial velocities are small compared to the large systematic motions found in this paper.

A final radial velocity for each object was thus obtained using the following approach. For cases in which the emission spectrum dominates, the final velocity is based entirely on the emission line measurements with a correction of $+129 \mathrm{~km} \mathrm{~s}^{-1}$. In the case that both reliable emission and absorption velocities can be obtained (i.e., a standard old-galaxy spectrum is clearly visible underneath the emission), we average the emission and absorption measurements, after first correcting the emission measurements by $129 \mathrm{~km} \mathrm{~s}^{-1}$. In Table 2 all radial velocity measurements are listed. In columns (1) and (2) are the reference number from our $2 \mathrm{dF}$ catalog and reference numbers from previous observations respectively. This is followed by the RA and Dec, J2000, for each galaxy, the $\mathrm{B}_{J}$ magnitude, and the radial velocity. In column (7) we tabulate whether the velocity was based exclusively on emission lines ("e"), exclusively on absorption lines ("a"), or on a combination of the two ("ae"). In column (8) we give the error in the radial velocity, as given by the cross-correlation routine. Note that this error estimate is invalid for spectra whose velocity determinations are based on emission lines only. For the latter, error estimates are difficult to produce, especially since they are very dependent on the strength of the emission lines and the $\mathrm{S} / \mathrm{N}$ ratio in the spectra. In most cases, however, in which emission dominates, we have been able to locate a line with sufficient $\mathrm{S} / \mathrm{N}$ ratio that a velocity error in excess of $100 \mathrm{~km} \mathrm{~s}^{-1}$ is unlikely. Finally, in column (9) we tabulate whether any emission line is found in the spectrum.

As a test for a zero point uncertainty in our velocities we compared our $2 \mathrm{dF}$ velocities with those in common with both the ENACS (Katgert et al. 1998) and Caldwell \& Rose (1997) samples. There are 57 galaxies for which we have duplicate measures with ENACS. Omitting the two cases with large velocity discrepancies we find a mean zero point difference of $-38 \mathrm{~km} \mathrm{~s}^{-1}$ between the $\mathrm{AAT} / 2 \mathrm{dF}$ and ENACS velocities, with the $2 \mathrm{dF}$ velocities lower than ENACS. The rms dispersion between the two samples is $72 \mathrm{~km} \mathrm{~s}^{-1}$. There are also three galaxies in common between the 
2dF data and that of Caldwell \& Rose (1997), for which there is no ENACS velocity as well. Our mean $2 \mathrm{dF}$ velocity is $33 \mathrm{~km} \mathrm{~s}^{-1}$ lower than Caldwell \& Rose (1997) for those three galaxies. There are also 42 galaxies in common between Caldwell \& Rose (1997) and ENACS. The mean velocity difference is $-21 \mathrm{~km} \mathrm{~s}^{-1}$, in the sense that the Caldwell \& Rose (1997) velocities are lower than ENACS. Thus small velocity zero point errors may exist in the data, but at a level insignificant compared to the large group motions discussed in $\S 3$.

We also measured $[\mathrm{OII}] \lambda 3727$ emission line equivalent widths to compare emission line strengths for galaxies in different parts of the cluster. Unfortunately, for the fainter galaxies in the sample we encountered considerable problems in the continuum levels in the sky-subtracted spectra, especially in the blue end of the spectrum occupied by [OII] $\lambda 3727$. In many cases we find the continuum level is actually negative, leading to an unphysical negative equivalent width for $[\mathrm{OII}] \lambda 3727$. We tried to remedy the situation by correlating the SuperCOSMOS $\mathrm{B}_{J}$ magnitude with the flux in the continuum of the spectra near $4500 \AA$. Our goal was to find a mean normalization factor between the $\mathrm{B}_{J}$ magnitude and the continuum flux in the spectra for the brighter galaxies, and then use that normalization factor to allow substitution of the more reliable $\mathrm{B}_{J}$ magnitude in calculating the $[\mathrm{OII}] \lambda 3727$ equivalent width from the observed instrumental flux in the line and the blue magnitude. However, there is such a large scatter in the plotted relation between $\mathrm{B}_{J}$ magnitude and continuum flux in the spectra that we were unable to follow this plan. Thus although we are able to search to sensitive limits (typically better than $2 \AA \mathrm{FWHM}$ ) in the [OII] $\lambda 3727$ line, we are unable to produce reliable equivalent widths. As a result, we list only whether or not emission has been detected in column (9), but no quantitative information in regard to equivalent width of $[\mathrm{OII}] \lambda 3727$.

For the same 55 galaxies in common between our sample and the ENACS sample we find the following emission line statistics. For 30 galaxies both ENACS and AAT/2dF spectra show no emission lines, while for 13 galaxies both ENACS and AAT spectra record emission. In addition, there are 12 galaxies for which we find emission lines, but ENACS doesn't. There are no galaxies for which ENACS reports emission but we do not. Hence, our AAT/2dF spectra are finding emission lines at approximately twice the frequency of the ENACS spectra. To further assess this discrepancy, for two galaxies we report emission lines, ENACS does not, and Caldwell \& Rose (1997) also do not report emission. Specifically, the two galaxies are AAT\#477=ENACS\#148=CR\#80a, for which we find an equivalent width in [OII $] \lambda 3727$ of $9 \AA$, and AAT\#481=ENACS\#151=CR\#53a, for which we find an equivalent width of $6 \AA$. We have reinspected the Argus spectra obtained in Caldwell \& Rose (1997), and now see evidence for [OII] $\lambda 3727$ emission in both these spectra. Both Argus spectra in Caldwell \& Rose (1997) are clearly of substantially lower S/N ratio in the vicinity of $[\mathrm{OII}] \lambda 3727$, while the AAT $/ 2 \mathrm{dF}$ detections are robust. A comparison between the $2 \mathrm{dF}$ and Argus spectra of the two galaxies is made in Fig. 1, where the clear detection of [OII] $\lambda 3727$ emission in the $2 \mathrm{dF}$ spectra is evident. In short, we conclude that the better $\mathrm{S} / \mathrm{N}$ ratio (especially in the blue at $[\mathrm{OII}] \lambda 3727)$ of the $\mathrm{AAT} / 2 \mathrm{dF}$ spectra has allowed us to probe lower emission levels than in previous analyses. 


\subsection{Chandra ACIS-I Imaging}

Abell 3128 was observed on 5 May 2000 for a total of 19,513 seconds using the Chandra X-ray Observatory with the Advanced CCD Imaging Spectrometer (ACIS) in its default imaging mode (ACIS-I). The field center of the observation is 03:30:21.93 -52:31:51.5, i.e., approximately midway between the two X-ray peaks previously identified on ROSAT archival images. In ACIS-I imaging mode, the primary field is imaged onto four front-illuminated CCD chips (referred to as I0-I3), arranged in a $2 \times 2$ array so as to cover a field of $16^{\prime} \times 16^{\prime}$. The telescope is dithered (by $16^{\prime \prime}$ peak to peak) during the exposure, to provide exposure in the $(\sim 11$ ") gaps between the CCD chips. The results presented here are based on reprocessed data (revision 2), and the events are filtered to include events with flight grades $0,2,3,4$ and 6 and energy $<10 \mathrm{keV}$. For the imaging analysis the lower energy range was restricted to 0.5 to $10 \mathrm{keV}$. The background during this observation was free of flares or enhancements, so no data was rejected because of high background.

The imaging analysis was performed after correcting the data for vignetting and position dependent gain effects (primarily due to charge transfer inefficiency resulting from radiation damage) with the current calibration files in 5 energy bands, $0.5-1.5,1.5-2.5,2.5-4.0,4.0-6.0$, and 6.0 $-10.0 \mathrm{keV}$. These were then combined into a single broadband image from 0.5 to $10.0 \mathrm{keV}$.

Background determination for extended sources can be notoriously difficult when the source fills most of the field of view, which is the case for this observation (see fig 17). By examining the data in several bands and at various smoothing scales we found two regions that are free of cluster emission, one on the extreme NW corner of the I0 chip and another on the far SW corner of the I3 chip. These regions are used as the background for the imaging analysis and for the spectral analysis of the cluster emission.

\subsection{Radio Observations}

\subsubsection{MOST data}

The Molonglo Observatory Synthesis telescope (MOST) is an east-west synthesis array comprising two colinear cylindrical paraboloids each $12 \mathrm{~m}$ wide by $778 \mathrm{~m}$ long, separated by a $15 \mathrm{~m}$ gap (Robertson 1991). It operates at a frequency of $843 \mathrm{MHz}$ with a $3 \mathrm{MHz}$ bandwidth. For the observation of A3128 the synthesized FWHM beamwidth was $43^{\prime \prime}(\mathrm{RA}) \times 54^{\prime \prime}(\mathrm{Dec})$ and the field

of view was $70^{\prime}(\mathrm{RA}) \times 88^{\prime}(\mathrm{Dec})$. The data reduction used standard in-house software and the rms noise in the CLEANed image was $0.85 \mathrm{mJy} /$ beam. 


\subsubsection{ATCA data}

The Australia Telescope Compact Array (ATCA) is a 6-element east-west array with a maximum baseline of $6 \mathrm{~km}$. Simultaneous observations were made in two frequency bands, 1384 and $2496 \mathrm{MHz}$, each of bandwidth $128 \mathrm{MHz}$. Short observations were made with different antenna configurations in 1999 February, 2000 March and November. These data sets were co-added after calibration, giving a total integration time of 3.6 hours.

All data reduction was performed using the MIRIAD software package (Sault et al. 1995). The primary flux density calibrator was PKS B1934-638, and the phase calibrator PKS B0334-546. The resultant beamshapes were $15^{\prime \prime} \times 10^{\prime \prime}$ in $\mathrm{PA}-20^{\circ}$ at $1384 \mathrm{MHz}$ (natural weighting), and $5.2^{\prime \prime} \times 3.2^{\prime \prime}$ in $\mathrm{PA}-10^{\circ}$ at $2496 \mathrm{MHz}$ (uniform weighting).

Full details of the radio observations are given in Table 1.

\section{Spatial/Kinematic Substructure in A3128/A3125}

Kinematic signatures of groups and other structures within a cluster can be used, in principle, to assess whether clusters are indeed formed through a merger hierarchy. In this Section, we make an empirical examination of the spatial-kinematic distribution of the galaxies in A3128/A3125, to look for the presence of substructures on various scales. In what follows we begin with the largest scale features found in our galaxy database, and then work toward smaller scale groups, before turning in $\S 4$ to substructure in the ICM. A key challenge will be to assess the statistical reality of apparent groupings identified in the galaxy data.

An overall impression of the A3128/A3125 spatial-kinematic structure can be obtained from the Dec versus RA plot in Fig. 2. The RA and Dec axes are plotted in units of arcminutes from the center of A3128. We have plotted all galaxies from our sample within the redshift range of $9000<\mathrm{cz}<29000 \mathrm{~km} \mathrm{~s}^{-1}$, but with those galaxies in the narrower velocity range $16000<\mathrm{cz}$ $<19500 \mathrm{~km} \mathrm{~s}^{-1}$ plotted as filled squares. The locations of the two bright X-ray peaks, discussed in $\S 4$, are plotted as large six-pointed stars. There is a marked contrast between A3128, which exhibits a clear central galaxy concentration, and the less massive A3125, which has a dispersed appearance. The latter cluster is primarily located between $0^{\prime}$ and $-50^{\prime}$ in $\Delta \alpha$ and between $-80^{\prime}$ and $-25^{\prime}$ in $\Delta \delta$ in Fig. 2. On the basis of N-body simulations, Caldwell \& Rose (1997) argue that the dispersed nature of A3125 is due to the tidal effect of its recent passage through A3128 (i.e., we are now observing A3125 after a close passage through A3128). 


\subsection{Gaps in the Velocity Histogram Around A3128/A3125}

In Figs. 3 and 4 we plot position-velocity diagrams in both RA and Dec. ${ }^{2}$ In Fig. 3 the large velocity range from $5000 \mathrm{~km} \mathrm{~s}^{-1}$ to $70000 \mathrm{~km} \mathrm{~s}^{-1}$ is included, while in Fig. 4 we emphasize the more restricted velocity region between $9000 \mathrm{~km} \mathrm{~s}^{-1}$ and $29000 \mathrm{~km} \mathrm{~s}^{-1}$. The nonuniform distribution in velocity is readily apparent. Aside from the obvious A3128/A3125 cluster peak at $\sim 18000 \mathrm{~km} \mathrm{~s}^{-1}$, there are concentrations as well at $\sim 13000 \mathrm{~km} \mathrm{~s}^{-1}, \sim 23000 \mathrm{~km} \mathrm{~s}^{-1}$, and $\sim 33000 \mathrm{~km} \mathrm{~s}^{-1}$, i.e., at $-5000 \mathrm{~km} \mathrm{~s}^{-1},+5000 \mathrm{~km} \mathrm{~s}^{-1}$, and $+15000 \mathrm{~km} \mathrm{~s}^{-1}$ with respect to the the mean cluster velocity. Due to our interest in A3128/A3125, and its relation to the surrounding H-R Supercluster, in what follows we restrict our attention to the velocity peaks at $\sim 13000 \mathrm{~km} \mathrm{~s}^{-1}$ (or double peak at $\sim 12000 \mathrm{~km} \mathrm{~s}^{-1}$ and $\sim 14000 \mathrm{~km} \mathrm{~s}^{-1}$ ), $\sim 18000 \mathrm{~km} \mathrm{~s}^{-1}$, and $\sim 23000 \mathrm{~km} \mathrm{~s}^{-1}$ , which are better seen in Fig. 4.

To further emphasize the uneven distribution of redshifts in the direction of the A3128/A3125 system, we plot the histogram of velocities in Fig. 5. The most striking aspect of the histogram is the fact that while the velocity distribution drops off sharply, as expected, at $\sim 1500 \mathrm{~km} \mathrm{~s}^{-1}$ on both sides of the central cluster redshift at $\sim 17775 \mathrm{~km} \mathrm{~s}^{-1}$, the distribution increases again substantially at $\sim 4000 \mathrm{~km} \mathrm{~s}^{-1}$ on both high and low velocity sides, i.e., at $\sim 14000 \mathrm{~km} \mathrm{~s}^{-1}$ and $\sim 22000 \mathrm{~km} \mathrm{~s}^{-1}$. On one hand, the dip in the histogram, followed by a subsequent rise at both high and low velocity, is basically in accord with the expectation that a cluster will carve out a local velocity void around it, and that the velocity distribution then returns to the unperturbed Hubble flow beyond the cluster turnaround radius. On the other hand, the turnaround radius for a cluster with velocity dispersion of $1000 \mathrm{~km} \mathrm{~s}^{-1}$ is expected to be at $1500 \mathrm{~km} \mathrm{~s}^{-1}$ (Kaiser 1987), while the rebound in the A3128/A3125 velocity distribution is at $4000 \mathrm{~km} \mathrm{~s}^{-1}$. As will be discussed further in $\S 5$, we attribute the abnormally large gap in the Hubble flow that is inferred from the velocity histogram in Fig. 5 to the gravitational influence of the H-R Supercluster. The implication is that not only is there an abnormally large void in the Hubble flow produced by the H-R Supercluster, but that groups of galaxies falling into A3128/A3125 after detaching from the Hubble flow are accelerated to unusually high infall velocities, due primarily to the $H$ - $R$ Supercluster, and secondarily to the acceleration caused by A3128/A3125 itself.

\subsection{Identification of Galaxy Groups and Filaments}

A second interesting aspect of the position-velocity plots shown in Fig. 4 is the apparently non-uniform distribution of galaxies in certain areas of the plots, which indicate the presence of spatial-kinematic substructures in A3128/A3125. The apparent groupings are most noticeable on the high velocity side of the cluster. We have identified several groupings, selected either on the

\footnotetext{
${ }^{2}$ For greater ease of comparison between RA and Dec plots we have chosen not to display the data in the form of a traditional wedge diagram.
} 
basis of their clumping in RA-cz or Dec- $c z$ space. These candidate groups are identified in positionvelocity diagrams in Fig. 6, where they are plotted with different colors and symbols. We have identified two types of groups; (1) those which are fairly symmetrically distributed in position and velocity (such as the group, with magenta open circles, centered at Dec $=-60^{\prime}$ and cz $=18600$ $\mathrm{km} \mathrm{s}^{-1}$ ), and (2) those which appear to form filaments in position-velocity space (such as the group highlighted by red squares which stretch from $D e c=-10^{\prime}$ and $c z=19300 \mathrm{~km} \mathrm{~s}^{-1}$ to $\mathrm{Dec}=20^{\prime}$ and $\mathrm{cz}=20300 \mathrm{~km} \mathrm{~s}^{-1}$ ). The locations of these groupings in RA-Dec space are plotted in Fig. 7, where the same colored symbols of the groups identified in Fig. 6 are carried over into the RA-Dec plot. Specifically, we have selected two candidate groups (plotted as magenta and green colored annuli) and two candidate filaments (plotted as red and blue colored filled squares). The typical spatial dimension of these systems is $\sim 10-20^{\prime}$, or $\sim 1-2 \mathrm{Mpc}$ for our assumed value of $50 \mathrm{~km} \mathrm{sec}^{-1} \mathrm{Mpc}^{-1}$ for $\mathrm{H}_{\circ}$. The kinematic length of the filaments, however, at $\Delta \mathrm{cz} \sim 1000 \mathrm{~km} \mathrm{~s}^{-1}$, implies a spatial length in the Hubble flow of $\sim 20 \mathrm{Mpc}$, an implication which is discussed in $\S 6.3$.

Are the groups and filaments identified in Fig. 6 truly physical groups or just chance associations selected by eye? Unfortunately, testing the statistical reality of such groupings, given that our analysis is clearly ex post facto (we first select apparent groupings in the plots, and then statistically analyze whether they are indeed real), is notoriously suspect. With any large sample of data points, it is not difficult to find a suggestive grouping in the data, for which an ex post facto analysis produces a high degree of confidence that the grouping is not random. To make a more realistic assessment of the reality of the group, one needs to determine what is the likelihood in a data sample (of a particular overall size) of finding at least one grouping that is as large as the one selected. Such an analysis in principle could be performed in the following manner. One could simulate a large number of clusters similar to A3128/A3125 by randomly selecting galaxies from the observed redshift distribution of Fig. 5 and then distributing them at random in RA and Dec over the region covered by our data sample. One could then assess how frequently groups as large and as concentrated as the ones selected in Fig. 6 show up in these random simulations. It should be clear to the reader, however, that the distribution of galaxies in, especially, the redshift region $18500 \mathrm{~km} \mathrm{~s}^{-1}<22000 \mathrm{~km} \mathrm{~s}^{-1}$ is indeed non-random and will indicate clumping at a high statistical level. The problem is that by setting up a baseline of complete randomness in RA and Dec, we are in effect setting up a statistical strawman to knock down.

We use, instead, the following statistical approach. In principle, the RA versus $c z$ plot is statistically independent from the Dec versus $c z$ plot. Therefore, if we select a candidate group or filament that is localized in both position and velocity in the RA versus $c z$ plot, but that is actually just a false statistical group, the data points should appear randomly spread in Dec in the corresponding Dec versus $c z$ plot. An example of such behavior is shown in Figs. 8 and Fig. 9, where an apparent tight group of points localized between $-30^{\prime}$ and $-22^{\prime}$ in $\alpha$ and between 17500 $\mathrm{km} \mathrm{s}^{-1}$ and $18300 \mathrm{~km} \mathrm{~s}^{-1}$ in redshift is spread widely in $\delta$. In contrast, the groups and filaments selected in either RA or Dec in Fig. 6 are more localized in the corresponding coordinate than the pseudo group shown in Fig. 8. The RA versus Dec plot of this pseudo group in Fig. 9 confirms 
the non-association of the galaxies.

To provide a quantitative footing to the above approach, in each case that we find a candidate group localized in RA (or Dec) and $c z$, we create a control sample containing all data points within the identifed $c z$ limits for that group, excluding the group members themselves. We then apply three statistical measures to assess the reality of the candidate group or filament in three dimensional $\alpha-\delta$-cz space. First, we apply the Kolmogorov-Smirnoff (K-S) two-sample test to the group versus control sample in the following manner. If the candidate group has been defined in $\alpha$ and $c z$, we apply to the K-S test to the $\delta$ values, to assess the likelihood that the $\delta$ values of the group and control samples come from the same parent population. If the group has been selected in $\delta$, then we apply the K-S test to the $\alpha$ values. Second, we make a linear least squares analysis for a correlation between RA and $c z$, and also test for a correlation between Dec and $c z$. Third, we measure the rms dispersion in both the $\alpha$ and $\delta$ coordinates for each group, and for each control sample. The results of the statistical tests are given in Table 3. In column (1) the group identification is given, where G1 and G2 are the two candidate groups (the green and magenta colored annuli respectively); F1 and F2 are the two candidate filaments (the red and blue colored squares respectively); $\mathrm{C} 1$ is the control group from Fig. 8. In column (2) is given the number of group members, while in columns (3), (4), and (5) are given the limits in $c z, \alpha$, and $\delta$ for each group respectively. In column (6) are listed the results of applying the Kolmogorov-Smirnov two-sample test to each group versus its respective control sample; the number given is the likelihood for the hypothesis that the group and its control are drawn from the same parent sample. In columns (7) and (8) are listed the results the linear least squares regression analysis for RA versus $c z$ and Dec versus $c z$ respectively. The numbers represent the probability that the variables are uncorrelated. Finally, in columns (9) and (10) are given the rms deviations in the RA and Dec coordinates for the groups. Immediately below each group in Table 3 is given the data for its specific control sample, for which selection is made in $c z$ only.

The two groups, G1 and G2, stand out clearly in having low $\sigma_{\alpha}$ and $\sigma_{\delta}$ compared to their control samples. In addition, the K-S test indicates a very high confidence that they are different from their control samples. On the other hand, there is no indication of correlation in $\alpha-c z$ or $\delta-c z$, which is expected since neither group appears to have a filamentary elongation. In contrast, the control group C1, while defined to have a small spread in $\alpha$, shows a large spread in $\delta$. Surprisingly, the K-S test rejects at a $97.5 \%$ likelihood the hypothesis that the C1 group and its own control group are selected from the same sample. However, the $\mathrm{C} 1$ group was defined to have a low mean RA, which places it away from the main concentration of A3128. Therefore, the distribution of Dec values in this pseudo group do not reflect that of the main A3128 cluster. The control group for $\mathrm{C} 1$ is defined in $c z$ only, hence the distribution in Dec shows the expected concentration around A3128. As a consequence, the K-S test indicates that the samples are different. Thus although the Dec values in the C1 "group" are clearly spread quite randomly (see Figs. 8 and 9), the K-S test does not actually test that fact.

The case of the two filaments, F1 and F2, is less clear. The principal evidence that F1 and 
F2 are real structures lies in the correlation statistics between $\alpha$ and $c z$ and between $\delta$ and $c z$. For F1, the probability of a correlation is high in both coordinates, while for F2 the probability is only significant in $\delta$ versus $c z$. A high correlation is also found in $\alpha$ versus $c z$ for the two control samples. This is due to the fact that the control samples for both F1 and F2 contain parts of both filaments. Since F1 and F2 are widely separated in $\delta$ and also have a mean offset in $c z$, a high correlation coefficient is found. This result reinforces the above example regarding the K-S result for the $\mathrm{C} 1$ control sample, namely, that blindly applied statistical tests can be misleading. Overall, we consider the case for the reality of F1 to be strong, given the high correlation probability in both RA and Dec. The statistical case for F2, on the other hand, rests entirely on the correlation found in $\delta$ versus $c z$, and thus needs to be taken with caution. We also note that G1 joins onto F1, as does G2 onto F2. Thus we have perhaps artificially separated a group component from a filament component.

In addition to the G1, G2, F1, and F2 groups shown in Figs. 6 and 7, we have selected five additional small groups, each with less than 10 members, which are plotted in Figs. 10 and 11. For these latter groups, the only statistical test that we have applied is to measure the dispersions in RA and Dec, as well as for their corresponding control samples. The results of that analysis is summarized in Table 4. In all cases, the small rms dispersion in one coordinate is matched in the other coordinate, particularly when a single discordant galaxy is removed. In contrast, the rms dispersion in the corresponding control sample is always substantially larger, except in the case of group G6, for which the control sample of only 4 galaxies appears to constitute a small group of its own.

A final comment about the statistical reality of groups concerns the linear velocity gradient in the A3125 cluster reported in Caldwell \& Rose (1997), on the basis of 19 galaxies, which is typical of the numbers in G1, G2, F1, and F2. That linear velocity gradient is no longer evident in A3125, now that a much larger sample of galaxies has been observed. In particular, the presence of G2 and F2 in the SW, which due to the smaller area sampled by Caldwell \& Rose (1997) was missed by them, has swamped the original gradient. It appears likely that Caldwell \& Rose (1997) detected a gradient produced by sampling primarily from two galaxy clumps with different mean redshift. In short, when small numbers of galaxies are involved, it is difficult to distinguish between a real filamentary gradient and an apparent gradient produced by the offset properties of two groups.

\subsection{Distribution of Foreground and Background Galaxies}

Turning now to the concentrations of galaxies in the foreground and background of A3128/A3125, we have subdivided the foreground and background into various velocity slices and plotted them in position-velocity space in Fig. 4, with different colors referring to the different velocity slices, and annuli and filled squares representing foreground and background galaxies respectively. The positions of the color-coded galaxies are repeated in an $\alpha-\delta$ plot in Fig. 12. An intriguing aspect of Figs. 4 and 12 is the nonuniform distribution of foreground versus background galaxies. There is 
an overall tendency for the foreground galaxies (colored unfilled circles) to populate the NE region, i.e., to concentrate around A3128, while the background galaxies (colored squares) lie primarily in the southern portion of the plot. Caution must again be exercised in making a statistical argument about this offset between foreground and background, since the background galaxies, in particular, appear to be somewhat concentrated into groups. The existence of background group G7 in the SE has already been pointed out in Figs. 10 and 11. As a result, the foreground and background galaxy distributions might well result from just a few independent groups. Thus statistical tests for differences between the distribution of foreground versus background galaxies, based on assuming all galaxies to be independent points, will not accurately reflect that situation.

To further illustrate the differences in spatial distribution between foreground and background, we plot the galaxy redshifts versus their radial distance from the center of A3128 in Fig. 13. The large $\left(\sim 4000 \mathrm{~km} \mathrm{~s}^{-1}\right)$ gap in $c z$ between cluster and both foreground and background is again clearly evident. In addition, while the foreground galaxies tend to have radial distances in the range $0^{\prime}$ to $50^{\prime}$, the background galaxies largely avoid the central $20^{\prime}$.

\subsection{Statistics of Emission Line Galaxies}

As previously mentioned, due to problems with the continuum levels near $[\mathrm{OII}] \lambda 3727$ for the fainter galaxies, it was not possible to obtain reliable emission line equivalent widths. However, we are able to search for [OII] $\lambda 3727$ emission in the galaxies to typically $0.5 \AA$ levels in equivalent width. As a result we have compiled statistics of the percentage of galaxies with observed emission, as a function of their environment. Here we have restricted the discussion to only those galaxies with $\mathrm{AAT} / 2 \mathrm{dF}$ spectra, since those spectra provide the most homogeneous set to work from.

We find a surprisingly high percentage of galaxies in the main A3128 cluster have detectable [OII] $\lambda 3727$ emission. Specifically, 41 of 85 galaxies, or 48\%, with radial velocity between 16000 $\mathrm{km} \mathrm{s}^{-1}$ and $19200 \mathrm{~km} \mathrm{~s}^{-1}$, and with Declination North of -53:01:30 (the center of A3128 is at $\delta=-52: 31: 30$, J2000) have emission. This is an unusually high level of emission, given that most surveys of emission in rich clusters report emission detection rates below 15\% (Biviano et al. 1997). In contrast, Biviano et al. (1997) report emission line detections for only 30 out of 152 galaxies in their data on A3128, i.e., an $\sim 20 \%$ detection rate. Thus the high fraction of galaxies found with emission lines is partly the result of the high $\mathrm{S} / \mathrm{N}$ ratio achieved for the $2 \mathrm{dF}$ spectra, but also partly due to the fact that A3128 has an unusually high level of emission, since the $20 \%$ detection rate reported by Biviano et al. (1997) is at the high end for the ENACS cluster sample.

While the main cluster emission-line galaxy fraction is high, the detection rate in our $2 \mathrm{dF}$ data of galaxies in foreground and background flows is substantially higher. A total of 38 out of 54 galaxies, i.e., $70 \%$, of the galaxies in the background within the velocity range $22100 \mathrm{~km} \mathrm{~s}^{-1}$ to $28500 \mathrm{~km} \mathrm{~s}^{-1}$ have detected [OII] $\lambda 3727$ emission, while 15 out of 16 , or $94 \%$, of the foreground

galaxies in the velocity range $10000 \mathrm{~km} \mathrm{~s}^{-1}$ to $15000 \mathrm{~km} \mathrm{~s}^{-1}$ are also detected. The statistics 
of the group and filament galaxies is that 32 out of 48 of them,i.e., $67 \%$, have detected emission. Thus, while the emission line statistics of the main A3128 cluster are remarkably high, indicating perhaps an overall dynamical youth of the cluster, the emission levels are still lower than in the immediate field and in infalling groups. Finally, of the 19 galaxies in the background redshift peak at $\mathrm{cz} \sim 33000 \mathrm{~km} \mathrm{~s}^{-1}$, 16 of them (i.e., 84\%) have detected [OII] $\lambda 3727$ emission, hence indicative of a field population. Note that the detection of $[\mathrm{OII}] \lambda 3727$ emission alone does not allow us to distinguish between emission due to star formation and that caused by an active galactic nucleus.

To conclude at this point, data on the distribution of galaxies in the field of A3128/A3125 indicates that spatial/kinematic structures are present on a variety of scales. The most evident structure is the $\sim 4000 \mathrm{~km} \mathrm{~s}^{-1}$ underpopulated velocity region on both sides of the cluster velocity. Much smaller structures are evident as well, ranging from groups of 4 galaxies to groups with a couple of dozen members, and even the foreground and background galaxies at $4000 \mathrm{~km} \mathrm{~s}^{-1}$ on either side of the A3128 systemic velocity are nonuniformly distributed. The majority of galaxies in the underpopulated velocity regions appear to be members of groups and/or filaments. However, the statistical reality of these groups (and particularly of the filaments) is difficult to establish. Most of the groups identified have high velocities with respect to the A3128/A3125 double cluster. Such high velocities of these infalling and merging substructures should in principle lead to unusual transient physical conditions in the hot intracluster medium, a subject which we turn to in the next section, where we consider the Chandra X-ray images of A3128.

\section{X-ray Substructure in the Intracluster Medium}

Aside from spatial-kinematic substructures evident in the galaxy distribution, substructure in the hot ICM also provides clues to past and ongoing cluster merger events. The ICM has the particular advantage of not suffering from the discreteness problem that afflicts the statistical selection of small galaxy groups. Consequently, we now turn to the Chandra ACIS-I observations of the central region of A3128.

The X-ray morphology of the central $16^{\prime} \times 16^{\prime}$ of A3128 is illustrated in Figs. 14 and 15, where the $20 \mathrm{ks}$ Chandra image is displayed at two different contrast levels, for photons in the energy range $0.5 \mathrm{keV}$ to $10 \mathrm{keV}$. In all cases the original image has been $4 \times 4$ binned into 2 " x 2 " pixels, and then further smoothed with a gaussian of $\sigma=4$ pixels. The most evident feature of the cluster X-ray morphology is the presence of two bright X-ray peaks, aligned in the NE-SW axis, and

separated by $12^{\prime}$, or $1.2 \mathrm{~h}_{50}^{-1} \mathrm{Mpc}$. These two peaks have been marked previously on figures such as Fig. 2. We hereafter refer to these two components as the NE and SW peaks. Also evident is a lower surface brightness component, located between the two bright peaks. This third component to the X-ray flux has a center that, to within the uncertainties, is indistinguishable from the center of the galaxy distribution in A3128. To further assess the morphology of the X-ray emission, we present in Fig. 16 an adaptively smoothed version of the Chandra image. Specifically, a maximum smoothing of $\sigma=5$ pixels was applied. Furthermore, to illustrate the connection between the X-ray 
emission and the galaxy distribution, we have plotted the Chandra X-ray contours on top of a grayscale representation of the optical $\mathrm{B}_{J}$ image in Fig. 17. It is evident that while the SW X-ray peak is coincident with a bright galaxy, and with an apparent compact galaxy group, there is no such general coincidence between the NE X-ray peak and the galaxy distribution, a point that we return to in $\S 6$.

A second noteworthy feature of the X-ray morphology is the fact that the two bright peaks have small core radii and are offset from the lower surface brightness emission on which they are superimposed, as can be seen qualitatively in, e.g., Fig. 16. To quantify the small core radii we have plotted in Fig. 18 the azimuthally averaged radial surface brightness profiles of the NE and SW X-ray components, plotted both in terms of linear radial distance and logarithmic radial distance. Also plotted are the best $\beta$ model fits to the NE and SW components, following the prescription of Cavaliere \& Fusco-Fumiano (1978), i.e., we fit the surface brightness distribution according to

$$
\Sigma(R)=\Sigma(0)\left[1+(R / a)^{2}\right]^{-3 \beta+1 / 2},
$$

where $a$ is the core radius of the fit. We obtain a core radius of $26 \mathrm{~h}_{50}^{-1} \mathrm{kpc}$ and $40 \mathrm{~h}_{50}^{-1} \mathrm{kpc}$ for the $\mathrm{NE}$ and SW components respectively. As will be further discussed in $\S 5$, these values are at the low end of the large range in core radii observed in clusters of galaxies (e.g., Jones \& Forman 1984; Peres et al. 1998; Mohr, Mathiesen, \& Evrard 1999), where a typical cluster has a core radius of $\sim 250 \mathrm{kpc}$. In addition, the $\beta$ values of $\sim 0.3$ derived from the fits are low compared to typical values for clusters of $\sim 0.7$.

To better illustrate the fact that the bright NE and SW cores are offset from their underlying lower surface brightness emission, we have fit elliptical isophotes to the NE and SW components at various surface brightness levels, using the 'ellipse' routine in the STSDAS 'isophote' package. The fits return the X-Y positions of the center of the fitted ellipse at each contour level, and the results have been plotted in Fig. 19. The X (RA) and Y (Dec) centers change by 55 pixels (110") and 20 pixels $\left(40^{\prime \prime}\right)$ respectively in the NE peak as one moves from the central peak out to a maximum of 200 " in the semi-major axis of the azimuthally averaged radial profile. In the SW peak the X and $\mathrm{Y}$ offsets are 25 pixels and 12 pixels respectively. Thus the effect is considerably more pronounced in the NE component, but clearly is present in the SW component as well. Moreover, while the shift in isophote center is fairly linear with semi-major axis, in the case of the NE component, the shift is very small within the central 20 pixels $\left(40^{\prime \prime}\right)$, then ramps up at larger radii. Note that the displacement of the core emission in both peaks relative to the lower surface brightness emission underlying them is in the sense that the emission falls off sharply on the side pointing away from the cluster and trails off more slowly in the cluster center direction.

A final morphological feature of interest is the resolved inner structure of the NE component, seen in Figs. 14 and 16. The most striking feature of the NE emission peak is its strong elongation along the axis connecting the NE and SW components. This elongation factor is substantial, as can be seen from the ellipticity and position angle profiles plotted in Fig. 20. While the mean ellipticity for the fainter contours is $\sim 0.4$, it rises to 0.65 in the inner 5 pixels $\left(17 \mathrm{~h}_{50}^{-1} \mathrm{kpc}\right)$ in 
radius. Furthermore, there is a substantial twist in the position angle of the fitted ellipse, from $60^{\circ}$ $\mathrm{E}$ of $\mathrm{N}$ in the inner $10^{\prime \prime}$ to $70^{\circ} \mathrm{E}$ of $\mathrm{N}$ at a semi-major axis of $50^{\prime \prime}$. In short, transient morphological features are present in the X-ray emission from the ICM of A3128 on a variety of scales, from less than $20 \mathrm{~h}_{50}^{-1} \mathrm{kpc}$ in the core of the NE component to the $\sim 1 \mathrm{Mpc}$ projected separation between the NE and SW components.

Another key diagnostic of the physical conditions in the ICM of A3128 is the temperature structure of the X-ray emitting gas. We have derived temperatures from the observed X-ray emission by using XSPEC (Arnaud 1996) to fit a MekaL (Mewe et al. 1985, 1986; Liedahl, Osterhedl, \& Goldstein 1995) thin plasma model with foreground absorption to the spectral data. The abundances are given relative to the solar values (Anders \& Grevesse 1989). In these fits the HI absorption, temperature, and abundance are allowed to vary, and we restrict our analysis to the $0.5-10 \mathrm{keV}$ band. The NE and SW components have global temperatures of $3.9 \mathrm{keV}$ and $3.6 \mathrm{keV}$ respectively, while the third, lower surface brightness, component has a temperature of $\sim 4 \mathrm{keV}$. Moreover, while the SW component appears to be isothermal, the data on the NE component suggests that the temperature is higher in the inner $45^{\prime \prime}$ in radius. The data on temperature and metal abundance, along with their $90 \%$ confidence limits, are summarized in Table 5. While the uncertainties are substantial, the data are suggestive of a temperature increase in the core of the NE component, and are in any case inconsistent with a substantial temperature drop in either the NE or SW component. Thus there is no indication of a cooling flow in either peak.

From the $\beta$ model fit to the radial surface brightness in the NE and SW components, and assuming the temperatures from the "global" fits to each component, we have calculated an estimated "virial" mass enclosed within a radius of $0.3 \mathrm{~h}_{50}^{-1} \mathrm{Mpc}$ for both components, by assuming hydrostatic equilibrium. For the NE and SW components we then derive a virial mass of $1.6 \mathrm{x}$ $10^{14} \mathrm{M}_{\odot}$ and $1.5 \times 10^{14} \mathrm{M}_{\odot}$, respectively. On the other hand, the gas mass in the NE and SW components (inside of $3^{\prime}$ or $0.3 \mathrm{~h}_{50}^{-1} \mathrm{Mpc}$ in radius) derived from the central electron densities of $1.1 \times 10^{-2} \mathrm{~cm}^{-3}$ and $2.4 \times 10^{-2} \mathrm{~cm}^{-3}$ is $6.5 \times 10^{12} \mathrm{M}_{\odot}$ and $7.2 \times 10^{12} \mathrm{M}_{\odot}$, respectively, for the $\mathrm{NE}$ and SW components (the X-ray luminosities of the two components are $5.4 \times 10^{43} \mathrm{erg} / \mathrm{sec}$ and $3.4 \times 10^{43} \mathrm{erg} / \mathrm{sec}$ in the $0.5-10 \mathrm{keV}$ band). Thus the implied gas-mass to virial-mass ratios are $\sim 4-5 \%$ in the two components, which is very low compared to more typical values of $\sim 20-30 \%$ usually found in rich clusters (e.g., Mohr, Mathiesen \& Evrard 1999). Seen another way, for the observed X-ray emitting gas masses, the virialized velocity dispersion in the galaxies for the NE and SW components should be $437 \mathrm{~km} \mathrm{~s}^{-1}$ and $425 \mathrm{~km} \mathrm{~s}^{-1}$ respectively. While the observed velocity dispersion of the galaxies cannot be readily separated into NE and SW components, and while in fact the presence of so much substructure in A3128 makes it difficult to define a global cluster velocity dispersion, it is clear in any case that the velocity dispersion of the galaxies in the center of A3128 is far in excess of $430 \mathrm{~km} \mathrm{~s}^{-1}$. Thus the discrepant values extracted from a dynamical analysis indicates that the X-ray emitting gas is not virialized and therefore must be well removed from hydrostatic equilibrium.

The third, diffuse emission component, seen between the two X-ray peaks (Fig. 15 and Fig. 
$16)$ is noteworthy in that its extent is approximately that of a normal cluster ( $\sim 3 \mathrm{Mpc})$, it is hot $(\mathrm{kT} \sim 4 \mathrm{keV})$, and is also enriched with an abundance of $\sim 0.6$ solar. The fact that the diffuse gas is enriched implies that it is or was bound to a system with stars which can supply the metals. Since there are two peaks with similar properties, determining which one might be associated with the diffuse emission component must be based on indirect evidence. From the global abundances in Table 5 we see that the NE component has a very low global abundance, $\sim 0.13$ solar, much lower than the best fit abundance of the diffuse component, which on the other hand is consistent with the abundance of the SW clump. So, while we cannot rule out that the diffuse component is associated with the NE component, it seems more likely to be associated with the enriched SW component. Thus the third, diffuse, component appears unlikely to have a common origin with the NE component.

Finally, we have obtained a direct estimate of the redshift of the X-ray gas in the NE, SW, and diffuse components using the X-ray emission lines present in the ACIS-I spectra. For the SW component, our best fit model, in which we allow the redshift to be a free parameter, produces a best fit redshift of $\mathrm{z}=0.065$, with $90 \%$ confidence limits between 0.050 and 0.078 . For the diffuse emission component, the best fit redshift is $\mathrm{z}=0.059$, with a $90 \%$ confidence range of 0.047 to 0.072 . Due to the lower counts and lower metal abundance in the NE component, the redshift of that gas is less well constrained. The best fit redshift is 0.10 , with a $90 \%$ confidence range of 0.037 to 0.23 . Thus we can conclude that the gas in the SW and diffuse components are unambiguously associated with A3128, while the redshift found for the NE component is consistent with A3128, but the association is not conclusive.

\section{Radio Sources in A3128}

Further insight into the events transpiring in A3128/A3125 can be gained from examination of radio images of the double cluster, since radio sources can be reenergized by ICM shocks produced in mergers (Ensslin et al. 1998; Roettiger, Burns, \& Stone 1999; Govoni et al. 2001). Furthermore, the morphology of tailed sources can be useful in assessing the relative direction of motion of the source with respect to any gas dynamical movement of the local ICM. We begin by displaying in Fig. 21 the MOST $843 \mathrm{MHz}$ radio contours superposed on the Chandra ACIS-I X-ray image. Four of the radio sources have X-ray counterparts, three of which appear to be cluster members, as revealed by the overlaid radio-optical plot in Fig. 22.

We will focus here on the two radio sources aligned with the NE and SW X-ray peaks. The weak unresolved source coincident with the SW X-ray core is identified with ENACS\#75, one of the brightest galaxies in the cluster. On the other hand, the radio source at the NE peak is both stronger and more remarkable. It appears to consist of a bright point source and a much fainter arclike feature, displaced to the NE of the bright source. At the higher resolution of the ATCA $20 \mathrm{~cm}$ and $13 \mathrm{~cm}$ images, however, the bright radio source resolves into an elongated north-south double, as shown in Fig. 24, where the ATCA $20-\mathrm{cm}$ and 13-cm contours are superposed on a grayscale 
rendition of the CTIO 0.9-m $\mathrm{R}$ bandpass image. This figure shows that the radio source is identified with a faint galaxy that is undoubtedly background to A3128. The spatial alignment of optical galaxy, MOST and ATCA radio contours, and X-ray contours is illustrated in Fig. 25 CTIO 0.9-m $\mathrm{B}$ and $\mathrm{R}$ images of the region in Fig. 23 show that the faint galaxy has a concentrated red nucleus. In the deeper R-band image a somewhat irregular low surface brightness fuzz surrounds the bright nucleus, while in the B image, a long curved structure is seen primarily to the southwest. A hint of this curved structure is seen in the $\mathrm{R}$ image as well, but it clearly has a very blue color relative to the nucleus of the galaxy. In the unlikely circumstance that the galaxy is a member of A3128, it would be a low-luminosity dwarf, and hence extremely unlikely to harbor a compact luminous double radio source. Thus we infer that the bright double is in fact a superposed background source that has no physical relation to $\mathrm{A} 3128^{3}$. On the other hand, the faint extended radio arc seen in the MOST image (but not in the higher resolution ATCA $20 \mathrm{~cm}$ image), is more intriguing. It is situated slightly to the NE of the core emission in the NE X-ray component, about where one might expect a bow shock to be present if the NE component were plowing supersonically through A3128. The fact that the radio arc is displaced from the bright background source suggests that it truly is a structure associated with A3128, but deeper radio imaging is needed to better assess that conjecture.

\section{Discussion}

The single most compelling feature of the combined $2 \mathrm{dF}$ spectroscopy and Chandra X-ray imaging is the wide variety of structures observed in both the spatial/kinematic distribution of the galaxies and the hot ICM. On the largest scales there are peaks in the velocity distribution, separated by $\sim 4000 \mathrm{~km} \mathrm{~s}^{-1}$, i.e., over inferred distances of $\sim 80 \mathrm{~h}_{50}^{-1} \mathrm{Mpc}$, while on the smallest scales the X-ray image of A3128 shows transient structures $\lesssim 20 \mathrm{~h}_{50}^{-1} \mathrm{kpc}$ in size. In this section we attempt to draw a coherent picture encompassing the structures found on all scales, and to establish a link between galaxy substructure and the disturbed state of the ICM. In what follows, we begin with the largest structures, to establish the overall potential field in which the smaller substructures are embedded, and then work toward the smaller scales.

\section{1. $4000 \mathrm{~km} \mathrm{~s}^{-1}$ Velocity Gaps and the Horologium-Reticulum Supercluster}

As has been pointed out before, the velocity histogram in Fig. 5 indicates that on either side of the huge velocity peak at $\sim 17750 \mathrm{~km} \mathrm{~s}^{-1}$, representing the velocity center of A3128/A3125, there is a large "gap" of $\sim 4000 \mathrm{~km} \mathrm{~s}^{-1}$, in which the galaxy density is low, picking up again at $\sim 14000$

\footnotetext{
${ }^{3}$ If we assume a redshift $z \sim 0.3$, consistent with the galaxy's $\mathrm{R}$ magnitude (see Section 6.4.6), the integrated radio power at $1.4 \mathrm{GHz}$ is $\sim 2 \times 10^{25} \mathrm{~W} \mathrm{~Hz}^{-1}$, making it a powerful source, near the boundary between Fanaroff-Riley classes FRI and FRII
} 
$\mathrm{km} \mathrm{s}^{-1}$ on the low velocity side, and at $\sim 22000 \mathrm{~km} \mathrm{~s}^{-1}$ on the high velocity side. It is natural to assume that the increase in galaxy density at $\sim 14000 \mathrm{~km} \mathrm{~s}^{-1}$ and $\sim 22000 \mathrm{~km} \mathrm{~s}^{-1}$ represents the resumption of the Hubble flow on either side of the double cluster. While this assumption might be compatible with the galaxy distribution in redshift on the low velocity side, the distribution at high redshift is puzzling. There is a strong peak in redshift at $\sim 23000 \mathrm{~km} \mathrm{~s}^{-1}$, but then the histogram falls off precipitously to higher redshift, and between $\sim 25000 \mathrm{~km} \mathrm{~s}^{-1}$ and $\sim 32000$ there are very few galaxies found. The histogram then rises to another peak between $\sim 32000$ and $\sim 35000$ $\mathrm{km} \mathrm{s}^{-1}$, before dropping off once again to higher redshift. We note that the spatial distribution of galaxies in the redshift peak at $\sim 33000 \mathrm{~km} \mathrm{~s}^{-1}$ show no indication of being concentrated into a background cluster. Moreover, as mentioned before the fraction of emission line galaxies in the $\sim 33000 \mathrm{~km} \mathrm{~s}^{-1}$ peak is $84 \%$, indicative of a field, rather than a cluster, population. The behavior of the redshift histogram over the full range out to $\mathrm{cz}=65000 \mathrm{~km} \mathrm{~s}^{-1}$ is shown in Fig. 26 .

The velocity peaks at $\sim 14000 \mathrm{~km} \mathrm{~s}^{-1}$ and $\sim 23000 \mathrm{~km} \mathrm{~s}^{-1}$ are quite narrow, certainly less than $5000 \mathrm{~km} \mathrm{~s}^{-1}$ in full width, indicating that they might represent galaxy clusters at these redshifts. However, the galaxies in the peaks are distributed over the full $2^{\circ}$ field of the $2 \mathrm{dF}$ observations, and show no concentration into clusters. Rather, as was discussed in $\S 3$, there is some evidence to indicate an overall NE-SW asymmetry between the distribution of low-velocity peak versus highvelocity peak galaxies, which may result from the clumping of these galaxies into groups. Thus, while it is perhaps problematic to associate the peaks at $\sim 14000 \mathrm{~km} \mathrm{~s}^{-1}$ and $\sim 23000 \mathrm{~km} \mathrm{~s}^{-1}$ with the resumption of normal Hubble flow outside of A3128/A3125, we conclude here that the low and high velocity peaks on either side of the double cluster are the working "surfaces" from which new galaxies are being recruited into the A3128/A3125 system. The large, $\sim 4000 \mathrm{~km} \mathrm{~s}^{-1}$, velocity gap between these surfaces and the central cluster velocity is far in excess of that expected from a cluster of normal mass, and can only be attributed to a much greater massive concentration (Kaiser 1987). As has been pointed out previously, A3128/A3125 is embedded in the HorolgiumReticulum Supercluster, which extends over an area at least $7^{\circ}$ in extent (Lucey et al. 1983). The H-R Supercluster and Shapley Supercluster are the two largest known mass concentrations in the local universe, i.e., out to $300 \mathrm{~h}_{50}^{-1} \mathrm{Mpc}$ (Zucca et al. 1993; Einasto et al. 1994; Hudson et al. 1999). In fact, Zucca et al. (1993) and Einasto et al. (1994) consider the H-R Supercluster to encompass 18 and 32 clusters respectively. Thus we associate the largest structures in our data, i.e., the $\sim 4000$ $\mathrm{km} \mathrm{s}^{-1}$ gaps to the low and high velocity of A3128/A3125, with the huge gravitational potential well of the H-R Supercluster. The most important implication of this conclusion is that groups of galaxies which are detached fom the working "surfaces" are accelerated to unusually high infall velocity on their way toward A3128/A3125, due to the acceleration produced by the deep potential well of the H-R Supercluster. Specifically, the infall velocities will be supersonic with respect to the local sound speed in the A3128/A3125 complex, which, at an electron temperature of $4 \mathrm{keV}$, corresponds to $\sim 1000 \mathrm{~km} \mathrm{~s}^{-1}$.

To put the redshift histogram of the A3128/A3125 region into context, there are strong similarities with the observed distribution in redshift of galaxies in the Shapley Supercluster found by, 
e.g., Quintana et al. (2000) and Drinkwater et al. (1999). In the case of the Shapley Supercluster, the area surveyed is very extensive, so that the complexity of the large gaps in redshift, similar in size to the $4000 \mathrm{~km} \mathrm{~s}^{-1}$ gaps found by us in the H-R Supercluster, is readily seen in, e.g., Figs. 6 and 7 of Quintana et al. (2000). In contrast, where a large body of redshift data has been collected in the vicinity of a cluster that is not associated with a mass concentration on the scale of the H-R Supercluster, such as the Coma cluster (Geller, Diaferio, \& Kurtz 1999), no large gaps are evident in the distribution of velocities. Thus the association of the velocity gaps in A3128/A3125 with the potential well of the H-R Supercluster is further supported by comparison to other cases.

\subsection{The A3128/A3125 Double Cluster System}

The next clearly defined scale below that of the H-R Supercluster is the double nature of the A3128/A3125 cluster system. Much of this has been discussed in Caldwell \& Rose (1997), hence we repeat only a few key points. The projected separation between the center of A3128 and the very poorly-defined center of A3125 is on the order of $1^{\circ}$, which corresponds to $\sim 6 \mathrm{Mpc}$. Surprisingly, A3125 and A3128 have nearly the same mean cluster velocity, i.e., there is little relative motion between them along the line of sight. Thus one cannot reconcile the large distance between the two clusters with the small velocity separation (without appealing to a highly preferred viewing angle), unless A3125 is currently near turnaround after its passage by A3128. It is difficult to define reliable global velocity dispersions for A3125 and for A3128, given the large number of groups and filaments present whose inclusion or exclusion greatly affects the derived $\sigma$ (and given the fact that A3125 is not a clearly defined cluster). However, it is certainly clear that the velocity dispersion of A3125 is lower than that of A3128, which is in accord with the fact that A3128 contains 4 times more members than A3125. In addition, while A3128 has a well-defined central concentration in the galaxy distribution, A3125 presents a dispersed appearance. Based on N-body simulations, Caldwell \& Rose (1997) attribute the scattered appearance of A3125 to a tidal passage of the latter past A3128. In addition, while the $20 \mathrm{ks}$ Chandra exposure of A3128 reveals extensive emission from a hot ICM, the $10 \mathrm{ks}$ exposure of A3125 reveals no extended emission. What appears to be extended emission in an archival ROSAT $4 \mathrm{ks}$ image is actually the merging of badly degraded point sources, due to the large off-axis location of A3125 in the FOV of the ROSAT data. If there were any structures in A3125 as bright as the NE and SW components seen in A3128, they would easily be detected in the $10 \mathrm{ks}$ Chandra image of A3125. Consequently, the available evidence is consistent with A3125 being a relatively small cluster that has experienced a damaging encounter with A3128.

\subsection{Intermediate-Sized Groups and Infall into A3128}

We turn now to the intermediate-sized groups (10-30 observed members) and filaments. Natu-

rally, such groups are very difficult to locate in the crowded regions of A3128 and A3125, if they do 
exist there, but are readily seen in the low density $4000 \mathrm{~km} \mathrm{~s}^{-1}$ 'gaps' between the clusters and the higher density regions outside the H-R Supercluster. While identification of galaxies with groups and filaments can be problematic on statistical grounds, especially in regard to filaments, nearly all galaxies present in the high-velocity 'gap' region can be identified with intermediate-sized and small groups or filaments. The two filaments are particularly interesting. While their spatial extent of $\sim 20^{\prime}$ corresponds to a length of $2 \mathrm{~h}_{50}^{-1} \mathrm{Mpc}$, their velocity distension of $\sim 1000 \mathrm{~km} \mathrm{~s}^{-1}$ corresponds

to $20 \mathrm{~h}_{50}^{-1} \mathrm{Mpc}$ in terms of Hubble flow. Since it is highly unlikely that the filaments would be so nicely oriented along our line of sight with A3128/A3125, we conclude that their distension in velocity is unlikely to be a reflection of Hubble flow, but rather is due to a tidally-induced velocity gradient in an infalling group. Furthermore, it was demonstrated in Caldwell \& Rose (1997) that tidal distension of subclusters occurs only after the subcluster has passed through the main cluster, in either a head-on, or slightly off-center, encounter. Consequently, we expect that the tidally distended filements have already made a passage through the H-R Supercluster and A3128/A3125 system and are now emerging from that passage. In contrast, the more circularly symmetric (in both position and velocity) groups are probably still in the infall phase of their passage through the cluster/supercluster. Consequently, the filaments are more likely to be associated with visible signs of transient merging activity in the A3128/A3125 system, especially in regard to disturbances in the ICM. To further assess this hypothesis regarding groups versus filaments, it would be of interest to compare the physical properties of the galaxies in groups versus filaments for indications of different recent evolutionary histories.

\subsection{Small-Scale Structure in the Hot ICM}

Over length scales $\lesssim 100 \mathrm{kpc}$, and group membership less than a few galaxies, galaxies are of limited use as tracers of substructure in A3128/A3125. At this point, X-ray emission from the ICM becomes our primary means to delineate small-scale structure. As has been discussed in $\S 3$, ample evidence is seen in the Chandra image of A3128 for structures in the ICM on scales below $1 \mathrm{~h}_{50}^{-1} \mathrm{Mpc}$. We begin by briefly recapitulating those key observations related to the structure of the ICM, namely:

(1) The X-ray emission shows two distinct regions with high surface brightness cores, and an additional lower surface brightness third component between the two main components.

(2) The two bright components have small core radii.

(3) The bright peaks, especially the NE one, are displaced with respect to their underlying lower brightness contours.

(4) In addition to the overall displacement of brighter versus fainter contours, the NE X-ray component shows a strongly asymmetric morphology.

(5) The heavy element abundance of the gas in the NE X-ray component $(\sim 0.13$ solar $)$ is signifi- 
cantly lower than for the gas in the SW component $(\sim 0.6)$.

(6) A bright galaxy (and member of A3128) is coincident with the SW X-ray peak; no bright galaxy coincides with the NE peak.

(7) Radio sources are coincident with both X-ray peaks.

We now attempt to produce a coherent picture of the X-ray structures, and their relation to the optical galaxy and radio data. We will first argue that the X-ray structures are transient, and likely associated with merger activity. Then we propose that the NE X-ray peak is the leading edge of highly supersonic gas, once associated with the F1 and G1 galaxy filament/group, which has now passed through the center of A3128. Finally, we argue that the SW X-ray peak is most likely a gaseous component at the trailing end of the F1/G1 infall pattern which is still at least partially bound to the underlying potential well associated with a compact group of galaxies.

\subsubsection{Transient Nature of the ICM}

The most conspicuous aspect of the ICM is the division of the X-ray emission into two bright peaks, separated by $1.2 \mathrm{~h}_{50}^{-1} \mathrm{Mpc}$ along roughly the same NE-SW axis that joins A3128 with A3125. A third fainter and less centrally concentrated component is located between the NE and SW bright peaks, and very close to the center of the galaxy distribution. The existence of multiple X-ray components on relatively small spatial scales implies transient disturbances in the ICM. In addition, the two bright components have core radii of $\sim 30 \mathrm{~h}_{50}^{-1} \mathrm{kpc}$. While most clusters typically have core radii 10 times greater than this, there are two kinds of clusters with small core radii. First are the cooling flow clusters, which are generally characterized by a cool peak of bright emission atop a relaxed ICM with large core radius (Mohr, Mathiesen, \& Evrard 1999). An example of such a cooling flow cluster with highly structured X-ray emission and a small core radius is A1795 (Fabian et al. 2000). Since the central temperature does not drop in either the NE or SW peak, nor is the underlying cluster relaxed (given the double/triple nature of the X-ray emission and the extensive evidence for ongoing merging in A3128/A3125), the identification of the bright X-ray components in A3128 with cooling flows is clearly inappropriate. The second type of cluster with small core radii is represented by those, such as A548, which are undergoing merging events (Davis et al. 1995). The similarity between A3128 and A548 is striking, in that A548 also has multiple

$\mathrm{X}$-ray emission peaks, with small core radii. In this case the small core radii and low value of $\beta$ is considered a result of the truncation of the cluster profile (Mulchaey 2000; Navarro, Frenk, \& White 1996; Bartelmann \& Steinmetz 1996). Finally, given that the sound travel time across the asymmetric $20 \mathrm{kpc} \mathrm{NE}$ region for a $4 \mathrm{keV}$ gas is only $\sim 2 \times 10^{7}$ years, then unless the gas in the NE emission peak is pressure confined, we are witnessing a highly transient structure in the ICM. We argue in the Appendix A.2 that the group gas has been stripped from the group and thus is no longer gravitationally bound, but is presently ram pressure confined. Furthermore, the NE component is elongated by a $\sim 2: 1$ axis ratio along the same NE-SW axis that separates the two 
bright components. Thus we observe a preferred alignment along the NE-SW from scales below $20 \mathrm{~h}_{50}^{-1} \mathrm{kpc}$ to the $6 \mathrm{Mpc}$ distance separating A3128 from A3125; alignments over a large variety of scales is seen in other clusters as well (West \& Blakeslee 2000). These large-scale alignments are believed to represent the preferred axis of infall and merging activity in the cluster.

\subsubsection{Connection Between X-ray Structure and Merging Galaxy Groups}

Having established the transient nature of the X-ray structures and its probable relation to merging events, we now attempt to link the major X-ray structures with specific spatial/kinematic substructures in the galaxies. First, it is natural to associate the double X-ray structure with the A3128/A3125 double system. However, the projected separation between A3128 and A3125, at $\sim 6 \mathrm{~h}_{50}^{-1} \mathrm{Mpc}$, is approximately 5 times larger than the observed separation between the two high surface brightness X-ray peaks. Furthermore, the small velocity difference between A3125 and A3128 implies a multi-Gyr timescale for that passage, whereas the timescales for the two X-ray peaks (e.g., the cooling timescale, which is only $\sim 1$ Gyr) appear incompatible. Hence we look to smaller substructures in the galaxies for an explanation of the X-ray peaks.

In examining the position-position and position-cz plots in Figs. 7 and 6, the two substructures that are at least projected close to the positions of the bright X-ray peaks are the group denoted by green annuli (G1 in Table 3) and the filament denoted by the red squares (F1 in Table 3). If F1 has been correctly identified as a post-passage (through A3128) tidally distended group, then

it represents the most natural candidate for producing the transient structure seen in the Chandra image of the ICM. Referring to Fig. 7 it is evident that the projected axes of F1 and the NE-SW X-ray sources are approximately parallel. Moreover, since the distinction between F1 and G1 has been drawn somewhat artificially, we henceforth refer to them collectively as the F1/G1 group. A closer look at Fig. 7 reveals that the F1/G1 group extends $\sim 30^{\prime}$ to the NE of the NE X-ray peak, and that this represents the high velocity, hence likely leading, edge of the group. Given our interpretation of the filaments as post-passage tidally distended groups, we argue that the NE end of F1 has passed through the center of A3128, and that much of the group gas is continuing through, with some of it trailing behind, as indicated by the elongated isophotes. On the other hand, the bulk of G1 and the gas associated with the SW X-ray peak are located on the other side of the cluster center, and thus are considered to be still infalling.

\subsubsection{Analysis of the NE X-ray Component}

Support for the above scenario comes from the morphology of the NE peak - in particular, its displacement by more than $2^{\prime}$, or $200 \mathrm{~h}_{50}^{-1} \mathrm{kpc}$, to the NE of the lower surface brightness isophotes of that X-ray component. Given that the large acceleration imparted by the H-R Supercluster should result in supersonic impacts of infalling groups into the main cluster, we can expect to 
observe signs of shock structures in the ICM. The large offset nature of the NE peak may well represent such activity, in which case the outward direction of its motion from the cluster center has been established, in agreement with our hypothesis about the outward flow of the NE end of F1. Further, from the projected separation between the NE end of F1 and the outer edge of the NE X-ray core we can derive an estimate of the relative velocities of the F1 filament and the X-ray source. This calculation is discussed in Appendix A.1. Here we simply report the main result, which is that the gas in the NE X-ray peak is inferred to be travelling at about half the velocity of the leading edge of $\mathrm{F} 1$, and its velocity with respect to the A3128 cluster is $\sim 3500 \mathrm{~km} \mathrm{~s}^{-1}$. This speed corresponds to a Mach Number, $\mathrm{M}=6$, for a typical cluster gas temperature of $3 \mathrm{keV}$. Thus, as discussed in Appendix A.2, the gas in the NE peak is inferred to be highly supersonic, shocked gas which has been stripped from a group of galaxies (now seen as F1) during a passage through A3128 at hypersonic velocity (in excess of $4000 \mathrm{~km} \mathrm{~s}^{-1}$ ). As is discussed in Appendix A.3, at the high Mach Number $(\mathrm{M}=6)$ inferred, the X-ray structure should be primarily determined by the

effects of ram pressure (which is of course axisymmetric) as opposed to cluster thermal pressure (which is spherically symmetric). This is clearly consistent with the observed axial structure in the $\mathrm{X}$-ray source.

One further piece of observational support for this scenario comes from the aforementioned radio arc, which lies slightly to the $\mathrm{NE}$ of the peak emission in the $\mathrm{NE}$ core, i.e., where a bowshock is expected if the gas is moving hypersonically. As mentioned previously, however, deeper radio imaging is required to verify whether or not the radio arc is indeed related to the cluster, rather than to a background source.

\subsubsection{Analysis of the $S W X$-ray Component}

The most intriguing aspect of the X-ray structure is the existence of two bright peaks. The question that naturally arises is whether both peaks can be attributed to a single merging event or whether two separate events must be invoked. The answer to this question is of great significance to interpreting the events taking place in A3128. On the one hand, our analysis has uncovered evidence for a variety of substructures in A3128. On the other hand, only the F1 and G1 groups appear to be in a region coincident with the main X-ray activity, and it is not even clear whether F1 and G1 are two independent systems.

Earlier we interpeted the displacement of the core emission in the NE X-ray component from the lower surface brightness emission in terms of a ram pressure that delineates the direction of motion of the NE peak. In principle, the same argument can be applied to the SW core, to infer that its direction of motion, relative to the main cluster gas, is to the SW. Such an inference would present serious problems for the general scenario presented above, since we have argued that the entire F1/G1 is moving to the NE relative to A3128, with the SW part of it at lower speed. However, we propose instead that the displacement of the SW X-ray core is due to the effect of the local gravitational potential well of a compact group centered on the bright galaxy (ENACS\#75) 
that is coincident with the SW X-ray core and the weak MOST radio source. A CTIO 0.9-m R band image of the region immediately surrounding ENACS\#75 is shown in Fig. 27. There it can be seen that ENACS\#75 itself, which appears as a single bright object (at the contrast shown) on the SuperCOSMOS grayscale image in Fig. 17, coincident with the SW X-ray core, is resolved into three objects, with several more galaxies to the East. The Chandra SW X-ray core component coincides with the main ENACS\#75 object. While we have velocities only for ENACS\#75, and for one other galaxy in the putative compact group, both of these (V=19252 for ENACS\#75 and $\mathrm{V}=18380$ for ENACS\#78) are consistent with the low velocity end of F1 and the mean velocity of G1. The main point is that ENACS\#75 is displaced to the West of the compact group, thus forming a local distortion to the potential well, and perhaps explaining the displacement of the Xray core. Similarly asymmetric X-ray contours, which appear to follow the galaxy mass distribution, have been found in small groups of galaxies (Mulchaey et al. 1996; Davis et al. 1996; Mulchaey 2000). The compact group also provides a plausible explanation for the existence of the SW X-ray component itself. In this scenario the SW component reflects the nature of the ICM of an infalling group that may be associated with the F1/G1 system, but has not yet interacted with the A3128 ICM. Since it has not yet passed though A3128, its ICM is likely still bound in the group, and reflects the local structure in that potential well. By way of contrast, the lack of association between the NE component and any prominent enhancement in the galaxy distribution, as well as its more disturbed morphology, is consistent with its identification as stripped gas from the leading group of galaxies in the F1 filament.

\subsubsection{Comparison with Other Cluster Merging Events}

Further insight regarding the merging event(s) in A3128/A3125 can be gained by comparing the X-ray image of A3128 to high-resolution Chandra imaging of other clusters with merging events. In several clusters, observed with Chandra, signatures of ongoing merging events have been established. In particular, merging events have been studied in the clusters A2142 (Markevitch et al. 2000), A3667 (Vikhlinin, Markevitch, \& Murray 2000a), and RXJ1720.1+2638 (Mazzotta et al. 2001). In

all three cases, the merging event appears to consist of a subcluster "cold" front moving into the ICM of a main cluster. Due to the lack of a pressure and temperature inversion across the front, the inference is that the infalling subcluster is moving subsonically. This represents a marked contrast to the situation in A3128, where we see no evidence that the temperature drop sin the NE and SW components, and where we have inferred that the gravitational influence of the H-R Supercluster has produced hypersonic infall velocities. Thus the A3128/A3125 system potentially represents a different regime of merger activity. A more extensive temperature map of the cluster would be very useful in comparing with the "cold" front clusters. 


\subsubsection{Alternate Scenario}

Thus far we have assumed that all of the diffuse X-ray emission seen in the Chandra ACIS-I image is due to gas associated with the merging events in A3128/A3125. The spatial coincidence between the gas in the SW component and the compact galaxy group in that area, coupled with the coincidence between the peak in the SW emission and the luminous galaxy ENACS\#75 provides circumstantial evidence that the gas in the SW component is, indeed, affiliated with A3128. The redshift of 0.065 found for the SW component gas (see $\S 4$ ) provides conclusive proof of its association with the cluster complex. As well, the redshift of 0.059 found for the diffuse component gas confirms its association with A3128. In the case of the NE component, no such coincidence occurs with a particular galaxy group. The strongest circumstantial evidence for a connection between the gas in the NE component with A3128 is the elongated morphology of the emission, that coincides well with the position angle of the A3128/A3125/F1 merger axis, as well as the asymmetric morphology of the outer isophotes, which taken together suggest a direction of motion of the NE component along the main cluster merger axis. However, barring conclusive redshift information on the X-ray gas, the possibility that the gas could instead be a projection of a background cluster should be considered. In that regard, we again consider the nature of the radio source(s) in the NE component, and of the background galaxy that is coincident with the double-lobed source resolved in the ATCA 13 $\mathrm{cm}$ data. As seen in the CTIO 0.9-m optical R band image, in Fig.23, the background galaxy is the brightest of a number of faint red objects in the field. If we assume that the faint fuzz surrounding that galaxy represents the extended envelope of a $\mathrm{cD}$ galaxy, i.e., that it is the dominant galaxy of a background cluster, then by also assuming a typical absolute magnitude for a $\mathrm{cD}$ of -22 , we infer a redshift of $\mathrm{z} \sim 0.3$. The curved blue feature to the southwest of this putative $\mathrm{cD}$ galaxy might then be seen as a gravitationally lensed arc. Then, if the X-ray emission in the NE is assumed to be associated with this background cluster, its inferred luminosity is $\sim 3 \times 10^{45} \mathrm{erg} / \mathrm{sec}$ in the 0.5 - $10 \mathrm{keV}$ passband. While this is a high luminosity, it is not inconsistent with that of the most X-ray luminous clusters known (e.g., Ebeling et al. 1996; Wu, Xue, \& Fan 1999). In addition, while the observed global temperature of the NE component, which is $\sim 4 \mathrm{keV}$, is low compared to the typical $\sim 8 \mathrm{keV}$ temperature expected for such an X-ray luminous cluster, there is enough scatter in

the $\mathrm{L}_{x}-\mathrm{T}$ relation that a $\sim 4 \mathrm{keV}$ temperature is not out of the question for the $\sim 3 \times 10^{45} \mathrm{erg} / \mathrm{sec}$ luminosity (Wu, Xue, \& Fan 1999).

The key to placing the X-ray gas in the NE component is to obtain a conclusive measure of the redshift using the X-ray emission lines present in the spectrum. As was summarized in §4, our best fit model produces a redshift of 0.10 , with a $90 \%$ confidence of 0.037 to 0.23 . Thus the redshift data is more consistent with $\mathrm{A} 3128$, at $\mathrm{z}=0.06$, than with the proposed background cluster at $\mathrm{z} \sim 0.3$, but is not conclusive. 


\section{Conclusions}

We have collected optical redshift data for 532 objects in the field of the merging double cluster galaxies A3128/A3125. The redshift information has been supplemented by both X-ray and radio imaging. The goal of this program is to characterize and understand the variety of structures still present in the double cluster, and its relation to the larger environment of the HorologiumReticulum Supercluster.

Our principal conclusion is that a large number of substructures are still present in the A3128/A3125 system. This conclusion is based both on groups and filaments evident in the positionvelocity space of the galaxy distribution and on the multi-component nature of the X-ray emitting ICM. The most striking large-scale feature in the galaxy distribution is the relatively low number density of galaxies for $\sim 4000 \mathrm{~km} \mathrm{~s}^{-1}$ on either side of the mean cluster velocity at $\sim 17500 \mathrm{~km} \mathrm{~s}^{-1}$ . We interpret this feature as due to the large gravitational potential well of the H-R Supercluster. Within the $4000 \mathrm{~km} \mathrm{~s}^{-1}$ "depleted" zone, those galaxies that are present appear to be members of small groups or filaments. The latter are extended features in position-velocity space whose statistical reality is somewhat uncertain. We ascribe the filaments to the tidal distension of a group after it has fallen through the A3128/A3125 system and is emerging out the other side. In fact, A3125 itself shows some characteristics of a tidally disturbed system. We note that the filamentary structures appear to follow the main NE-SW axis along which most merging activity appears to be taking place. This is the axis which connects A3125 to the larger A3128 (at a projected separation

of $\sim 6 \mathrm{Mpc}$, and also along which the X-ray emission is split into two bright components, at a projected separation of $\sim 1 \mathrm{Mpc}$.

We have proposed two ongoing merging events to explain the current state of the A3128/A3125 system. The first is the merger event between A3125 and A3128, which is most plausibly explained if A3125 has already passed through A3128, and is highly dispersed as a result of the passage. The second involves the double peaked nature of the X-ray emission. Here we have noticed the close spatial correspondence between one particular high velocity filament and group with the X-ray emitting gas. Although the group/filament system is not massive, in comparison with the main body of A3128, the high infall velocity generated by the potential well of the H-R Supercluster produces a large energy deposition in the collision between the filament and A3128, and allows for only a modest infalling system to produce a major impact on the cluster ICM. Specifically, we argue that the morphology of the NE X-ray peak, along with its coincidence with the higher velocity end of the galaxy filament, indicates that the NE X-ray component represents the surviving ICM of the galaxy filament that has endured a hypersonic ( Mach 6) encounter with A3128. The SW X-ray component appears to be the still intact ICM of a compact group that represents the still-infalling end of the galaxy filament-group. This gas is believed to be responding to the potential well of a compact group of galaxies surrounding a bright galaxy. While the details of this picture are still quite uncertain, the key ingredient is the high encounter velocity produced by the H-R Supercluster, which makes even the infall of a small group an energetic and interesting event in the life of a galaxy cluster. Further observations which could clarify this picture include deeper X-ray observations, to 
characterize the temperature structure in the ICM, deep high resolution radio imaging to clarify the nature of the putative radio arc associated with the NE X-ray peak, redshifts of galaxies in the apparent compact group that is coincident with SW X-ray component, and further redshift surveys throughout the H-R Supercluster.

We wish to thank the staff of the AAT for acquiring the $2 \mathrm{dF}$ data for us under the service observing program and for the significant help given to us in all phases of the observations. The MOST is operated by the University of Sydney and supported in part by grants from the Australian Research Council. The Australia Telescope is funded by the Commonwealth of Australia for operation as a national facility managed by CSIRO. This research has been partially supported by NSF grant AST-9900720 to the University of North Carolina. MJH acknowledges the financial support of a Physics \& Mathematical Physics Scholarship at the University of Adelaide.

\section{A. APPENDIX}

\section{A.1. Infall Velocity of the NE X-ray Component}

As has been discussed in $\S 6$, the F1 galaxy filament (and probably associated G1 group) represents the strongest candidate for producing the transient structure seen in the Chandra image of the ICM. If we assume that F1 is indeed responsible for the NE and SW X-ray components, then a comparison of the projected separation between the NE end of F1 and the outer edge of the NE $\mathrm{X}$-ray core can yield an estimate of the relative velocities of the F1 filament and the X-ray sources. If the X-ray structure was generated at the time when the F1 group first entered the A3128 cluster, then the angular separation is

$$
\Delta \alpha=\left(v_{F 1}-v_{N E}\right) t / D_{A 3128},
$$

where $t$ is the time elapsed since the entry into $\mathrm{A} 3128, v_{F 1}$ is the average velocity of $\mathrm{F} 1, v_{N E}$ is the average velocity of the leading edge of the NE X-ray core, and $D_{A 3128}$ is the distance to the cluster. From Figure 7, the NE "head" of F1 is separated from the leading edge of the NE X-ray source by $\Delta \alpha \sim 30^{\prime}$.

The point at which F1 first entered the A3128 cluster is uncertain because A3128 does not have a sharp boundary, however, it appears that the SW edge of the A3128 galaxy distribution (c.f. Figure 7) is about 15 ' beyond the SW X-ray lobe. Thus, the projected angular distance traveled by the "head" of F1 since entering A3128 is

$$
\alpha_{F 1}=v_{F 1} t / D_{A 3128}=60^{\prime} .
$$

From the ratio of these two angular separations we can therefore estimate that the average velocity of the NE leading edge of the X-ray source is about $1 / 2$ the average velocity of the leading edge of the F1 group. 
The radial velocity of the "head" of $\mathrm{F} 1$ is about $4000 \mathrm{~km} \mathrm{~s}^{-1}$ relative to the A3128 rest frame which implies that the radial velocity of the X-ray structure is about $2000 \mathrm{~km} \mathrm{~s}^{-1}$ (again, relative to the A3128 rest frame). Assuming a random inclination with respect to the plane of the sky then implies that the observed X-ray structure has a velocity relative to the A3128 cluster of $\sim 3500 \mathrm{~km} \mathrm{~s}^{-1}$. This corresponds to a supersonic Mach Number, M=6, for a typical cluster gas temperature of $3 \mathrm{keV}$. This is obviously consistent with the observed axial structure in the X-ray source.

\section{A.2. Stripping of Gas from the NE X-ray Component}

We therefore hypothesize that the NE component of the A3128 X-ray source(s) consists of shocked gas which has been stripped from a group of galaxies (now seen as the F1 filament) that encountered and passed through A3128 at hypersonic velocity (in excess of $4000 \mathrm{~km} \mathrm{~s}^{-1}$ ). In this scenario, as the F1 group approaches the A3128 cluster the gas held in the core of the F1 group is shocked by the encounter with the gas bound to A3128. A bow shock forms and simultaneously a reverse shock propagates back through the F1 gas. This shock system is responsible for decelerating and removing the gas from the F1 group and depositing it in the A3128 cluster.

The velocity of the reverse shock relative to the F1 group is given by the usual shock jump conditions,

$$
v_{R S}^{2}=((\gamma+1) / 2 \gamma)\left(p_{R S} / \rho_{F 1}(\vec{x})\right)
$$

where $\gamma$ is the ideal gas adiabatic index, $v_{R S}$ is the velocity of the reverse shock relative to the F1 group, $p_{R S}$ is the pressure in the reverse shock and $\rho_{F 1}(\vec{x})$ is the local density of the unshocked gas in the F1 group at a particular point. The region between the bow shock and the reverse shock, however, is nearly isobaric so the reverse shock pressure is

$$
p_{R S}=p_{B S}=\left(2 \gamma /(\gamma+1) \rho_{A 3128} v_{F 1}^{2}\right.
$$

where $p_{B S}$ is the bow shock pressure, $\rho_{A 3128}$ is the ambient density in Abell 3128 , and $v_{F 1}$ is the incoming velocity of the F1 group (relative to the barycenter of A3128). As a result there is a simple relation between the reverse shock velocity and the incoming F1 group velocity, i.e.

$$
v_{R S}^{2}=\left(\rho_{A 3128} / \rho_{F 1}(\vec{x})\right) v_{F 1}^{2}
$$

If this reverse shock velocity is greater than escape velocity for the F1 precursor group, then the gas behind the shock front will be stripped from the F1 group and eventually merge with the gas in A3128. In that case, the initial velocity of the stripped gas relative to A3128 is simply the difference between the F1 group velocity and the reverse shock velocity, i.e.

$$
v_{S T R I P}=v_{F 1}-v_{R S}=v_{F 1}\left(1-\left(\rho_{A 3128} / \rho_{F 1}(\vec{x})\right)^{1 / 2}\right)
$$

Note that the velocity of the stripped gas relative to A3128 depends critically on the density ratio, 
$\rho_{A 3128} / \rho_{F 1}(\vec{x})$. If $\rho_{A 3128} / \rho_{F 1}(\vec{x}) \geq 1$ it simply means that the gas in $\mathrm{F} 1$ will be stopped and deposited on the outer perimeter of $\mathrm{A} 3128$ as the $\mathrm{F} 1$ group begins to penetrate $\left(v_{\text {STRIP }}<0\right)$. A negative stripping velocity indicates that the reverse shock gas actually has a net (small) velocity opposite to $v_{F 1}$. This mass of rapidly stripped lower density gas may contribute to the central X-ray component, or it may be trapped in the potential well associated with the G1 group. If, however, there is a high density core in the initial F1 gas distribution, as would be the case for an isothermal density distribution, then the $\rho_{A 3128} / \rho_{F 1}(\vec{x})$ ratio for this high density component would likely be less than 1 . This high density gas would still be strongly decelerated (and thus displaced) from the F1 group, but would continue to penetrate into A3128 at a high (supersonic) velocity as given above for $\rho_{A 3128} / \rho_{F 1}<1$. Clearly, in this case $0<v_{S T R I P}<v_{F 1}$. In other words, as F1 enters and passes through A3128, its gas content is essentially peeled away in layers corresponding to the quiescent density of the gas initially in the core of F1, leaving a trail of stripped gas in its wake. The above estimate that the initial velocity of the shocked, dense core gas represented by the NE X-ray component is about $1 / 2$ of the velocity of $\mathrm{F} 1$ then implies that $\rho_{A 3128} / \rho_{F 1}<1 / 4$ for the central (highest) density in the core.

\section{A.3. Ram Pressure Confinement of the NE Core Gas}

As the reverse shock passes through the core of F1, the core gas decelerates and is displaced from the group of galaxies. In addition, the bow shock wraps around the slower moving shocked gas from the core center and establishes the standard condition for ram pressure confinement, i.e.

$$
\rho_{A 3128} v(t)^{2}=\rho_{C N T} C_{S}^{2}
$$

where $v(t)$ is the bulk velocity of the central core gas relative to A3128, $\rho_{C N T} \simeq 4 \rho_{F 1}(0)$ is the density of the shocked central gas, and $C_{S}$ is the sound speed of this core gas. Note that the initial value for $v(t)$ is equal to $v_{S T R I P}$ for $\rho_{F 1}(\vec{x})=\rho_{F 1}(0)$, i.e., the central density in the unshocked gas.

A quantitative indication of ram pressure confinement is provided by the observed asymmetry in the NE X-ray core, with the highest surface brightness contours displaced toward the outer edge of the emission. This is a standard signature of ram pressure confinement and deceleration. Furthermore, the axially elongated structure of the NE core can be explained in the context of a ram pressure confinement model by the following argument. Ram pressure confinement controls the transverse expansion of the X-ray source, but it does not control the axial expansion as the core gas backfills its trailing wake cavity at the speed of sound. The ratio of these two expansion rates is

$$
\dot{L}_{\text {TRANSVERSE }} / \dot{L}_{P A R A L L E L}=2 \dot{R} / C_{S}=\xi^{-1 / 2}\left(v_{S T R I P} / v(t)\right)^{3 / 4}
$$

where $\dot{R}$ is simply the time derivative of the transverse radius, $v_{S T R I P}$ is the initial velocity of the ram pressure confined central core gas relative to $\mathrm{A} 3128$, and $\xi=\rho_{C N T} / \rho_{A 3128}$ is the density ratio. For the NE component, using the above estimate that $\rho_{A 3128} / \rho_{F 1}(0)<1 / 4$ and $\rho_{C N T} / \rho_{F 1}(0) \sim 4$ 
we conclude that $\xi>16$. Since the X-ray emitting cloud is slowing down, $v_{S T R I P} / v(t)$ is increasing with time, so the observed value of $\dot{L}_{T R A N S V E R S E} / \dot{L}_{P A R A L L E L}=1 / 2$ in conjunction with our estimate that $\xi>16$ implies that the present velocity of the X-ray core has been reduced to about $40 \%$ of its initial value.

\section{A.4. Dynamical versus Radiative Timescales}

Neglecting radiative cooling for the moment, one can estimate the dynamical timescale for the X-ray components using the standard ram pressure confinement arguments (Christiansen 1969),

$$
t_{D Y N}=(8 / 3) \xi\left(R_{I} / v_{S T R I P}\right)
$$

$R_{I}$ is the initial transverse radius of the X-ray core. For the NE component, taking $R_{I} \sim 26 \mathrm{kpc}$ and $v_{S T R I P} \sim 3500 \mathrm{~km} \mathrm{~s}^{-1}$, the dynamical timescale for the NE component is

$$
t_{D Y N}=2.3 \times 10^{7} \xi \mathrm{yr}
$$

For the NE component, using the above estimate that $\rho_{A 3128} / \rho_{F 1}(0)<1 / 4$ and $\rho_{C N T} / \rho_{F 1}(0) \sim 4$ we conclude that $\xi>16$ so

$$
t_{D Y N}>3.2 \times 10^{8} \mathrm{yr}
$$

The cooling time for the hot cluster gas is

$$
\left.t_{C O O L}=\left(8.5 \times 10^{10}\right)\left(\frac{n_{P}}{10^{-3} \mathrm{~cm}^{-3}}\right)^{-1} \frac{T_{g}}{10^{8} \mathrm{~K}}\right)^{1 / 2}(\mathrm{yr})
$$

(Sarazin 1986), where $n_{P}$ is the proton density and $T_{g}$ is the temperature of the gas in Kelvins.

From the central peaks of the two components we estimate that the central densities of the two X-ray components are $n_{N E} \sim 1.1 \times 10^{-2}$ and $n_{S W} \sim 2.4 \times 10^{-2}$. Thus cooling times in these components are $t_{N E}=1.4 \times 10^{9} \mathrm{yr}$ and $t_{S w}=6.5 \times 10^{8} \mathrm{yr}$. Although these cooling times are greater than the dynamical timescale, they are at most a factor of 2-4 larger, hence radiative losses may be an important dynamical factor in slowing the expansion of the X-ray sources as they decelerate (c.f., Christiansen, Rolison, \& Scott 1979).

\section{REFERENCES}

Anders, E. \& Grevesse N. 1989, Geochimica et Cosmochimica Acta, 53, 197

Arnaud, K.A., 1996, in Astronomical Data Analysis Software and Systems V, eds. Jacoby G. and Barnes J., ASP Conf. Series volume 101, 17

Baldi, A., Bardelli, S., \& Zucca, E. 2001, MNRAS, 324, 509

Bardelli, S., Zucca, E., \& Baldi, A. 2001, MNRAS, 320, 387

Bartelmann, M., \& Steinmetz, M. 1996, MNRAS, 283, 431 
Benson, A. J., Pearce, F. R., Frenk, C. S., Baugh, C. M., \& Jenkins, A. 2001, MNRAS, 320, 261

Biviano, A., Katgert, P., Mazure, A., Moles, M., den Hartog, R., Perea, J., \& Focardi, P. 1997, A\&A, 321, 84

Christiansen, W. 1969, MNRAS, 145, 327

Christiansen, W. A., Rolison, G. G., \& Scott, J. S. 1979, ApJ, 234, 456

Davis, D. S., Bird, C. M., Mushotzky, R. F., \& Odewahn, S. C. 1995, ApJ, 440, 48

Davis, D. S., Mulchaey, J. S., Mushotzky, R. F., \& Burstein, D. 1996, ApJ, 460, 601

Dressler, A. 1980, ApJS, 42, 565

Ebeling, H., Voges, W., Bohringer, H., Edge, A. C., Huchra, J. P., \& Briel, U. G. 1996, MNRAS, 281,799

Kauffmann, G., Colberg, J., Diaferio, A., \& White, S. D. M. 1999a, MNRAS, 303, 188

Kauffmann, G., Colberg, J., Diaferio, A., \& White, S. D. M. 1999b, MNRAS, 307, 529

de Lapparent, V., Geller, M. J., \& Huchra, J. P. 1986, ApJ, 302, L1

Einasto, M., Einasto, J., Tago, E., Dalton, G. B., \& Andernach, H. 1994, MNRAS, 269, 301

Ensslin, T. A., Biermann, P. L., Klein, U., \& Kohle, S. 1998, A\&A, 281, 799

Ettori, S., Bardelli, S., De Grandi, S., Molendi, S., Zamorani, G., \& Zucca, E. 2001, MNRAS, in press

Fabian, A. C., Sanders, J. S., Ettori, S., Taylor, G. B., Allen, S. W., Crawford, C. S., Iwasawa, K., \& Johnstine, R. M. 2000, Astro-ph/0011547

Geller, M. J., Diaferio, A., \& Kurtz, M. J. 1999, ApJ, 517, L23

Govoni, F., Feretti, L., Giovannini, G., Bohringer, H., Reiprich, T. H., \& Murgia, M. 2001, A\&A, 376,803

Gregory, S. A., \& Thompson, L. A. 1978, ApJ, 222, 784

Hambly, N. C., Miller, L., MacGillivray, H. T., Herd, J. T., \& Cormack, W. A. 1998, MNRAS, 298, 897

Hambly, N. C., MacGillivray, H. T., Read, M. A., Tritton, S. B., Thomson, E. B., Kelly, B. D., Morgan, D. H., Smith, R. E., Driver, S. P., Williamson, J., Parker, Q. A., Hawkins, M. R. S., Williams, P. M., \& Lawrence, A. 2001, MNRAS, 326, 1279

Hudson, M. J., Smith, R. J., Lucey, J. R., Schlegel, D. J., \& Davies, R. L. 1999, ApJ, 512, L79

Jones, C., \& Forman, W. 1984, ApJ, 276, 38

Jones, L. A. 1999, PhD Thesis, University of North Carolina

Kaiser, N. 1987, MNRAS, 227, 1

Katgert, P., Mazure, A., Perea, J., den Hartog, R., et al. 1996, A\&A, 310, 8 
Katgert, P., Mazure, A., den Hartog, R., Adami, C., Biviano, A., \& Perea, J. 1998, A\&A, 331, 493

Lewis, I., et al. 2000, MNRAS, submitted

Liedahl, D. A., Osterheld, A. L. \& Goldstein, W. H. 1995 ApJ, 438, L115

Lucey, J. R., Dickens, R. J., Mitchell, R. J., \& Dawe, J. A. 1983, MNRAS, 203, 545

Markevitch, M., et al. 2000, ApJ, 541, 542

Mazzotta, P., Markevitch, M., Vikhlinin, A., Forman, W. R., David, L. P., \& VanSpeybroek, L. 2001, ApJ, 555, 205

Mewe, R., Gronenschild, E.H.B.M. \& van den Oord, G.H.J 1985, A\&A, 62, 197

Mewe, R., Lemen, J. R., van den Oord, G.H.J 1986, A\&A, 65, 511

Mohr, J. J., Mathiesen, B. \& Evrard, A. E. 1999, ApJ, 517, 627

Mulchaey, J. S. 2000, ARA\&A, 38, 289

Mulchaey, J. S., Davis,D. S., Mushotzky, R.F., \& Burstein, D. 1996, ApJ, 456, 80

Navarro, J. F., Frenk, C. S., \& White, S. D. M. 1996, MNRAS, 257720

Pearce, F. R., Jenkins, A., Frenk, C. S., White, S. D. M., Thomas, P. A., Couchman, H. M. P., Peacock, J. A., \& Efstathiou, G. 2001, MNRAS, 326, 649

Peres, C. B., Fabian, A. C., Edge, A. C., Allen, S. W., Johnstone, R. M., \& White, D. A. 1998, MNRAS, 298, 416

Raychaudhury, S. 1989, Nature, 342, 251

Robertson, J.G. 1991, Aust. J. Phys. 44, 729

Roettiger, K., Burns, J. O., \& Stone, J. M. 1999, ApJ, 518, 603

Sarazin, C. L., 1986, Reviews of Modern Physics, 58, 1

Sault, R.J., Teuben, P.J., \& Wright, M.C.H. 1995, in ASP Conf. Ser. 77, Astronomical Data Analysis Software and Systems IV, ed. R. Shaw, H.E. Payne, \& J.J.E. Hayes (San Francisco: ASP), 433

Shandarin, S. F. 1983, SvAL, 9, 104

Shapley, H. 1930, Harvard Coll. Obs. Bull., 874, 9

Somerville, R. S., \& Primack, J. 1999, MNRAS, 310, 1087

Venturi, T., Bardelli, S., Zambelli, G., Morganti, R., \& Hunstead, R. W. 2001, MNRAS, in press

Vikhlinin, A., Markevitch, M, \& Murray, S. S. 2000, ApJ, 551, 160

West, M. J., Jones, C., \& Forman, W. 1995, ApJ, 451, L5

West, M. J., \& Blakeslee, J. P. 2000, ApJ, 543, L27

Wu, X.-P., Xue, Y.-J., \& Fang, L.-Z. 1999, ApJ, 524, 22 
Zucca, E., Zamorani, G., Scaramella, R., \& Vettolani, G. 1993, ApJ, 407, 470

This preprint was prepared with the AAS LATEX macros v5.0. 
Fig. 1.- Comparison of the deredshifted $2 \mathrm{dF}$ and Argus spectra of the galaxies \#481 and \#477 in the vicinity of the $[\mathrm{OII}] \lambda 3727$ emission line. Spectra (a) and (b) are the Argus and 2dF spectra for galaxy \#481, while (c) and (d) are $2 \mathrm{dF}$ and Argus spectra for galaxy \#477. The continua of the $2 \mathrm{dF}$ and Argus spectra do not match well due to the fact that the $2 \mathrm{dF}$ spectra are not flux-calibrated.

Fig. 2.- Dec versus RA plot for all galaxies in our A3128/A3125 sample with redshifts in the range $9000<\mathrm{cz}<29000 \mathrm{~km} \mathrm{~s}^{-1}$, with galaxies in the more restricted range $16000<\mathrm{cz}<19500$ $\mathrm{km} \mathrm{s}^{-1}$ marked as filled squares. The positions are given in arcminutes from the center of A3128 ( $\alpha=$ 03:30:43.8 $\delta=-52: 31: 30 \mathrm{~J} 2000$ ). The two bright X-ray peaks are denoted as six-pointed stars.

Fig. 3.- Dec (top) and RA (bottom) are plotted versus redshift for our A3128/A3125 sample. Positions are in arcminutes from the center of A3128.

Fig. 4. - Dec (top) and RA (bottom) are plotted versus redshift for our A3128/A3125 sample. Positions are in arcminutes from the center of A3128. A more restricted redshift range is plotted than in Fig. 3. Various foreground and background redshift slices, discussed later in the text, are identified with different colors and symbol types.

Fig. 5.- Histogram of redshifts in the A3128/A3125 field. The major redshift peak for the A3128/A3125 double cluster is clearly evident at $\sim 17750 \mathrm{~km} \mathrm{~s}^{-1}$, as well as the peaks at \pm 4000 $\mathrm{km} \mathrm{s}^{-1}$ of the mean A3128/A3125 redshift, and the peak at $\sim 33000 \mathrm{~km} \mathrm{~s}^{-1}$. 
Fig. 6.- Position-velocity plots with a number of groups and filaments identified. Red and blue squares are filaments F1 and F2 respectively, while the green and magenta unfilled circles are groups G1 and G2.

Fig. 7.- RA versus Dec position-position plot with the groups and filaments identified in Fig. 6 plotted with the same symbols and colors as in that Figure.

Fig. 8.- Position-velocity plots with a statistical "control" group identified.

Fig. 9.- Position-position plot with the same statistical "control" group identified as in Fig. 8.

Fig. 10.- Position-velocity plots with a number of small groups identified. The cyan, magenta, and blue open circles are groups G3, G6, and G7, while the green and red squares are G4 and G5.

Fig. 11.- RA versus Dec position-position plot with the small groups identified in Fig. 10 plotted with the same symbols and colors as in that Figure.

Fig. 12.- RA versus Dec position-position plot with the different redshift slices identified in Fig. 4 plotted with the same symbols and colors as in that Figure.

Fig. 13. - Galaxy redshifts are plotted versus their radial distance from the center of A3128. Again, the $\sim 4000 \mathrm{~km} \mathrm{~s}^{-1}$ "depletion" zone on either side of the mean cluster redshift can be seen. 
Fig. 14. - Chandra ACIS-I image of A3128, in the energy range $0.5-10 \mathrm{keV}$, displayed at a contrast level to emphasize the cores of the two high surface brightness components. The horizontal axis gives the full $16^{\prime}$ field of the ACIS-I image. The image has been binned by $4 \times 4$ pixels, and then smoothed with a gaussian of $\sigma=4.0$ pixels.

Fig. 15.- Chandra ACIS-I image of A3128, in the energy range $0.5-10 \mathrm{keV}$, displayed at a contrast level to emphasize the lower surface brightness features in the image, notably the third X-ray component that lies between the two bright emission peaks. The horizontal axis gives the full 16' field of the ACIS-I image. The image has been binned by $4 \times 4$ pixels, and then smoothed with a gaussian of $\sigma=4.0$ pixels. The gaps between the four individual CCD I0 - I3 chips, can be seen in the plot, despite the dithering of the observation.

Fig. 16. - Adaptively smoothed Chandra image of A3128, showing the $0.5-10 \mathrm{keV}$ emission contours overlaid on a grayscale representation of the image. The displacement of the NE and SW X-ray peaks from their fainter contours can be clearly seen. The full $16^{\prime} \times 16^{\prime}$ of the ACIS-I image is shown.

Fig. 17.- Contours from the Chandra ACIS-I image overlaid on a grayscale representation of the SuperCOSMOS digitized image of the $\mathrm{B}_{J}$ bandpass UK Schmidt plate. The Chandra contour levels are $0.3,0.4,0.5,0.7$, and 1.0 counts/sec in the smoothed image.

Fig. 18. - Radial surface brightness profiles are plotted for both the NE (top) and SW (bottom) X-ray peaks versus Radius (left panels) and $\log (\mathrm{R})$ (right panels).

Fig. 19.- X (left panels) and Y (right panels) centers of the ellipses fitted to the X-ray surface brightness in the NE (top) and SW (bottom) components. The X-Y coordinates, which are RA and Dec respectively, are given in pixels, where each pixel corresponds to 2".

Fig. 20.- Ellipticity (left panels) and position angle (right panels) profiles are plotted versus semi-major axis for the NE (top) and SW (bottom) components. 
Fig. 21. - Contours of the MOST $843 \mathrm{MHz}$ radio emission are overlaid on a grayscale representation of the Chandra ACIS-I image of A3128. The MOST contour levels are 2, 3, 5, 7, 10, 15, 20, 30, 50, 70 , and $100 \mathrm{mJy} /$ beam, where the beamsize is $54^{\prime \prime} \mathrm{x} 43^{\prime \prime}$ at position angle $0^{\circ}$.

Fig. 22. - Contours of the MOST $843 \mathrm{MHz}$ radio emission are overlaid on a grayscale representation of the optical SuperCOSMOS digital $B_{J}$ image. MOST contour levels are the same as in Fig. 21.

Fig. 23. - CTIO 0.9-m telescope images in the B (left) and R (right) bandpasses of the faint galaxy associated with the radio source in the NE X-ray component. Exposure times for both images were 15 minutes. The field of view is $2^{\prime} \times 2^{\prime}$. The curved feature to the southwest of the galaxy is most evident in the B image. North is to the top and East to the left.

Fig. 24. - Radio contours from the ATCA data are overplotted on the CTIO $0.9 \mathrm{~m}$ optical R band image. The $20 \mathrm{~cm}$ (blue) and $13 \mathrm{~cm}$ (red) contours are coincident with a faint galaxy. The higher resolution $13 \mathrm{~cm}$ data clearly show the double lobed structure of the radio source. Both radio images use uniform weighting. The $20 \mathrm{~cm}$ beam is $10.2^{\prime \prime} \times 6.3^{\prime \prime}\left(\mathrm{PA}-11^{\circ}\right)$ and the $13 \mathrm{~cm}$ beam is $5.2^{\prime \prime} \times 3.2^{\prime \prime}$ $\left(\mathrm{PA}-11^{\circ}\right)$. The $20 \mathrm{~cm}$ contour levels are $0.5,1,2,4$, and $8 \mathrm{mJy} /$ beam. The $13 \mathrm{~cm}$ contour levels are $2,4,6$, and $8 \mathrm{mJy} /$ beam.

Fig. 25.- MOST (green) and ATCA $20 \mathrm{~cm}$ (blue) radio contours and Chandra X-ray (red) contours of the NE X-ray peak are overlaid on the CTIO 0.9-m R band image of A3128. The beam size and orientation of the $20 \mathrm{~cm}$ image, which is $15.0^{\prime \prime} \times 9.95^{\prime \prime}\left(\mathrm{PA}-20^{\circ}\right)$, is shown as the black ellipse in the lower right corner of the figure. The ATCA contour levels are 1, 2, 4, 8, and 16 mJy/beam. The MOST contours are 2, 3, 4, 7, 10, 20, and $40 \mathrm{mJy} /$ beam, while the beamsize is $54^{\prime \prime} \times 43^{\prime \prime}\left(\mathrm{PA} 0^{\circ}\right)$. The Chandra contours are 0.14, 0.16, 0.22, 0.32, 0.45, 0.63, 0.85, 1.10, 1.39, 1.73 , and 2.10 counts/sec in the smoothed image.

Fig. 26. - Histogram of all redshifts in the A3128/A3125 field from $5000 \mathrm{~km} \mathrm{~s}^{-1}$ out to 65000 $\mathrm{km} \mathrm{s}^{-1}$. The distribution in redshift is evidently nonuniform out to the limit of the sample.

Fig. 27.- CTIO 0.9-m $\mathrm{R}$ band image of the $\sim 2.5^{\prime}$ region surrounding the bright galaxy ENACS\#75, showing the apparent compact group of galaxies that lies mostly to the east of it. ENACS\#75 resolves into the triple object in the center of the image. 
Table 1. Summary of the Radio Observations

\begin{tabular}{ccccccc}
\hline \hline Date & $\begin{array}{c}\text { Frequency } \\
(\mathrm{MHz})\end{array}$ & Config. & $\begin{array}{c}\text { Baselines } \\
(\mathrm{m})\end{array}$ & $\begin{array}{c}\text { Pointing centre } \\
(\mathrm{J} 2000)\end{array}$ & $\begin{array}{c}t_{\mathrm{obs}} \\
(\mathrm{hr})\end{array}$ \\
\hline 1991 Dec 03 & 843 & MOST & $15-1600$ & $033012.4-523348$ & 12.0 \\
1999 Feb 28 & $1384 / 2496$ & $6 \mathrm{C}$ & $153-6000$ & $033115.0-524148$ & 1.67 \\
2000 Mar 23 & $1384 / 2496$ & $6 \mathrm{D}$ & $77-5878$ & $033115.0-524148$ & 1.15 \\
2000 Nov 04 & $1384 / 2496$ & $6 \mathrm{D}$ & $77-5878$ & $033115.0-524148$ & 0.65 \\
\hline
\end{tabular}


Table 2. Velocity Data from 2dF Galaxy Spectra

\begin{tabular}{|c|c|c|c|c|c|c|c|c|c|c|c|c|}
\hline \multirow{2}{*}{$\frac{\text { ID }}{1}$} & \multirow{2}{*}{ Other ID } & \multicolumn{3}{|c|}{$\alpha(\mathrm{J} 2000)$} & \multicolumn{3}{|c|}{$\delta(\mathrm{J} 2000)$} & \multirow{2}{*}{$\frac{\mathrm{B}_{J}}{16.8}$} & \multirow{2}{*}{$\frac{\mathrm{cZ}}{18365}$} & \multirow{2}{*}{$\frac{\text { ref }}{a}$} & \multirow{2}{*}{$\frac{\sigma_{V}}{103.5}$} & \multirow{2}{*}{$\frac{\text { Emission }}{\mathrm{n}}$} \\
\hline & & 03 & 22 & 53.67 & -52 & 39 & 51.1 & & & & & \\
\hline 3 & & 03 & 23 & 01.99 & -52 & 58 & 42.2 & 18.3 & 26219 & e & 98.1 & $\mathrm{y}$ \\
\hline 5 & & 03 & 23 & 10.56 & -53 & 05 & 08.1 & 17.7 & 17682 & $\mathrm{a}$ & 32.4 & $\mathrm{n}$ \\
\hline 6 & & 03 & 23 & 15.56 & -52 & 53 & 44.1 & 17.3 & 17573 & $\mathrm{a}$ & 37.1 & $\mathrm{n}$ \\
\hline 7 & & 03 & 23 & 20.91 & -52 & 26 & 20.5 & 18.4 & 34195 & e & 68.9 & $\mathrm{y}$ \\
\hline 8 & & 03 & 23 & 23.45 & -53 & 08 & 09.2 & 17.8 & 9877 & e & 189.7 & $\mathrm{y}$ \\
\hline 9 & & 03 & 23 & 26.54 & -52 & 29 & 03.5 & 16.9 & 21977 & $\mathrm{ae}$ & 63.9 & $\mathrm{y}$ \\
\hline 10 & & 03 & 23 & 38.52 & -53 & 13 & 48.7 & 16.4 & 17951 & $\mathrm{a}$ & 42.4 & $\mathrm{y}$ \\
\hline 11 & & 03 & 23 & 38.72 & -53 & 18 & 32.7 & 18.4 & 32721 & $\mathrm{ae}$ & 45.9 & $\mathrm{y}$ \\
\hline 13 & & 03 & 23 & 41.41 & -53 & 10 & 00.5 & 18.1 & 322 & $\mathrm{a}$ & 48.5 & $\mathrm{n}$ \\
\hline 15 & & 03 & 23 & 42.75 & -52 & 42 & 52.6 & 17.0 & 23583 & $\mathrm{a}$ & 263.6 & $\mathrm{n}$ \\
\hline 16 & & 03 & 23 & 43.41 & -53 & 19 & 16.9 & 17.8 & 17859 & e & 161.1 & $\mathrm{y}$ \\
\hline 17 & & 03 & 23 & 44.20 & -53 & 15 & 25.1 & 17.7 & 19591 & $\mathrm{a}$ & 71.2 & $\mathrm{y}$ \\
\hline 19 & & 03 & 23 & 44.57 & -53 & 17 & 32.7 & 16.6 & 17134 & $\mathrm{ae}$ & 239.9 & $\mathrm{y}$ \\
\hline 21 & & 03 & 23 & 44.59 & -53 & 20 & 46.8 & 16.7 & 23350 & ae & 39.9 & $\mathrm{y}$ \\
\hline 25 & & 03 & 23 & 45.33 & -52 & 50 & 27.4 & 17.2 & 23026 & $\mathrm{ae}$ & 58.1 & $\mathrm{y}$ \\
\hline 29 & & 03 & 23 & 51.31 & -53 & 19 & 04.9 & 17.2 & 17790 & $\mathrm{a}$ & 132.6 & $\mathrm{y}$ \\
\hline 32 & & 03 & 23 & 59.84 & -52 & 43 & 26.7 & 16.4 & 21935 & $\mathrm{ae}$ & 63.9 & $\mathrm{y}$ \\
\hline 33 & & 03 & 24 & 00.13 & -52 & 34 & 23.6 & 16.9 & 119 & $\mathrm{a}$ & 35.6 & $\mathrm{n}$ \\
\hline 35 & & 03 & 24 & 01.67 & -53 & 08 & 18.5 & 16.9 & 17604 & $\mathrm{ae}$ & 98.5 & $\mathrm{y}$ \\
\hline 36 & & 03 & 24 & 04.33 & -52 & 22 & 51.4 & 17.9 & 19437 & e & 89.8 & $\mathrm{y}$ \\
\hline 38 & & 03 & 24 & 04.67 & -53 & 18 & 53.9 & 17.7 & 23122 & $\mathrm{ae}$ & 104.6 & $\mathrm{y}$ \\
\hline 40 & & 03 & 24 & 07.26 & -52 & 49 & 43.2 & 17.4 & 24009 & $\mathrm{ae}$ & 104.0 & $\mathrm{y}$ \\
\hline 42 & & 03 & 24 & 12.05 & -53 & 20 & 32.9 & 15.9 & 17808 & ae & 46.7 & $\mathrm{y}$ \\
\hline 47 & & 03 & 24 & 20.70 & -52 & 57 & 35.5 & 18.4 & 17719 & $\mathrm{a}$ & 95.5 & $\mathrm{n}$ \\
\hline 48 & & 03 & 24 & 24.35 & -52 & 42 & 48.4 & 16.3 & 18211 & ae & 82.3 & $\mathrm{y}$ \\
\hline 50 & & 03 & 24 & 26.47 & -52 & 38 & 04.6 & 17.4 & 21971 & $\mathrm{ae}$ & 97.6 & $\mathrm{y}$ \\
\hline 53 & & 03 & 24 & 30.52 & -52 & 49 & 35.0 & 18.2 & -130 & $\mathrm{a}$ & 229.8 & $\mathrm{n}$ \\
\hline 54 & & 03 & 24 & 33.76 & -53 & 07 & 42.5 & 18.3 & 32638 & $\mathrm{ae}$ & 130.0 & $\mathrm{y}$ \\
\hline 56 & & 03 & 24 & 35.16 & -52 & 37 & 24.5 & 17.6 & 23206 & $\mathrm{ae}$ & 102.2 & $\mathrm{y}$ \\
\hline 58 & & 03 & 24 & 38.33 & -52 & 49 & 48.9 & 18.2 & 18306 & $\mathrm{a}$ & 62.1 & $\mathrm{n}$ \\
\hline 59 & & 03 & 24 & 42.40 & -52 & 33 & 53.8 & 17.3 & 14007 & e & 84.5 & $\mathrm{y}$ \\
\hline 60 & & 03 & 24 & 45.31 & -53 & 25 & 45.2 & 16.2 & 17716 & $\mathrm{ae}$ & 44.0 & $\mathrm{y}$ \\
\hline
\end{tabular}


Table 2-Continued

\begin{tabular}{|c|c|c|c|c|c|c|c|c|c|c|c|c|}
\hline \multirow{2}{*}{$\frac{\mathrm{ID}}{62}$} & \multirow{2}{*}{ Other ID } & \multicolumn{3}{|c|}{$\alpha(\mathrm{J} 2000)$} & \multicolumn{3}{|c|}{$\delta(\mathrm{J} 2000)$} & \multirow{2}{*}{$\frac{\mathrm{B}_{J}}{15.6}$} & \multirow{2}{*}{$\frac{\mathrm{cz}}{18}$} & \multirow{2}{*}{$\frac{\text { ref }}{a}$} & \multirow{2}{*}{$\frac{\sigma_{V}}{13.0}$} & \multirow{2}{*}{$\frac{\text { Emission }}{\mathrm{n}}$} \\
\hline & & 03 & 24 & 46.45 & -53 & 02 & 09.9 & & & & & \\
\hline 64 & & 03 & 24 & 47.90 & -53 & 22 & 33.0 & 17.9 & 17894 & $\mathrm{a}$ & 42.2 & $\mathrm{y}$ \\
\hline 66 & & 03 & 24 & 49.04 & -52 & 39 & 10.8 & 18.2 & 52673 & e & 109.9 & y \\
\hline 68 & & 03 & 24 & 57.48 & -53 & 23 & 10.8 & 17.9 & 16701 & $\mathrm{ae}$ & 68.5 & $\mathrm{y}$ \\
\hline 69 & & 03 & 24 & 58.47 & -53 & 24 & 58.9 & 16.3 & 17956 & $\mathrm{ae}$ & 44.8 & $\mathrm{y}$ \\
\hline 70 & & 03 & 25 & 02.21 & -52 & 08 & 40.1 & 16.8 & 21324 & $\mathrm{a}$ & 75.4 & $\mathrm{n}$ \\
\hline 71 & & 03 & 25 & 02.94 & -53 & 14 & 38.3 & 17.5 & 24084 & $\mathrm{a}$ & 62.6 & y \\
\hline 73 & & 03 & 25 & 03.22 & -52 & 43 & 33.2 & 18.2 & 22072 & $\mathrm{a}$ & 58.7 & $\mathrm{y}$ \\
\hline 74 & & 03 & 25 & 03.81 & -52 & 48 & 44.6 & 17.9 & 18351 & $\mathrm{a}$ & 126.7 & $\mathrm{n}$ \\
\hline 75 & & 03 & 25 & 07.82 & -53 & 19 & 10.8 & 18.3 & 17966 & $\mathrm{ae}$ & 122.6 & $\mathrm{y}$ \\
\hline 76 & & 03 & 25 & 11.28 & -52 & 33 & 12.6 & 17.7 & 18227 & $\mathrm{ae}$ & 48.0 & $\mathrm{y}$ \\
\hline 77 & & 03 & 25 & 12.75 & -52 & 17 & 11.5 & 17.3 & 23119 & $\mathrm{ae}$ & 57.5 & $\mathrm{y}$ \\
\hline 78 & & 03 & 25 & 16.36 & -53 & 35 & 38.5 & 16.9 & 17825 & $\mathrm{ae}$ & 69.1 & $\mathrm{y}$ \\
\hline 79 & & 03 & 25 & 17.04 & -52 & 51 & 44.8 & 16.3 & 13813 & $\mathrm{ae}$ & 68.4 & y \\
\hline 80 & & 03 & 25 & 18.49 & -53 & 21 & 28.7 & 17.0 & 17996 & ae & 182.1 & $\mathrm{y}$ \\
\hline 81 & & 03 & 25 & 18.99 & -52 & 43 & 42.4 & 17.5 & 21835 & $\mathrm{a}$ & 76.9 & $\mathrm{y}$ \\
\hline 82 & & 03 & 25 & 20.10 & -52 & 32 & 05.8 & 16.9 & 18181 & $\mathrm{ae}$ & 47.1 & $\mathrm{y}$ \\
\hline 83 & & 03 & 25 & 20.86 & -52 & 06 & 29.3 & 17.9 & 18175 & $\mathrm{a}$ & 46.7 & $\mathrm{n}$ \\
\hline 84 & & 03 & 25 & 22.32 & -53 & 24 & 40.9 & 17.7 & 17651 & $\mathrm{ae}$ & 62.8 & $\mathrm{y}$ \\
\hline 85 & & 03 & 25 & 22.45 & -52 & 12 & 09.2 & 17.2 & 20750 & ae & 89.7 & $\mathrm{y}$ \\
\hline 87 & & 03 & 25 & 27.03 & -52 & 57 & 54.4 & 17.4 & 31826 & e & 108.3 & $\mathrm{y}$ \\
\hline 88 & & 03 & 25 & 33.01 & -52 & 39 & 27.3 & 18.5 & 39719 & ae & 219.1 & $\mathrm{y}$ \\
\hline 89 & & 03 & 25 & 35.66 & -52 & 34 & 54.1 & 18.2 & 18343 & ae & 225.0 & $\mathrm{y}$ \\
\hline 94 & & 03 & 25 & 41.72 & -52 & 55 & 04.3 & 18.5 & 176 & $\mathrm{a}$ & 109.9 & $\mathrm{n}$ \\
\hline 95 & & 03 & 25 & 42.50 & -52 & 31 & 00.0 & 16.0 & 18901 & $\mathrm{a}$ & 37.7 & $\mathrm{y}$ \\
\hline 96 & & 03 & 25 & 44.42 & -52 & 32 & 58.2 & 16.1 & 18360 & $\mathrm{ae}$ & 52.1 & $\mathrm{y}$ \\
\hline 97 & & 03 & 25 & 45.24 & -52 & 51 & 45.4 & 18.3 & 17347 & e & 58.6 & $\mathrm{y}$ \\
\hline 99 & & 03 & 25 & 46.62 & -53 & 28 & 53.7 & 17.4 & 32695 & ae & 148.6 & $\mathrm{y}$ \\
\hline 100 & & 03 & 25 & 47.75 & -52 & 53 & 32.8 & 17.5 & 18078 & e & 76.1 & $\mathrm{y}$ \\
\hline 101 & & 03 & 25 & 49.68 & -53 & 23 & 03.6 & 17.0 & 19117 & ae & 111.3 & $\mathrm{y}$ \\
\hline 102 & & 03 & 25 & 51.06 & -52 & 16 & 22.4 & 17.5 & 21152 & $\mathrm{a}$ & 68.0 & $\mathrm{n}$ \\
\hline 104 & & 03 & 25 & 58.52 & -53 & 10 & 19.4 & 18.0 & 22853 & e & 197.2 & $\mathrm{y}$ \\
\hline 105 & & 03 & 26 & 00.95 & -52 & 10 & 37.7 & 17.8 & 16436 & e & 93.5 & $\mathrm{y}$ \\
\hline
\end{tabular}


Table 2-Continued

\begin{tabular}{|c|c|c|c|c|c|c|c|c|c|c|c|c|}
\hline \multirow{2}{*}{$\frac{\mathrm{ID}}{106}$} & \multirow{2}{*}{ Other ID } & \multicolumn{3}{|c|}{$\alpha(\mathrm{J} 2000)$} & \multicolumn{3}{|c|}{$\delta(\mathrm{J} 2000)$} & \multirow{2}{*}{$\frac{\mathrm{B}_{J}}{16.2}$} & \multirow{2}{*}{$\frac{\mathrm{cZ}}{18354}$} & \multirow{2}{*}{$\frac{\text { ref }}{\mathrm{ae}}$} & \multirow{2}{*}{$\frac{\sigma_{V}}{23.0}$} & \multirow{2}{*}{$\begin{array}{c}\text { Emission } \\
\mathrm{y}\end{array}$} \\
\hline & & 03 & 26 & 01.07 & -53 & 26 & 06.3 & & & & & \\
\hline 109 & & 03 & 26 & 02.42 & -52 & 08 & 04.5 & 18.2 & 39381 & $\mathrm{a}$ & 177.4 & $\mathrm{n}$ \\
\hline 110 & & 03 & 26 & 03.59 & -52 & 35 & 55.5 & 17.7 & 18017 & e & 217.4 & y \\
\hline 113 & & 03 & 26 & 09.69 & -53 & 10 & 51.9 & 17.7 & 23206 & $\mathrm{ae}$ & 52.9 & $\mathrm{y}$ \\
\hline 114 & & 03 & 26 & 10.22 & -52 & 54 & 49.1 & 18.1 & 31781 & $\mathrm{a}$ & 16.7 & $\mathrm{n}$ \\
\hline 115 & & 03 & 26 & 10.24 & -52 & 34 & 56.9 & 16.8 & 25113 & $\mathrm{a}$ & 206.1 & $\mathrm{y}$ \\
\hline 117 & & 03 & 26 & 13.02 & -52 & 28 & 37.9 & 16.9 & 18194 & $\mathrm{a}$ & 43.0 & $\mathrm{n}$ \\
\hline 118 & & 03 & 26 & 15.69 & -53 & 20 & 45.1 & 17.0 & 19370 & $\mathrm{a}$ & 52.5 & $\mathrm{n}$ \\
\hline 119 & & 03 & 26 & 18.02 & -52 & 49 & 09.1 & 17.1 & 17390 & e & 86.4 & $\mathrm{y}$ \\
\hline 120 & & 03 & 26 & 18.50 & -53 & 32 & 47.3 & 15.8 & 18870 & $\mathrm{ae}$ & 70.3 & $\mathrm{y}$ \\
\hline 121 & & 03 & 26 & 21.60 & -52 & 55 & 01.3 & 18.2 & 31792 & e & 56.5 & $\mathrm{y}$ \\
\hline 122 & & 03 & 26 & 23.13 & -52 & 39 & 43.3 & 16.5 & 18049 & $\mathrm{ae}$ & 74.5 & $\mathrm{y}$ \\
\hline 123 & & 03 & 26 & 26.16 & -52 & 12 & 29.4 & 17.7 & 17807 & e & 164.7 & $\mathrm{y}$ \\
\hline 124 & & 03 & 26 & 26.41 & -52 & 30 & 24.8 & 17.7 & 17759 & $\mathrm{a}$ & 86.3 & $\mathrm{n}$ \\
\hline 126 & & 03 & 26 & 27.05 & -53 & 28 & 58.6 & 17.8 & 18330 & e & 59.2 & $\mathrm{y}$ \\
\hline 127 & & 03 & 26 & 30.91 & -53 & 37 & 34.9 & 17.3 & 18792 & $\mathrm{a}$ & 245.2 & $\mathrm{y}$ \\
\hline 128 & & 03 & 26 & 31.94 & -53 & 39 & 04.6 & 16.5 & 18563 & $\mathrm{ae}$ & 69.8 & $\mathrm{y}$ \\
\hline 129 & & 03 & 26 & 32.10 & -52 & 59 & 31.7 & 16.9 & 31842 & $\mathrm{a}$ & 76.3 & $\mathrm{y}$ \\
\hline 130 & & 03 & 26 & 35.35 & -53 & 20 & 07.0 & 16.9 & 19552 & $\mathrm{ae}$ & 111.5 & $\mathrm{y}$ \\
\hline 131 & & 03 & 26 & 38.53 & -52 & 52 & 46.2 & 16.8 & 18259 & $\mathrm{ae}$ & 119.4 & $\mathrm{y}$ \\
\hline 133 & & 03 & 26 & 40.18 & -52 & 39 & 35.7 & 18.5 & 19110 & e & 243.2 & y \\
\hline 134 & & 03 & 26 & 44.39 & -52 & 00 & 50.6 & 17.9 & 26506 & e & 224.9 & $\mathrm{y}$ \\
\hline 135 & & 03 & 26 & 45.09 & -53 & 05 & 40.8 & 18.3 & 23327 & $\mathrm{e}$ & 103.7 & $\mathrm{y}$ \\
\hline 136 & & 03 & 26 & 47.16 & -52 & 57 & 45.6 & 17.3 & -41 & $\mathrm{a}$ & 90.8 & $\mathrm{n}$ \\
\hline 137 & & 03 & 26 & 50.23 & -53 & 21 & 39.1 & 17.5 & 32354 & $\mathrm{ae}$ & 96.3 & $\mathrm{y}$ \\
\hline 138 & & 03 & 26 & 50.68 & -53 & 31 & 05.2 & 17.1 & 18729 & $\mathrm{a}$ & 46.6 & $\mathrm{n}$ \\
\hline 141 & & 03 & 26 & 56.83 & -52 & 53 & 19.4 & 17.3 & 19606 & $\mathrm{a}$ & 46.7 & $\mathrm{y}$ \\
\hline 142 & & 03 & 26 & 57.99 & -52 & 55 & 51.5 & 18.0 & 22709 & ae & 48.1 & $\mathrm{y}$ \\
\hline 143 & & 03 & 27 & 01.66 & -53 & 09 & 15.6 & 16.9 & 19987 & $\mathrm{a}$ & 58.8 & $\mathrm{y}$ \\
\hline 145 & & 03 & 27 & 03.80 & -53 & 28 & 23.2 & 18.0 & 18825 & e & 52.0 & $\mathrm{y}$ \\
\hline 146 & & 03 & 27 & 05.04 & -53 & 21 & 41.7 & 16.9 & 18693 & e & 116.2 & $\mathrm{y}$ \\
\hline 149 & & 03 & 27 & 08.30 & -52 & 10 & 15.3 & 17.6 & 13441 & $\mathrm{ae}$ & 87.2 & $\mathrm{y}$ \\
\hline 150 & & 03 & 27 & 08.44 & -53 & 37 & 26.3 & 17.5 & 17565 & $\mathrm{ae}$ & 263.8 & $\mathrm{y}$ \\
\hline
\end{tabular}


Table 2-Continued

\begin{tabular}{|c|c|c|c|c|c|c|c|c|c|c|c|c|}
\hline \multirow{2}{*}{$\frac{\mathrm{ID}}{151}$} & \multirow{2}{*}{ Other ID } & \multicolumn{3}{|c|}{$\alpha(\mathrm{J} 2000)$} & \multicolumn{3}{|c|}{$\delta(\mathrm{J} 2000)$} & \multirow{2}{*}{$\frac{\mathrm{B}_{J}}{15.6}$} & \multirow{2}{*}{$\frac{\mathrm{cZ}}{\mathrm{9083}}$} & \multirow{2}{*}{$\frac{\text { ref }}{\mathrm{ae}}$} & \multirow{2}{*}{$\frac{\sigma_{V}}{187.9}$} & \multirow{2}{*}{$\begin{array}{c}\text { Emission } \\
\mathrm{y}\end{array}$} \\
\hline & & 03 & 27 & 10.31 & -53 & 00 & 36.4 & & & & & \\
\hline 152 & & 03 & 27 & 10.53 & -51 & 55 & 51.5 & 18.3 & 60556 & e & 143.5 & $\mathrm{y}$ \\
\hline 153 & & 03 & 27 & 10.85 & -53 & 39 & 46.9 & 14.1 & 17799 & ae & 72.7 & $\mathrm{y}$ \\
\hline 155 & & 03 & 27 & 12.96 & -53 & 02 & 35.1 & 17.5 & 17955 & $\mathrm{ae}$ & 92.9 & $\mathrm{y}$ \\
\hline 156 & & 03 & 27 & 16.88 & -52 & 35 & 03.5 & 18.2 & 18267 & $\mathrm{a}$ & 52.7 & $\mathrm{n}$ \\
\hline 157 & & 03 & 27 & 19.06 & -52 & 47 & 35.3 & 18.1 & 24478 & $\mathrm{a}$ & 397.8 & $\mathrm{n}$ \\
\hline 158 & & 03 & 27 & 20.20 & -53 & 28 & 34.5 & 17.3 & 18476 & $\mathrm{ae}$ & 145.0 & y \\
\hline 159 & & 03 & 27 & 20.35 & -52 & 34 & 14.2 & 18.4 & 19512 & e & 109.8 & $\mathrm{y}$ \\
\hline 165 & & 03 & 27 & 27.70 & -52 & 29 & 14.9 & 17.9 & 19723 & e & 0.0 & $\mathrm{y}$ \\
\hline 168 & & 03 & 27 & 29.36 & -52 & 52 & 48.7 & 17.6 & 22293 & $\mathrm{ae}$ & 135.0 & $\mathrm{y}$ \\
\hline 170 & & 03 & 27 & 30.99 & -52 & 38 & 18.4 & 18.1 & 18489 & $\mathrm{a}$ & 162.8 & $\mathrm{n}$ \\
\hline 172 & & 03 & 27 & 31.09 & -53 & 23 & 40.4 & 17.6 & 19359 & $\mathrm{a}$ & 49.8 & $\mathrm{n}$ \\
\hline 174 & & 03 & 27 & 32.98 & -53 & 20 & 22.3 & 16.5 & 17866 & $\mathrm{a}$ & 69.8 & $\mathrm{y}$ \\
\hline 175 & & 03 & 27 & 33.98 & -53 & 40 & 07.3 & 17.6 & 19005 & $\mathrm{a}$ & 32.8 & $\mathrm{n}$ \\
\hline 178 & & 03 & 27 & 40.10 & -51 & 59 & 22.4 & 18.0 & 17 & $\mathrm{a}$ & 36.8 & $\mathrm{n}$ \\
\hline 180 & & 03 & 27 & 40.38 & -53 & 33 & 31.6 & 17.7 & 17834 & e & 93.7 & $\mathrm{y}$ \\
\hline 181 & & 03 & 27 & 41.83 & -52 & 32 & 49.5 & 17.6 & 19035 & e & 232.1 & $\mathrm{y}$ \\
\hline 186 & & 03 & 27 & 42.76 & -53 & 38 & 23.8 & 17.4 & 17801 & $\mathrm{ae}$ & 118.7 & $\mathrm{y}$ \\
\hline 188 & & 03 & 27 & 42.90 & -53 & 34 & 23.6 & 16.3 & 18430 & $\mathrm{a}$ & 63.3 & $\mathrm{n}$ \\
\hline 191 & & 03 & 27 & 45.63 & -52 & 10 & 41.8 & 18.0 & -20 & $\mathrm{a}$ & 60.5 & $\mathrm{n}$ \\
\hline 193 & & 03 & 27 & 47.26 & -52 & 30 & 04.1 & 17.8 & 9 & $\mathrm{a}$ & 13.1 & $\mathrm{n}$ \\
\hline 195 & & 03 & 27 & 47.91 & -52 & 31 & 21.2 & 18.0 & 18525 & $\mathrm{a}$ & 54.1 & $\mathrm{n}$ \\
\hline 197 & & 03 & 27 & 49.07 & -52 & 24 & 05.9 & 17.0 & 17924 & ae & 40.7 & $\mathrm{y}$ \\
\hline 203 & & 03 & 27 & 52.55 & -53 & 24 & 08.5 & 16.2 & 18405 & $\mathrm{ae}$ & 40.9 & $\mathrm{y}$ \\
\hline 204 & & 03 & 27 & 53.12 & -51 & 53 & 48.2 & 17.3 & -32 & $\mathrm{a}$ & 60.1 & $\mathrm{n}$ \\
\hline 206 & & 03 & 27 & 53.93 & -52 & 23 & 03.8 & 17.8 & 17935 & $\mathrm{a}$ & 34.6 & $\mathrm{n}$ \\
\hline 207 & & 03 & 27 & 53.98 & -53 & 35 & 10.3 & 17.8 & 17974 & $\mathrm{ae}$ & 559.8 & $\mathrm{y}$ \\
\hline 209 & & 03 & 27 & 54.18 & -53 & 46 & 57.3 & 16.7 & 16034 & $\mathrm{a}$ & 27.2 & $\mathrm{n}$ \\
\hline 217 & & 03 & 28 & 03.56 & -52 & 06 & 53.7 & 18.1 & 17264 & e & 16.9 & $\mathrm{y}$ \\
\hline 218 & & 03 & 28 & 03.94 & -53 & 33 & 02.2 & 18.0 & 28250 & ae & 109.9 & $\mathrm{y}$ \\
\hline 220 & & 03 & 28 & 04.41 & -52 & 44 & 42.2 & 18.4 & 18106 & e & 201.6 & $\mathrm{y}$ \\
\hline 223 & & 03 & 28 & 07.49 & -52 & 36 & 50.9 & 18.4 & 16869 & $\mathrm{ae}$ & 62.9 & $\mathrm{y}$ \\
\hline 224 & & 03 & 28 & 08.56 & -52 & 30 & 31.7 & 18.1 & 11808 & $\mathrm{ae}$ & 250.6 & $\mathrm{y}$ \\
\hline
\end{tabular}


Table 2-Continued

\begin{tabular}{|c|c|c|c|c|c|c|c|c|c|c|c|c|}
\hline \multirow{2}{*}{$\frac{\mathrm{ID}}{225}$} & \multirow{2}{*}{$\begin{array}{r}\text { Other ID } \\
\text { E24 }\end{array}$} & \multicolumn{3}{|c|}{$\alpha(\mathrm{J} 2000)$} & \multicolumn{3}{|c|}{$\delta(\mathrm{J} 2000)$} & \multirow{2}{*}{$\frac{\mathrm{B}_{J}}{18.3}$} & \multirow{2}{*}{$\frac{\mathrm{cz}}{17939}$} & \multirow{2}{*}{$\frac{\text { ref }}{\mathrm{e}}$} & \multirow{2}{*}{$\frac{\sigma_{V}}{126.8}$} & \multirow{2}{*}{$\frac{\text { Emission }}{\mathrm{y}}$} \\
\hline & & 03 & 28 & 10.28 & -52 & 48 & 59.7 & & & & & \\
\hline 226 & & 03 & 28 & 11.45 & -53 & 38 & 18.7 & 17.7 & 33310 & $\mathrm{a}$ & 131.1 & $\mathrm{n}$ \\
\hline 228 & & 03 & 28 & 12.82 & -52 & 15 & 59.3 & 18.3 & 10 & $\mathrm{a}$ & 137.5 & $\mathrm{n}$ \\
\hline 229 & & 03 & 28 & 14.03 & -53 & 38 & 34.5 & 17.4 & 33218 & $\mathrm{ae}$ & 201.6 & $\mathrm{y}$ \\
\hline 231 & & 03 & 28 & 15.31 & -52 & 42 & 01.1 & 18.3 & 18031 & $\mathrm{ae}$ & 53.7 & $\mathrm{y}$ \\
\hline 233 & & 03 & 28 & 16.51 & -52 & 13 & 57.6 & 18.4 & 47184 & $\mathrm{a}$ & 29.0 & $\mathrm{n}$ \\
\hline 235 & & 03 & 28 & 17.33 & -51 & 50 & 53.3 & 17.7 & 18572 & e & 137.2 & $\mathrm{y}$ \\
\hline 236 & & 03 & 28 & 17.86 & -53 & 34 & 59.3 & 18.3 & 17084 & $\mathrm{a}$ & 65.0 & $\mathrm{n}$ \\
\hline 237 & & 03 & 28 & 21.35 & -52 & 35 & 19.6 & 16.6 & -43 & $\mathrm{a}$ & 60.7 & $\mathrm{n}$ \\
\hline 240 & & 03 & 28 & 23.20 & -53 & 42 & 32.6 & 18.1 & 21534 & ae & 145.3 & $\mathrm{y}$ \\
\hline 241 & & 03 & 28 & 23.38 & -53 & 34 & 36.0 & 16.2 & 18880 & $\mathrm{ae}$ & 188.7 & $\mathrm{y}$ \\
\hline 242 & & 03 & 28 & 24.53 & -51 & 58 & 52.1 & 17.4 & 18834 & e & 73.1 & $\mathrm{y}$ \\
\hline 245 & & 03 & 28 & 27.46 & -51 & 55 & 30.8 & 17.1 & 17183 & $\mathrm{e}$ & 67.2 & $\mathrm{y}$ \\
\hline 247 & & 03 & 28 & 28.17 & -53 & 27 & 28.0 & 17.6 & 79 & $\mathrm{a}$ & 11.6 & $\mathrm{n}$ \\
\hline 248 & & 03 & 28 & 28.18 & -53 & 47 & 12.7 & 17.4 & 17896 & ae & 61.1 & $\mathrm{y}$ \\
\hline 251 & & 03 & 28 & 30.56 & -53 & 33 & 48.5 & 17.9 & 17730 & $\mathrm{a}$ & 176.6 & $\mathrm{y}$ \\
\hline 253 & E31,CR27a & 03 & 28 & 32.40 & -52 & 57 & 09.7 & 17.2 & 18047 & $\mathrm{a}$ & 174.2 & $\mathrm{n}$ \\
\hline 254 & E30 & 03 & 28 & 32.60 & -52 & 15 & 56.3 & 18.1 & 23130 & e & 109.1 & $\mathrm{y}$ \\
\hline 255 & & 03 & 28 & 33.17 & -53 & 11 & 40.5 & 17.6 & 22844 & e & 91.2 & $\mathrm{y}$ \\
\hline 256 & & 03 & 28 & 34.44 & -53 & 24 & 35.1 & 17.0 & 18125 & ae & 64.1 & $\mathrm{y}$ \\
\hline 257 & & 03 & 28 & 35.08 & -53 & 48 & 43.1 & 18.1 & 33559 & ae & 149.6 & $\mathrm{y}$ \\
\hline 261 & & 03 & 28 & 39.75 & -53 & 19 & 29.2 & 18.3 & 22874 & e & 241.4 & $\mathrm{y}$ \\
\hline 263 & & 03 & 28 & 42.33 & -53 & 29 & 13.6 & 17.7 & 18092 & $\mathrm{a}$ & 95.4 & $\mathrm{n}$ \\
\hline 264 & E33 & 03 & 28 & 44.99 & -53 & 03 & 27.4 & 17.4 & 23038 & ae & 56.6 & $\mathrm{y}$ \\
\hline 267 & E34,CR20a & 03 & 28 & 45.99 & -52 & 58 & 50.5 & 16.8 & 18284 & $\mathrm{a}$ & 265.1 & $\mathrm{n}$ \\
\hline 268 & & 03 & 28 & 46.69 & -52 & 38 & 23.5 & 18.1 & 17084 & $\mathrm{a}$ & 76.2 & $\mathrm{n}$ \\
\hline 270 & & 03 & 28 & 47.45 & -52 & 33 & 59.6 & 18.2 & 18002 & e & 129.5 & $\mathrm{y}$ \\
\hline 271 & E37 & 03 & 28 & 48.73 & -52 & 27 & 18.8 & 17.6 & 17692 & ae & 68.3 & $\mathrm{y}$ \\
\hline 272 & & 03 & 28 & 49.09 & -53 & 27 & 52.0 & 18.1 & 17564 & e & 206.0 & $\mathrm{y}$ \\
\hline 274 & E38 & 03 & 28 & 51.90 & -52 & 13 & 52.1 & 16.7 & 16664 & ae & 41.9 & $\mathrm{y}$ \\
\hline 276 & & 03 & 28 & 53.64 & -52 & 48 & 12.5 & 18.3 & 18235 & $\mathrm{a}$ & 56.9 & $\mathrm{n}$ \\
\hline 277 & E40 & 03 & 28 & 55.01 & -52 & 12 & 58.6 & 17.3 & 18116 & $\mathrm{a}$ & 145.6 & $\mathrm{n}$ \\
\hline 281 & & 03 & 28 & 59.76 & -53 & 22 & 20.4 & 17.8 & 23078 & e & 406.4 & $\mathrm{y}$ \\
\hline
\end{tabular}


Table 2-Continued

\begin{tabular}{|c|c|c|c|c|c|c|c|c|c|c|c|c|}
\hline \multirow{2}{*}{$\frac{\mathrm{ID}}{285}$} & \multirow{2}{*}{$\begin{array}{r}\text { Other ID } \\
\text { E46 }\end{array}$} & \multicolumn{3}{|c|}{$\alpha(\mathrm{J} 2000)$} & \multicolumn{3}{|c|}{$\delta(\mathrm{J} 2000)$} & \multirow{2}{*}{$\frac{\mathrm{B}_{J}}{17.9}$} & \multirow{2}{*}{$\frac{\mathrm{cz}}{16772}$} & \multirow{2}{*}{$\frac{\text { ref }}{a}$} & \multirow{2}{*}{$\frac{\sigma_{V}}{39.8}$} & \multirow{2}{*}{$\frac{\text { Emission }}{\mathrm{n}}$} \\
\hline & & 03 & 29 & 05.94 & -52 & 46 & 32.1 & & & & & \\
\hline 287 & & 03 & 29 & 08.09 & -53 & 42 & 15.4 & 18.1 & 17872 & $\mathrm{e}$ & 108.6 & $\mathrm{y}$ \\
\hline 288 & & 03 & 29 & 09.45 & -52 & 48 & 52.9 & 18.3 & 17427 & $\mathrm{e}$ & 436.8 & $\mathrm{y}$ \\
\hline 290 & E51 & 03 & 29 & 16.51 & -52 & 37 & 38.1 & 15.8 & 18378 & $\mathrm{a}$ & 104.6 & $\mathrm{n}$ \\
\hline 292 & & 03 & 29 & 18.95 & -52 & 12 & 38.7 & 18.5 & 18054 & $\mathrm{a}$ & 68.3 & $\mathrm{n}$ \\
\hline 293 & & 03 & 29 & 20.75 & -52 & 47 & 58.5 & 17.8 & 24720 & $\mathrm{e}$ & 114.3 & $\mathrm{y}$ \\
\hline 294 & E54 & 03 & 29 & 22.12 & -52 & 37 & 10.3 & 15.0 & 11664 & $\mathrm{a}$ & 42.1 & $\mathrm{n}$ \\
\hline 295 & & 03 & 29 & 23.59 & -51 & 56 & 36.4 & 18.3 & 50551 & $\mathrm{a}$ & 228.4 & $\mathrm{n}$ \\
\hline 296 & & 03 & 29 & 24.32 & -53 & 16 & 08.5 & 18.5 & 12005 & ae & 160.6 & $\mathrm{y}$ \\
\hline 297 & & 03 & 29 & 24.73 & -52 & 33 & 02.4 & 18.1 & 89 & $\mathrm{a}$ & 34.2 & $\mathrm{n}$ \\
\hline 300 & E58,CR118a & 03 & 29 & 26.47 & -52 & 30 & 27.6 & 17.0 & 19555 & $\mathrm{a}$ & 39.4 & $\mathrm{n}$ \\
\hline 301 & & 03 & 29 & 27.11 & -52 & 40 & 54.5 & 17.3 & 31884 & ae & 49.5 & $\mathrm{y}$ \\
\hline 305 & & 03 & 29 & 28.56 & -52 & 42 & 36.4 & 17.8 & 16378 & ae & 92.7 & $\mathrm{y}$ \\
\hline 306 & E61,CR167a & 03 & 29 & 29.96 & -52 & 17 & 58.2 & 17.0 & 18076 & $\mathrm{a}$ & 45.1 & $\mathrm{n}$ \\
\hline 310 & E66 & 03 & 29 & 33.38 & -52 & 46 & 33.9 & 17.5 & 16747 & $\mathrm{a}$ & 62.4 & $\mathrm{n}$ \\
\hline 311 & & 03 & 29 & 33.82 & -53 & 45 & 50.5 & 16.8 & 24763 & $\mathrm{a}$ & 55.5 & $\mathrm{y}$ \\
\hline 312 & E68 & 03 & 29 & 34.47 & -52 & 51 & 00.2 & 17.5 & 17104 & $\mathrm{a}$ & 65.9 & $\mathrm{n}$ \\
\hline 313 & & 03 & 29 & 34.92 & -53 & 33 & 03.9 & 17.9 & 17486 & $\mathrm{ae}$ & 38.5 & $\mathrm{y}$ \\
\hline 315 & E67 & 03 & 29 & 35.39 & -52 & 12 & 52.0 & 17.9 & 17848 & $\mathrm{a}$ & 27.2 & $\mathrm{y}$ \\
\hline 316 & & 03 & 29 & 36.01 & -52 & 34 & 16.5 & 18.5 & 19163 & $\mathrm{a}$ & 59.6 & $\mathrm{n}$ \\
\hline 317 & & 03 & 29 & 36.66 & -52 & 29 & 35.0 & 18.1 & 16657 & $\mathrm{a}$ & 194.9 & $\mathrm{n}$ \\
\hline 319 & & 03 & 29 & 37.01 & -53 & 34 & 55.1 & 18.1 & 34900 & $\mathrm{e}$ & 108.9 & $\mathrm{y}$ \\
\hline 320 & E70 & 03 & 29 & 37.80 & -52 & 41 & 55.5 & 16.7 & 17152 & ae & 89.7 & $\mathrm{y}$ \\
\hline 321 & & 03 & 29 & 37.97 & -52 & 49 & 12.8 & 18.3 & 18472 & $\mathrm{a}$ & 303.2 & $\mathrm{n}$ \\
\hline 326 & & 03 & 29 & 41.46 & -52 & 29 & 35.4 & 17.6 & 17511 & ae & 48.3 & $\mathrm{y}$ \\
\hline 327 & E73 & 03 & 29 & 41.57 & -52 & 31 & 16.6 & 18.1 & 18825 & $\mathrm{a}$ & 72.1 & $\mathrm{n}$ \\
\hline 328 & & 03 & 29 & 43.49 & -53 & 15 & 34.3 & 18.5 & 22916 & $\mathrm{e}$ & 380.5 & $\mathrm{y}$ \\
\hline 330 & & 03 & 29 & 45.76 & -52 & 00 & 22.0 & 18.3 & 21270 & $\mathrm{a}$ & 55.5 & $\mathrm{n}$ \\
\hline 331 & & 03 & 29 & 46.62 & -52 & 43 & 51.9 & 18.1 & -16 & $\mathrm{a}$ & 60.9 & $\mathrm{n}$ \\
\hline 332 & & 03 & 29 & 46.70 & -52 & 37 & 05.7 & 18.4 & 18712 & $\mathrm{a}$ & 96.1 & $\mathrm{n}$ \\
\hline 335 & & 03 & 29 & 50.98 & -53 & 27 & 58.9 & 17.2 & 23004 & ae & 46.4 & $\mathrm{y}$ \\
\hline 337 & & 03 & 29 & 51.92 & -52 & 40 & 43.4 & 17.6 & 18383 & $\mathrm{a}$ & 68.7 & $\mathrm{y}$ \\
\hline 341 & E77 & 03 & 29 & 52.85 & -52 & 51 & 55.1 & 17.3 & 17131 & $\mathrm{a}$ & 45.1 & $\mathrm{n}$ \\
\hline
\end{tabular}


Table 2-Continued

\begin{tabular}{|c|c|c|c|c|c|c|c|c|c|c|c|c|}
\hline \multirow{2}{*}{$\frac{\text { ID }}{343}$} & \multirow[t]{2}{*}{ Other ID } & \multicolumn{3}{|c|}{$\alpha(\mathrm{J} 2000)$} & \multicolumn{3}{|c|}{$\delta(\mathrm{J} 2000)$} & \multirow{2}{*}{$\frac{\mathrm{B}_{J}}{17.7}$} & \multirow{2}{*}{$\frac{c z}{27973}$} & \multirow{2}{*}{$\frac{\text { ref }}{a}$} & \multirow{2}{*}{$\frac{\sigma_{V}}{99.6}$} & \multirow{2}{*}{$\frac{\text { Emission }}{\mathrm{n}}$} \\
\hline & & 03 & 29 & 53.54 & -53 & 35 & 33.4 & & & & & \\
\hline 348 & & 03 & 29 & 56.24 & -52 & 37 & 28.2 & 18.3 & 19454 & $\mathrm{a}$ & 331.5 & $\mathrm{n}$ \\
\hline 349 & E80 & 03 & 29 & 56.83 & -53 & 09 & 03.3 & 16.7 & 19920 & e & 201.8 & $\mathrm{y}$ \\
\hline 351 & & 03 & 29 & 59.78 & -53 & 26 & 47.0 & 17.5 & 17904 & $\mathrm{ae}$ & 62.8 & y \\
\hline 352 & & 03 & 29 & 59.88 & -52 & 38 & 13.1 & 18.3 & 19309 & $\mathrm{a}$ & 98.4 & $\mathrm{n}$ \\
\hline 353 & E81 & 03 & 30 & 00.33 & -52 & 18 & 31.8 & 18.1 & 45606 & $\mathrm{a}$ & 140.2 & $\mathrm{n}$ \\
\hline 355 & & 03 & 30 & 03.00 & -52 & 55 & 30.6 & 18.3 & 16761 & $\mathrm{a}$ & 98.2 & $\mathrm{n}$ \\
\hline 361 & E84 & 03 & 30 & 05.04 & -52 & 15 & 25.1 & 17.9 & -29 & $\mathrm{a}$ & 64.8 & $\mathrm{n}$ \\
\hline 365 & & 03 & 30 & 06.11 & -53 & 42 & 37.5 & 15.4 & 15940 & $\mathrm{ae}$ & 43.9 & $\mathrm{y}$ \\
\hline 367 & & 03 & 30 & 08.47 & -53 & 23 & 51.8 & 17.3 & 17826 & e & 122.6 & $\mathrm{y}$ \\
\hline 368 & & 03 & 30 & 09.11 & -53 & 18 & 47.0 & 18.2 & 22630 & e & 100.3 & y \\
\hline 370 & & 03 & 30 & 10.39 & -53 & 45 & 18.6 & 17.4 & 17684 & ae & 105.5 & $\mathrm{y}$ \\
\hline 371 & & 03 & 30 & 10.52 & -53 & 34 & 06.6 & 17.3 & 18419 & e & 65.3 & $\mathrm{y}$ \\
\hline 372 & & 03 & 30 & 10.58 & -52 & 27 & 07.6 & 17.7 & 17386 & $\mathrm{a}$ & 38.5 & $\mathrm{n}$ \\
\hline 373 & E89 & 03 & 30 & 11.13 & -52 & 14 & 51.6 & 17.7 & 44935 & $\mathrm{e}$ & 235.6 & $\mathrm{y}$ \\
\hline 374 & E90 & 03 & 30 & 11.60 & -53 & 09 & 03.5 & 18.0 & 19884 & $\mathrm{ae}$ & 63.4 & $\mathrm{y}$ \\
\hline 376 & & 03 & 30 & 12.81 & -52 & 45 & 04.9 & 18.2 & 17817 & $\mathrm{a}$ & 56.8 & $\mathrm{n}$ \\
\hline 377 & & 03 & 30 & 12.95 & -51 & 52 & 31.3 & 17.4 & 25686 & $\mathrm{a}$ & 236.6 & $\mathrm{n}$ \\
\hline 378 & & 03 & 30 & 13.18 & -53 & 47 & 03.2 & 16.5 & 15833 & e & 179.1 & $\mathrm{y}$ \\
\hline 380 & E94 & 03 & 30 & 13.60 & -53 & 14 & 33.1 & 17.5 & 17506 & $\mathrm{a}$ & 46.0 & $\mathrm{n}$ \\
\hline 388 & & 03 & 30 & 19.46 & -53 & 37 & 03.4 & 17.8 & 17726 & $\mathrm{a}$ & 159.0 & $\mathrm{n}$ \\
\hline 390 & E99,CR130a & 03 & 30 & 22.10 & -52 & 26 & 07.6 & 17.6 & 17739 & $\mathrm{a}$ & 48.5 & $\mathrm{n}$ \\
\hline 393 & E103 & 03 & 30 & 23.11 & -53 & 02 & 58.9 & 18.0 & 22274 & $\mathrm{ae}$ & 115.8 & $\mathrm{y}$ \\
\hline 396 & & 03 & 30 & 24.09 & -52 & 44 & 37.2 & 18.2 & 19884 & $\mathrm{ae}$ & 63.8 & $\mathrm{y}$ \\
\hline 397 & & 03 & 30 & 25.00 & -53 & 36 & 50.7 & 17.7 & 17791 & $\mathrm{a}$ & 111.0 & $\mathrm{n}$ \\
\hline 402 & & 03 & 30 & 32.24 & -52 & 38 & 49.7 & 17.6 & 19651 & $\mathrm{a}$ & 80.9 & $\mathrm{n}$ \\
\hline 403 & & 03 & 30 & 33.78 & -53 & 18 & 40.9 & 17.5 & 14208 & $\mathrm{a}$ & 32.4 & $\mathrm{y}$ \\
\hline 404 & & 03 & 30 & 33.85 & -52 & 23 & 52.6 & 18.4 & 18229 & e & 16.0 & $\mathrm{y}$ \\
\hline 414 & CR128a & 03 & 30 & 39.71 & -52 & 26 & 28.4 & 18.3 & 17405 & $\mathrm{a}$ & 127.6 & $\mathrm{n}$ \\
\hline 415 & & 03 & 30 & 40.99 & -53 & 15 & 34.0 & 17.7 & 17652 & $\mathrm{ae}$ & 189.6 & $\mathrm{y}$ \\
\hline 416 & & 03 & 30 & 41.11 & -52 & 24 & 46.3 & 17.9 & 18689 & $\mathrm{a}$ & 36.6 & $\mathrm{n}$ \\
\hline 422 & & 03 & 30 & 46.35 & -53 & 40 & 16.8 & 17.1 & 18152 & $\mathrm{a}$ & 127.2 & $\mathrm{n}$ \\
\hline 430 & E120 & 03 & 30 & 50.26 & -52 & 14 & 29.9 & 18.2 & 20887 & $\mathrm{a}$ & 48.4 & $\mathrm{n}$ \\
\hline
\end{tabular}


Table 2-Continued

\begin{tabular}{|c|c|c|c|c|c|c|c|c|c|c|c|c|}
\hline \multirow{2}{*}{$\frac{\text { ID }}{431}$} & \multirow[t]{2}{*}{ Other ID } & \multicolumn{3}{|c|}{$\alpha(\mathrm{J} 2000)$} & \multicolumn{3}{|c|}{$\delta(\mathrm{J} 2000)$} & \multirow{2}{*}{$\frac{\mathrm{B}_{J}}{18.4}$} & \multirow{2}{*}{$\frac{\mathrm{cz}}{18542}$} & \multirow{2}{*}{$\frac{\text { ref }}{\text { ae }}$} & \multirow{2}{*}{$\frac{\sigma_{V}}{72.7}$} & \multirow{2}{*}{$\frac{\text { Emission }}{\mathrm{y}}$} \\
\hline & & 03 & 30 & 50.47 & -52 & 18 & 56.5 & & & & & \\
\hline 432 & E124 & 03 & 30 & 50.67 & -52 & 58 & 31.0 & 17.9 & 17968 & $\mathrm{a}$ & 64.4 & $\mathrm{n}$ \\
\hline 434 & & 03 & 30 & 51.28 & -52 & 31 & 15.1 & 17.1 & -62 & $\mathrm{a}$ & 83.6 & $\mathrm{n}$ \\
\hline 438 & & 03 & 30 & 54.01 & -53 & 40 & 36.7 & 17.8 & 15921 & $\mathrm{a}$ & 336.7 & $\mathrm{n}$ \\
\hline 440 & & 03 & 30 & 54.88 & -53 & 37 & 31.0 & 17.9 & 44269 & e & 76.0 & $\mathrm{y}$ \\
\hline 442 & & 03 & 30 & 55.49 & -53 & 35 & 09.7 & 18.4 & 27840 & $\mathrm{a}$ & 114.3 & $\mathrm{n}$ \\
\hline 443 & & 03 & 30 & 55.62 & -51 & 51 & 50.4 & 18.5 & 18134 & e & 265.6 & $\mathrm{y}$ \\
\hline 445 & E131 & 03 & 30 & 56.30 & -53 & 07 & 34.5 & 16.8 & 18538 & ae & 374.9 & $\mathrm{y}$ \\
\hline 447 & & 03 & 30 & 56.49 & -53 & 45 & 49.8 & 16.8 & 15704 & $\mathrm{a}$ & 159.0 & $\mathrm{n}$ \\
\hline 448 & E130 & 03 & 30 & 56.52 & -52 & 51 & 20.0 & 16.8 & 18710 & $\mathrm{ae}$ & 48.8 & $\mathrm{y}$ \\
\hline 449 & E128 & 03 & 30 & 57.02 & -52 & 24 & 32.7 & 18.4 & 18221 & $\mathrm{a}$ & 99.9 & $\mathrm{n}$ \\
\hline 451 & & 03 & 30 & 59.28 & -53 & 41 & 52.8 & 17.0 & 18147 & $\mathrm{ae}$ & 43.0 & $\mathrm{y}$ \\
\hline 456 & & 03 & 31 & 01.65 & -52 & 40 & 50.4 & 18.5 & 19036 & e & 130.2 & $\mathrm{y}$ \\
\hline 458 & E138 & 03 & 31 & 02.14 & -53 & 06 & 06.8 & 18.0 & 18378 & ae & 111.4 & $\mathrm{y}$ \\
\hline 464 & & 03 & 31 & 06.67 & -52 & 30 & 39.0 & 17.8 & 17503 & $\mathrm{a}$ & 43.1 & $\mathrm{n}$ \\
\hline 467 & E143,CR144a & 03 & 31 & 08.18 & -52 & 25 & 03.2 & 17.4 & 18502 & $\mathrm{a}$ & 41.7 & $\mathrm{n}$ \\
\hline 470 & & 03 & 31 & 08.89 & -53 & 19 & 12.3 & 18.0 & 22882 & e & 168.1 & $\mathrm{y}$ \\
\hline 472 & & 03 & 31 & 10.47 & -52 & 55 & 41.2 & 16.9 & 21488 & $\mathrm{ae}$ & 81.5 & $\mathrm{y}$ \\
\hline 473 & E146 & 03 & 31 & 11.06 & -52 & 10 & 33.8 & 17.8 & 18385 & $\mathrm{a}$ & 41.3 & $\mathrm{n}$ \\
\hline 475 & E147 & 03 & 31 & 11.81 & -52 & 26 & 13.8 & 17.8 & 65 & $\mathrm{a}$ & 41.9 & $\mathrm{n}$ \\
\hline 477 & E148,CR80a & 03 & 31 & 12.26 & -52 & 35 & 56.8 & 15.6 & 16946 & ae & 399.1 & $\mathrm{y}$ \\
\hline 478 & & 03 & 31 & 12.99 & -52 & 13 & 14.0 & 18.4 & 21332 & $\mathrm{a}$ & 64.9 & $\mathrm{n}$ \\
\hline 479 & & 03 & 31 & 13.72 & -52 & 04 & 40.2 & 16.4 & 9421 & e & 113.6 & $\mathrm{y}$ \\
\hline 481 & E151,CR53a & 03 & 31 & 14.96 & -52 & 41 & 48.1 & 16.0 & 19936 & $\mathrm{ae}$ & 63.7 & $\mathrm{y}$ \\
\hline 482 & E150,CR156a & 03 & 31 & 15.36 & -52 & 22 & 27.4 & 16.4 & 18070 & $\mathrm{a}$ & 209.0 & $\mathrm{n}$ \\
\hline 483 & CR105a & 03 & 31 & 15.86 & -52 & 30 & 09.9 & 18.1 & 17656 & $\mathrm{a}$ & 24.2 & $\mathrm{n}$ \\
\hline 484 & E152 & 03 & 31 & 18.27 & -52 & 38 & 17.5 & 18.0 & 24327 & e & 72.9 & $\mathrm{y}$ \\
\hline 485 & & 03 & 31 & 18.77 & -52 & 12 & 13.1 & 18.4 & 49771 & ae & 192.4 & $\mathrm{y}$ \\
\hline 486 & & 03 & 31 & 19.97 & -53 & 30 & 30.6 & 18.3 & 18038 & $\mathrm{a}$ & 90.9 & $\mathrm{n}$ \\
\hline 487 & E153 & 03 & 31 & 21.45 & -53 & 01 & 43.6 & 18.0 & 23185 & $\mathrm{a}$ & 68.0 & $\mathrm{n}$ \\
\hline 489 & & 03 & 31 & 23.49 & -53 & 27 & 50.6 & 18.5 & 23247 & $\mathrm{a}$ & 65.9 & $\mathrm{n}$ \\
\hline 490 & & 03 & 31 & 23.73 & -53 & 05 & 13.5 & 18.4 & 19651 & e & 268.5 & $\mathrm{y}$ \\
\hline 491 & & 03 & 31 & 23.85 & -53 & 14 & 36.4 & 17.4 & 22978 & $\mathrm{ae}$ & 210.6 & $\mathrm{y}$ \\
\hline
\end{tabular}


Table 2-Continued

\begin{tabular}{|c|c|c|c|c|c|c|c|c|c|c|c|c|}
\hline \multirow{2}{*}{$\frac{\text { ID }}{492}$} & \multirow{2}{*}{$\begin{array}{r}\text { Other ID } \\
\text { E157 }\end{array}$} & \multicolumn{3}{|c|}{$\alpha(\mathrm{J} 2000)$} & \multicolumn{3}{|c|}{$\delta(\mathrm{J} 2000)$} & \multirow{2}{*}{$\frac{\mathrm{B}_{J}}{18.1}$} & \multirow{2}{*}{$\frac{\mathrm{cz}}{18963}$} & \multirow{2}{*}{$\frac{\text { ref }}{a}$} & \multirow{2}{*}{$\frac{\sigma_{V}}{122.5}$} & \multirow{2}{*}{$\frac{\text { Emission }}{n}$} \\
\hline & & 03 & 31 & 24.80 & -52 & 50 & 30.7 & & & & & \\
\hline 493 & E156,CR126a & 03 & 31 & 25.44 & -52 & 28 & 10.0 & 15.9 & 16755 & $\mathrm{a}$ & 213.0 & $\mathrm{n}$ \\
\hline 494 & & 03 & 31 & 25.44 & -52 & 36 & 16.1 & 17.9 & 16757 & $\mathrm{a}$ & 31.5 & $\mathrm{n}$ \\
\hline 497 & & 03 & 31 & 27.26 & -53 & 01 & 45.7 & 17.7 & 22563 & e & 85.2 & $\mathrm{y}$ \\
\hline 498 & & 03 & 31 & 27.33 & -52 & 30 & 05.3 & 18.0 & 17490 & $\mathrm{a}$ & 43.8 & $\mathrm{n}$ \\
\hline 501 & & 03 & 31 & 30.75 & -52 & 03 & 42.6 & 18.2 & 54493 & e & 122.9 & $\mathrm{y}$ \\
\hline 502 & E159 & 03 & 31 & 31.16 & -52 & 20 & 44.1 & 16.3 & 18499 & $\mathrm{ae}$ & 41.5 & $\mathrm{y}$ \\
\hline 509 & E166 & 03 & 31 & 37.77 & -53 & 00 & 17.8 & 17.0 & 23042 & ae & 74.1 & $\mathrm{y}$ \\
\hline 510 & & 03 & 31 & 38.99 & -52 & 06 & 47.3 & 17.6 & 24991 & $\mathrm{e}$ & 61.7 & $\mathrm{y}$ \\
\hline 512 & & 03 & 31 & 40.42 & -52 & 22 & 23.5 & 18.3 & 17718 & $\mathrm{a}$ & 45.9 & $\mathrm{n}$ \\
\hline 513 & & 03 & 31 & 41.23 & -53 & 07 & 58.8 & 18.3 & 18403 & $\mathrm{ae}$ & 93.2 & $\mathrm{y}$ \\
\hline 514 & E168 & 03 & 31 & 42.30 & -52 & 25 & 11.9 & 16.9 & 19946 & $\mathrm{a}$ & 37.0 & $\mathrm{n}$ \\
\hline 516 & & 03 & 31 & 47.75 & -52 & 09 & 25.7 & 18.4 & 57996 & $\mathrm{a}$ & 104.0 & $\mathrm{n}$ \\
\hline 517 & & 03 & 31 & 50.81 & -52 & 01 & 26.1 & 18.1 & 22997 & $\mathrm{ae}$ & 65.4 & $\mathrm{y}$ \\
\hline 519 & CR161a & 03 & 31 & 52.56 & -52 & 18 & 26.0 & 17.3 & 18140 & $\mathrm{a}$ & 40.4 & $\mathrm{n}$ \\
\hline 520 & & 03 & 31 & 52.95 & -52 & 07 & 24.1 & 16.0 & 11765 & ae & 249.8 & $\mathrm{y}$ \\
\hline 522 & & 03 & 31 & 53.72 & -52 & 08 & 08.9 & 18.5 & 18624 & e & 105.0 & $\mathrm{y}$ \\
\hline 527 & & 03 & 31 & 57.79 & -52 & 41 & 13.0 & 18.1 & 16926 & $\mathrm{ae}$ & 86.5 & $\mathrm{y}$ \\
\hline 528 & E173 & 03 & 31 & 58.02 & -52 & 58 & 16.5 & 17.9 & 50096 & $\mathrm{a}$ & 599.6 & $\mathrm{n}$ \\
\hline 530 & & 03 & 32 & 04.30 & -53 & 13 & 14.1 & 17.4 & 17908 & e & 70.2 & $\mathrm{y}$ \\
\hline 532 & E174 & 03 & 32 & 07.51 & -52 & 15 & 39.0 & 16.7 & 18257 & e & 76.2 & y \\
\hline 533 & E175 & 03 & 32 & 07.80 & -52 & 33 & 29.8 & 17.5 & 11849 & e & 270.8 & $\mathrm{y}$ \\
\hline 534 & & 03 & 32 & 08.66 & -53 & 05 & 27.9 & 18.3 & 13705 & e & 62.1 & $\mathrm{y}$ \\
\hline 535 & & 03 & 32 & 09.70 & -53 & 29 & 58.1 & 17.9 & 32652 & $\mathrm{a}$ & 111.4 & $\mathrm{n}$ \\
\hline 537 & E181 & 03 & 32 & 12.52 & -52 & 56 & 42.4 & 17.0 & 18126 & e & 118.2 & $\mathrm{y}$ \\
\hline 538 & E178 & 03 & 32 & 12.57 & -52 & 43 & 49.7 & 17.2 & 17320 & $\mathrm{a}$ & 62.2 & $\mathrm{n}$ \\
\hline 540 & E182 & 03 & 32 & 14.40 & -52 & 35 & 51.1 & 16.8 & 16926 & $\mathrm{a}$ & 52.9 & $\mathrm{y}$ \\
\hline 541 & & 03 & 32 & 15.58 & -51 & 56 & 08.2 & 18.4 & -16 & $\mathrm{a}$ & 47.3 & $\mathrm{n}$ \\
\hline 544 & & 03 & 32 & 21.03 & -52 & 19 & 23.1 & 18.1 & 17987 & $\mathrm{ae}$ & 70.3 & $\mathrm{y}$ \\
\hline 547 & E185 & 03 & 32 & 26.74 & -52 & 22 & 53.6 & 16.7 & 16760 & $\mathrm{a}$ & 46.3 & $\mathrm{y}$ \\
\hline 548 & & 03 & 32 & 28.02 & -52 & 13 & 54.9 & 17.4 & 12479 & $\mathrm{a}$ & 103.9 & $\mathrm{y}$ \\
\hline 549 & & 03 & 32 & 28.24 & -53 & 09 & 20.2 & 17.8 & 33999 & e & 198.7 & $\mathrm{y}$ \\
\hline 550 & & 03 & 32 & 28.70 & -52 & 44 & 30.1 & 18.2 & 18125 & $\mathrm{a}$ & 29.1 & $\mathrm{n}$ \\
\hline
\end{tabular}


Table 2-Continued

\begin{tabular}{|c|c|c|c|c|c|c|c|c|c|c|c|c|}
\hline \multirow{2}{*}{$\frac{\text { ID }}{551}$} & \multirow[t]{2}{*}{ Other ID } & \multicolumn{3}{|c|}{$\alpha(\mathrm{J} 2000)$} & \multicolumn{3}{|c|}{$\delta(\mathrm{J} 2000)$} & \multirow{2}{*}{$\frac{\mathrm{B}_{J}}{17.4}$} & \multirow{2}{*}{$\frac{\mathrm{cz}}{16744}$} & \multirow{2}{*}{$\frac{\text { ref }}{\text { ae }}$} & \multirow{2}{*}{$\frac{\sigma_{V}}{27.5}$} & \multirow{2}{*}{$\frac{\text { Emission }}{\mathrm{y}}$} \\
\hline & & 03 & 32 & 29.48 & -52 & 30 & 18.4 & & & & & \\
\hline 552 & & 03 & 32 & 29.56 & -53 & 30 & 07.4 & 17.5 & 33018 & $\mathrm{ae}$ & 152.4 & $\mathrm{y}$ \\
\hline 553 & & 03 & 32 & 30.40 & -53 & 24 & 46.7 & 18.3 & 23307 & $\mathrm{a}$ & 69.3 & $\mathrm{y}$ \\
\hline 555 & & 03 & 32 & 30.70 & -52 & 01 & 20.0 & 17.2 & 18379 & e & 249.4 & $\mathrm{y}$ \\
\hline 558 & & 03 & 32 & 33.55 & -52 & 32 & 24.6 & 18.0 & 18692 & $\mathrm{ae}$ & 102.3 & $\mathrm{y}$ \\
\hline 559 & E188,CR153a & 03 & 32 & 34.66 & -52 & 21 & 16.9 & 17.2 & 18280 & $\mathrm{a}$ & 153.7 & $\mathrm{n}$ \\
\hline 560 & & 03 & 32 & 39.73 & -52 & 59 & 44.7 & 18.2 & 27763 & $\mathrm{a}$ & 94.4 & $\mathrm{n}$ \\
\hline 563 & & 03 & 32 & 42.41 & -52 & 17 & 55.4 & 18.5 & 18664 & e & 110.9 & $\mathrm{y}$ \\
\hline 565 & & 03 & 32 & 45.32 & -52 & 07 & 38.5 & 17.2 & 18395 & ae & 117.4 & $\mathrm{y}$ \\
\hline 566 & & 03 & 32 & 45.40 & -52 & 10 & 57.7 & 18.1 & 20243 & e & 117.8 & $\mathrm{y}$ \\
\hline 567 & & 03 & 32 & 45.92 & -52 & 33 & 31.0 & 16.7 & -9 & $\mathrm{a}$ & 17.9 & $\mathrm{n}$ \\
\hline 569 & & 03 & 32 & 47.45 & -53 & 25 & 50.7 & 16.1 & 22775 & $\mathrm{a}$ & 81.5 & $\mathrm{n}$ \\
\hline 570 & & 03 & 32 & 47.49 & -53 & 28 & 24.6 & 17.9 & 23822 & ae & 145.9 & $\mathrm{y}$ \\
\hline 572 & & 03 & 32 & 48.41 & -52 & 51 & 50.3 & 18.4 & 33 & $\mathrm{a}$ & 18.1 & $\mathrm{n}$ \\
\hline 574 & & 03 & 32 & 50.67 & -53 & 27 & 37.3 & 17.6 & 22548 & $\mathrm{a}$ & 59.5 & $\mathrm{n}$ \\
\hline 576 & & 03 & 32 & 55.39 & -52 & 47 & 56.8 & 17.2 & 12752 & ae & 150.8 & $\mathrm{y}$ \\
\hline 578 & & 03 & 32 & 57.35 & -52 & 16 & 25.2 & 18.0 & -33 & $\mathrm{a}$ & 278.2 & $\mathrm{n}$ \\
\hline 579 & & 03 & 32 & 57.65 & -52 & 54 & 05.2 & 18.2 & 27779 & $\mathrm{ae}$ & 152.6 & $\mathrm{y}$ \\
\hline 581 & & 03 & 32 & 58.46 & -52 & 15 & 16.2 & 18.5 & 38932 & $\mathrm{ae}$ & 110.6 & $\mathrm{y}$ \\
\hline 582 & & 03 & 32 & 59.93 & -53 & 03 & 31.7 & 16.1 & 11796 & $\mathrm{ae}$ & 131.8 & $\mathrm{y}$ \\
\hline 583 & & 03 & 33 & 10.22 & -53 & 29 & 27.9 & 17.7 & 22533 & $\mathrm{a}$ & 221.6 & $\mathrm{n}$ \\
\hline 587 & & 03 & 33 & 17.61 & -52 & 12 & 41.2 & 17.2 & -49 & $\mathrm{a}$ & 285.7 & $\mathrm{n}$ \\
\hline 588 & E191 & 03 & 33 & 18.61 & -52 & 27 & 59.7 & 17.2 & 17676 & $\mathrm{a}$ & 31.7 & $\mathrm{n}$ \\
\hline 589 & & 03 & 33 & 18.94 & -53 & 28 & 44.9 & 17.6 & 22761 & $\mathrm{a}$ & 46.6 & $\mathrm{n}$ \\
\hline 590 & & 03 & 33 & 19.22 & -53 & 31 & 36.4 & 17.8 & 23651 & $\mathrm{a}$ & 63.6 & $\mathrm{n}$ \\
\hline 591 & E192 & 03 & 33 & 20.12 & -52 & 28 & 43.6 & 17.5 & 17569 & $\mathrm{ae}$ & 38.6 & $\mathrm{y}$ \\
\hline 592 & & 03 & 33 & 20.40 & -52 & 01 & 59.2 & 17.5 & 25216 & $\mathrm{ae}$ & 60.5 & $\mathrm{y}$ \\
\hline 593 & & 03 & 33 & 21.83 & -52 & 33 & 09.2 & 16.2 & 13405 & ae & 412.7 & $\mathrm{y}$ \\
\hline 595 & E193 & 03 & 33 & 24.92 & -52 & 30 & 24.9 & 17.5 & 17572 & $\mathrm{ae}$ & 98.7 & $\mathrm{y}$ \\
\hline 596 & & 03 & 33 & 25.94 & -52 & 50 & 12.7 & 18.4 & 23014 & $\mathrm{a}$ & 67.5 & $\mathrm{n}$ \\
\hline 597 & & 03 & 33 & 28.27 & -53 & 13 & 17.5 & 18.2 & 17446 & e & 248.3 & $\mathrm{y}$ \\
\hline 598 & & 03 & 33 & 30.06 & -53 & 10 & 23.9 & 16.2 & 17052 & $\mathrm{ae}$ & 273.0 & $\mathrm{y}$ \\
\hline 599 & & 03 & 33 & 34.79 & -53 & 12 & 24.5 & 18.3 & 18453 & e & 102.9 & $\mathrm{y}$ \\
\hline
\end{tabular}


Table 2-Continued

\begin{tabular}{|c|c|c|c|c|c|c|c|c|c|c|c|c|}
\hline \multirow{2}{*}{$\frac{\text { ID }}{602}$} & \multirow{2}{*}{ Other ID } & \multicolumn{3}{|c|}{$\alpha(\mathrm{J} 2000)$} & \multicolumn{3}{|c|}{$\delta(\mathrm{J} 2000)$} & \multirow{2}{*}{$\frac{\mathrm{B}_{J}}{18.5}$} & \multirow{2}{*}{$\frac{\mathrm{cz}}{53569}$} & \multirow{2}{*}{$\frac{\text { ref }}{\mathrm{e}}$} & \multirow{2}{*}{$\frac{\sigma_{V}}{161.5}$} & \multirow{2}{*}{$\begin{array}{c}\text { Emission } \\
\mathrm{y}\end{array}$} \\
\hline & & 03 & 33 & 37.63 & -52 & 16 & 25.0 & & & & & \\
\hline 603 & & 03 & 33 & 38.88 & -52 & 12 & 02.7 & 16.6 & 20306 & $\mathrm{a}$ & 158.7 & $\mathrm{n}$ \\
\hline 604 & & 03 & 33 & 45.13 & -52 & 19 & 31.1 & 18.4 & 20333 & $\mathrm{ae}$ & 117.7 & $\mathrm{y}$ \\
\hline 605 & & 03 & 33 & 46.58 & -52 & 26 & 42.6 & 17.6 & 31943 & $\mathrm{ae}$ & 101.4 & y \\
\hline 606 & & 03 & 33 & 49.97 & -53 & 01 & 34.4 & 18.3 & 17703 & e & 185.4 & $\mathrm{y}$ \\
\hline 607 & & 03 & 33 & 52.74 & -52 & 14 & 00.4 & 17.8 & 19721 & e & 354.1 & $\mathrm{y}$ \\
\hline 608 & & 03 & 33 & 53.05 & -52 & 11 & 07.5 & 17.2 & 19983 & $\mathrm{ae}$ & 70.2 & $\mathrm{y}$ \\
\hline 611 & & 03 & 33 & 59.66 & -52 & 10 & 10.0 & 17.4 & 44705 & $\mathrm{a}$ & 83.6 & $\mathrm{n}$ \\
\hline 612 & & 03 & 34 & 03.19 & -52 & 10 & 58.2 & 17.6 & 20734 & $\mathrm{a}$ & 91.6 & $\mathrm{y}$ \\
\hline 614 & & 03 & 34 & 11.99 & -53 & 23 & 42.1 & 17.9 & 18067 & $\mathrm{a}$ & 68.2 & $\mathrm{n}$ \\
\hline 615 & & 03 & 34 & 15.31 & -52 & 43 & 38.9 & 18.4 & 31348 & $\mathrm{ae}$ & 58.8 & y \\
\hline 616 & & 03 & 34 & 18.43 & -53 & 07 & 32.6 & 17.7 & 20429 & $\mathrm{a}$ & 57.6 & $\mathrm{y}$ \\
\hline 617 & & 03 & 34 & 18.65 & -53 & 11 & 44.9 & 18.3 & 16953 & e & 46.5 & $\mathrm{y}$ \\
\hline 618 & & 03 & 34 & 21.89 & -53 & 02 & 49.8 & 17.9 & 21710 & $\mathrm{a}$ & 54.0 & $\mathrm{n}$ \\
\hline 619 & & 03 & 34 & 24.84 & -52 & 25 & 48.3 & 18.2 & 19409 & e & 85.0 & y \\
\hline 620 & & 03 & 34 & 28.25 & -52 & 33 & 41.7 & 17.1 & 13530 & e & 223.9 & y \\
\hline 622 & & 03 & 34 & 29.79 & -53 & 00 & 16.1 & 18.4 & 44485 & e & 184.7 & $\mathrm{y}$ \\
\hline 623 & & 03 & 34 & 33.19 & -52 & 41 & 00.8 & 17.6 & 11553 & $\mathrm{ae}$ & 63.9 & y \\
\hline 625 & & 03 & 34 & 37.17 & -53 & 26 & 52.4 & 17.8 & 18479 & $\mathrm{a}$ & 78.6 & $\mathrm{n}$ \\
\hline 626 & & 03 & 34 & 39.77 & -52 & 29 & 40.9 & 17.8 & 22790 & $\mathrm{a}$ & 76.0 & $\mathrm{n}$ \\
\hline 627 & & 03 & 34 & 43.14 & -52 & 50 & 00.5 & 18.4 & 44417 & e & 241.1 & $\mathrm{y}$ \\
\hline 628 & & 03 & 34 & 47.64 & -52 & 58 & 30.5 & 17.5 & 20339 & $\mathrm{a}$ & 78.7 & $\mathrm{y}$ \\
\hline 629 & & 03 & 34 & 49.29 & -53 & 14 & 13.7 & 16.7 & 9245 & $\mathrm{ae}$ & 298.8 & $\mathrm{y}$ \\
\hline 635 & & 03 & 35 & 07.67 & -53 & 00 & 54.1 & 16.1 & 17371 & $\mathrm{a}$ & 91.0 & $\mathrm{y}$ \\
\hline 636 & & 03 & 35 & 11.31 & -52 & 50 & 59.5 & 18.1 & 17231 & $\mathrm{ae}$ & 129.6 & $\mathrm{y}$ \\
\hline 637 & & 03 & 35 & 17.70 & -52 & 59 & 25.2 & 17.5 & 20927 & ae & 47.6 & $\mathrm{y}$ \\
\hline 638 & & 03 & 35 & 26.80 & -53 & 08 & 51.0 & 16.4 & 16939 & $\mathrm{ae}$ & 80.5 & $\mathrm{y}$ \\
\hline 640 & & 03 & 35 & 34.68 & -52 & 53 & 23.1 & 16.6 & 22990 & $\mathrm{a}$ & 66.5 & $\mathrm{n}$ \\
\hline
\end{tabular}


Table 3. Groups and Filaments in A3125/A3128

\begin{tabular}{|c|c|c|c|c|c|c|c|c|}
\hline (1) & $(2)$ & (3) & & (5) & (6) & $(7)$ & & (9) \\
\hline Group ID & $n$ & $c z$ Limits & $\alpha$ Limits & $\delta$ Limits & K-S Test & $\alpha-c z$ Corr. & $\delta-c z$ Corr. & $\sigma_{\alpha}$ \\
\hline G1 & 25 & $18600-19400$ & $-18.0-10.0$ & $\ldots$ & $4.4 \times 10^{-3}$ & 0.54 & 0.40 & 6.6 \\
\hline (Control) & 24 & $18600-19400$ & $\cdots$ & $\cdots$ & $\cdots$ & 0.91 & 0.92 & 17.1 \\
\hline G2 & $14^{\mathrm{a}}$ & $18200-19100$ & $\ldots$ & $-80 .--50$ & $7.9 \times 10^{-11}$ & 0.78 & 0.14 & 9.5 \\
\hline (Control) & 83 & $18200-19100$ & $\ldots$ & $\ldots$ & $\ldots$ & 0.41 & 0.97 & 20.1 \\
\hline $\mathrm{F} 1$ & 19 & $19400-20500$ & $\ldots$ & $-10.0-22.0$ & 0.20 & 0.018 & $1.13 \times 10^{-3}$ & 26.0 \\
\hline (Control) & 14 & $19400-20500$ & $\ldots$ & $\ldots$ & $\ldots$ & $1.69 \times 10^{-3}$ & 0.59 & 27.7 \\
\hline $\mathrm{F} 2$ & 10 & $19100-20000$ & $\ldots$ & $-53.0--22.0$ & 0.097 & 0.19 & $1.75 \times 10^{-3}$ & 20.7 \\
\hline (Control) & 25 & $19100-20000$ & $\cdots$ & $\cdots$ & $\cdots$ & 0.024 & 0.13 & 22.2 \\
\hline $\mathrm{C} 1$ & 24 & $17500-18300$ & $-30.0--22.0$ & $\ldots$ & 0.025 & 0.94 & 0.91 & 21.5 \\
\hline (Control) & 118 & $17500-18300$ & . & $\ldots$ & $\ldots$ & 1.00 & 1.00 & 24.2 \\
\hline
\end{tabular}

${ }^{\text {a } O n e ~ g a l a x y ~ w i t h ~ d i s c r e p a n t ~ R A ~ h a s ~ b e e n ~ e x c l u d e d ~ f r o m ~ t h e ~ o r i g i n a l ~ g r o u p ~ o f ~} 15$ galaxies. 
Table 4. Small Groups and Filaments in A3125/A3128

\begin{tabular}{lccccrr}
\hline \hline Group ID & $n$ & $c z$ Limits & $\alpha$ Limits & $\delta$ Limits & $\sigma_{\alpha}$ & $\sigma_{\delta}$ \\
\hline G3 & 4 & $19700-20100$ & $\ldots$ & $-18.0--7.0$ & 4.0 & 2.2 \\
(Control) & 9 & $19700-20100$ & $\ldots$ & $\ldots$ & 24.8 & 26.5 \\
G4 & 5 & $21500-22400$ & $\ldots$ & $-20.0-5.0$ & 7.1 & 6.3 \\
(Control) & 4 & $21500-22400$ & $\ldots$ & $\ldots$ & 27.5 & 22.0 \\
G5 & 5 & $20300-21800$ & $-2.0-7.0$ & $\ldots$ & 1.5 & 18.7 \\
$\ldots$ a & 4 & $20300-21800$ & $-2.0-7.0$ & $\ldots$ & 1.6 & 1.9 \\
(Control) & 12 & $20300-21800$ & $\ldots$ & $\ldots$ & 27.0 & 32.5 \\
G6 & 5 & $15000-16300$ & $\ldots$ & $-80 .--60$. & 11.0 & 2.9 \\
(Control) & 4 & $15000-16300$ & $\ldots$ & $\ldots$ & 7.0 & 4.4 \\
G7 & 8 & $22000-24100$ & $12.0-30.0$ & $\ldots$ & 3.0 & 13.5 \\
$\ldots$ a & 7 & $22000-24100$ & $12.0-30.0$ & $\ldots$ & 2.8 & 2.3 \\
(Control) & 41 & $22000-24100$ & $\ldots$ & $\ldots$ & 27.4 & 21.0 \\
& & & & & & \\
\hline
\end{tabular}

${ }^{a}$ One galaxy with discrepant Dec has been excluded from the original group.

Table 5. Temperature and Abundance Data for the ICM in A3128

\begin{tabular}{|c|c|c|c|c|}
\hline Region & Radius & $\mathrm{kT}(\mathrm{keV})$ & $\mathrm{A}$ & Reduced $\chi^{2}$ \\
\hline NE (Global) & $0 "-180 "$ & $3.9_{-0.6}^{+0.7}$ & $0.13_{0.0}^{0.3}$ & 1.08 \\
\hline NE (Small Core) & $0 "-22.5 "$ & $5.1+2.7$ & 0.935 .0 & 0.99 \\
\hline NE (Large Core) & $0 "-45 "$ & $5.2+2.3$ & $0.18 \begin{array}{l}0.7 \\
0.0\end{array}$ & 0.72 \\
\hline NE (Outer Region) & $45 "-90 "$ & $3.9+1.3$ & 0.170 .6 & 1.15 \\
\hline NE (Outer Region) & $90 "-180 "$ & $3.6_{-0.6}^{+1.0}$ & $0.13 \begin{array}{l}0.4 \\
0.0\end{array}$ & 1.29 \\
\hline SW (Large Core) & $0 "-90 "$ & $3.3_{-0.4}^{+0.4}$ & $0.61_{0.9}^{0.9}$ & 1.29 \\
\hline SW (Outer Region) & $90 "-180 "$ & $3.7_{-0.5}^{+0.4}$ & $0.34 \begin{array}{l}0.4 \\
0.6 \\
0.2\end{array}$ & 0.99 \\
\hline
\end{tabular}




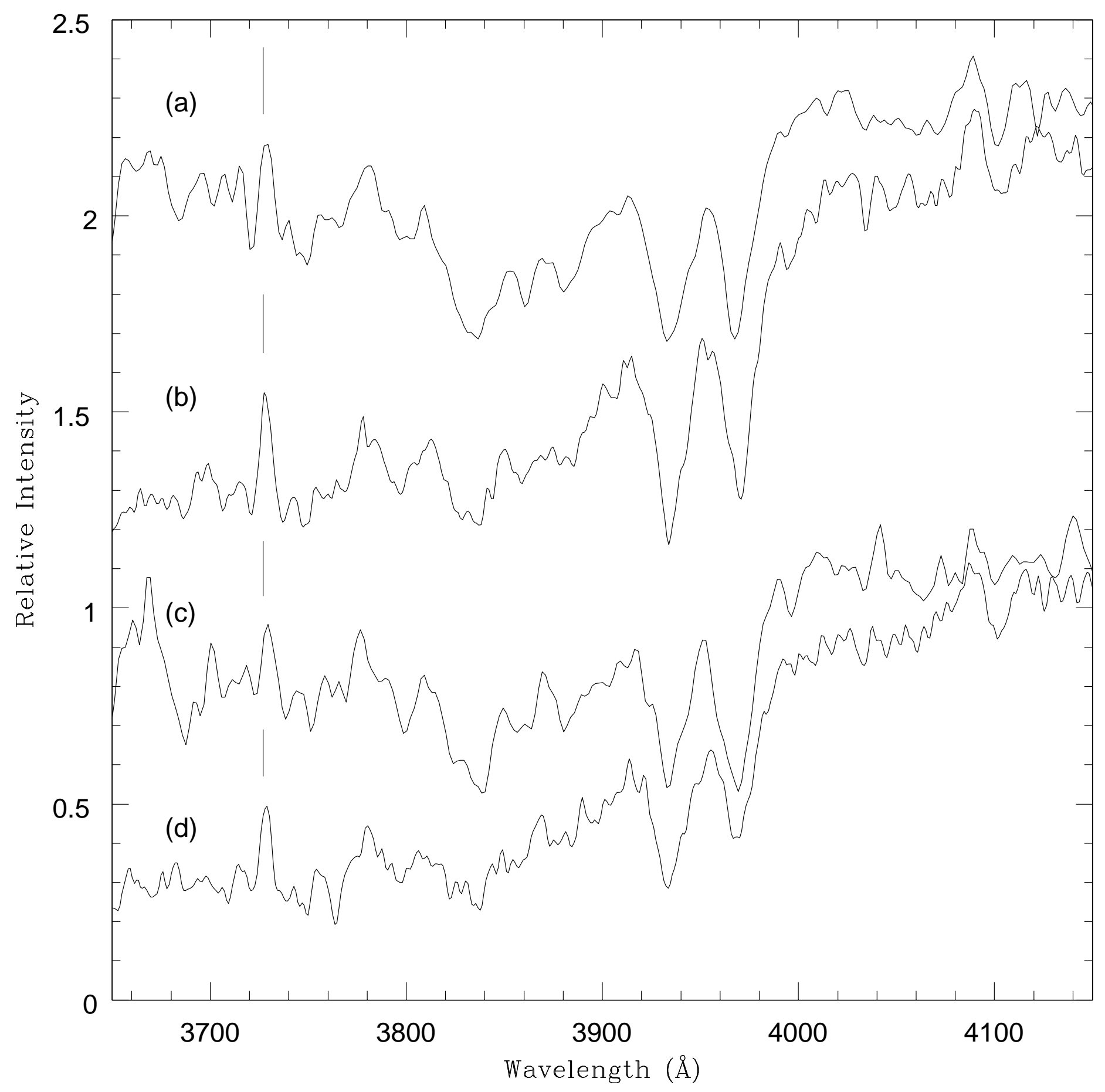




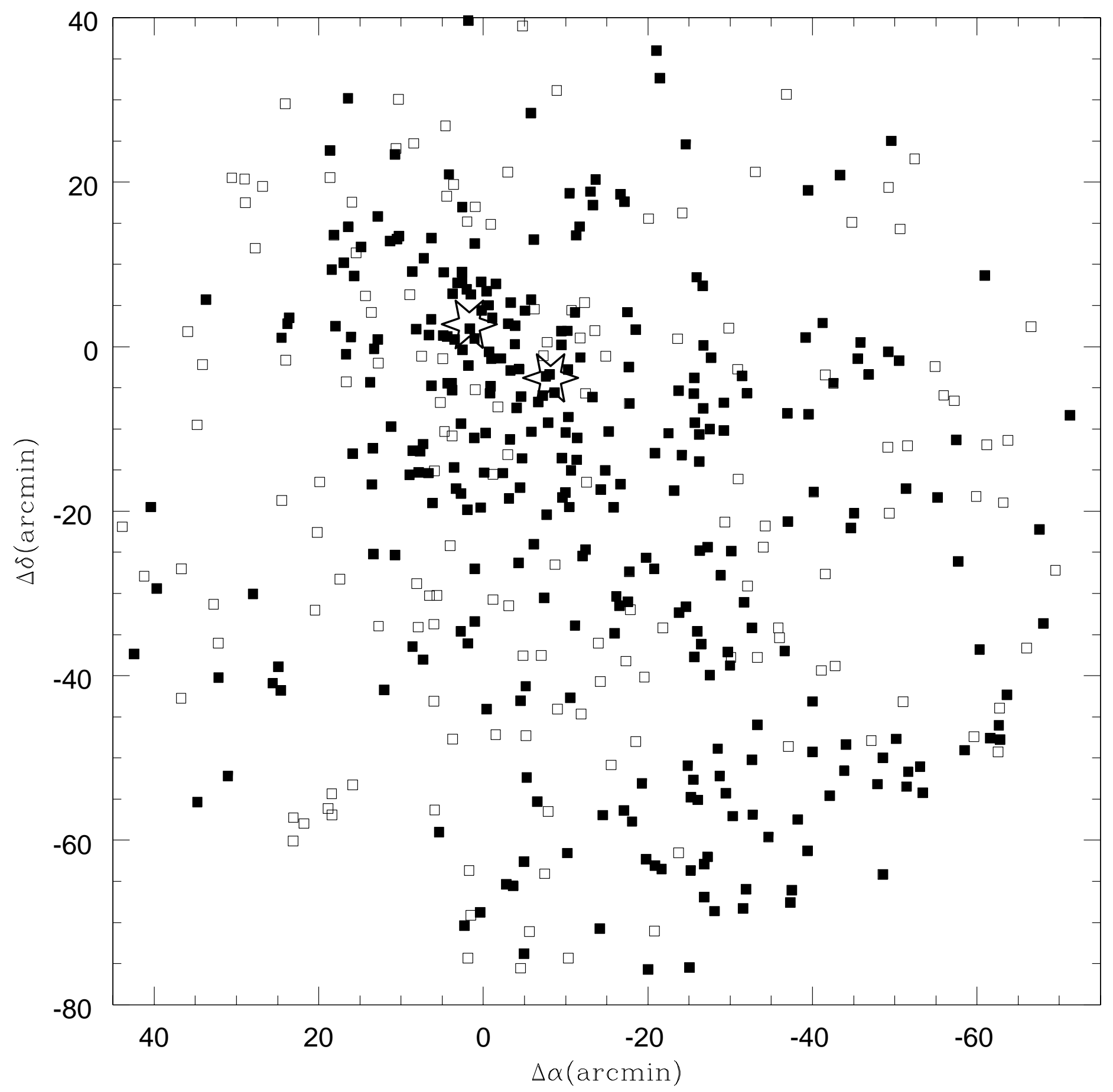




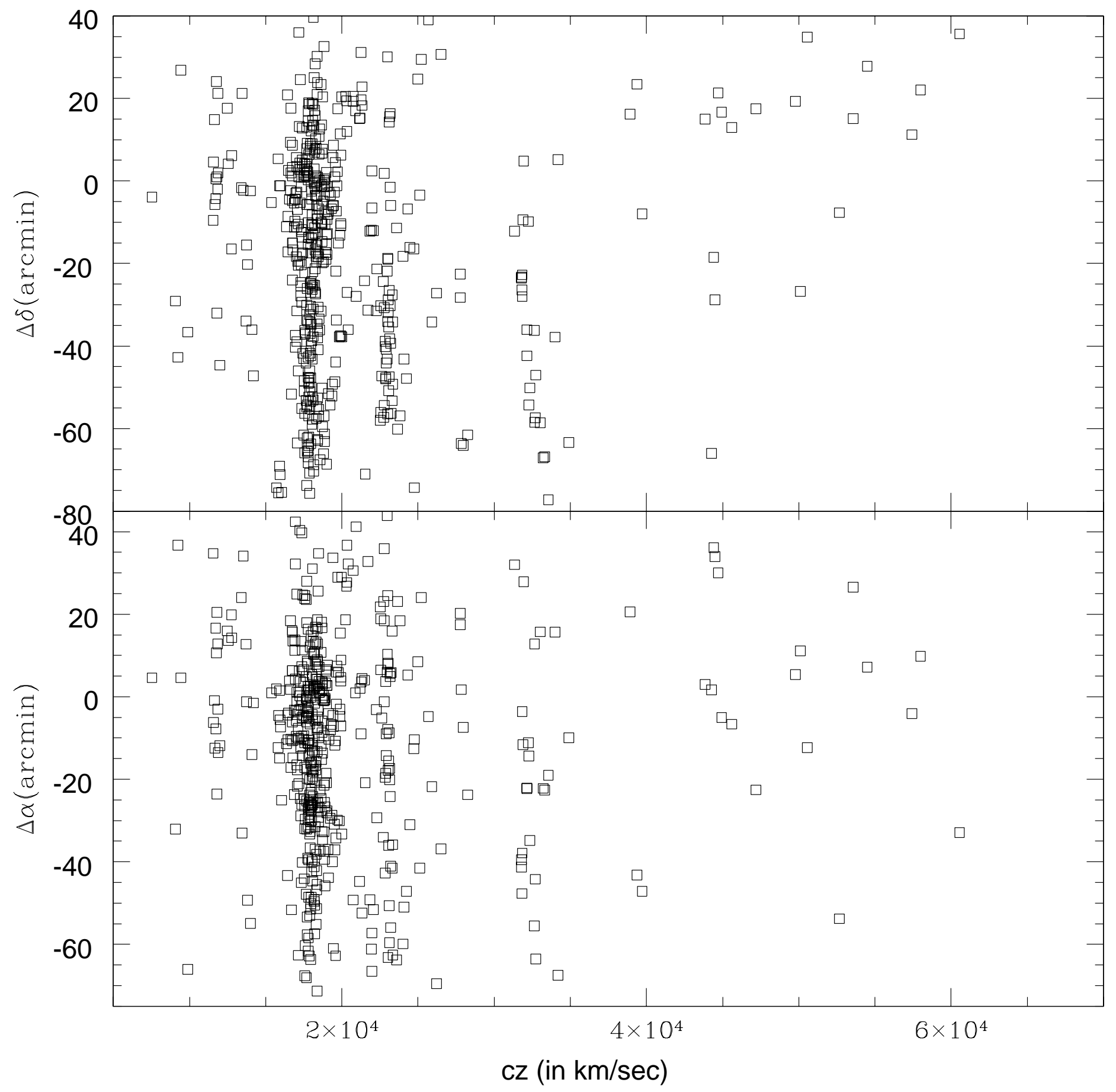




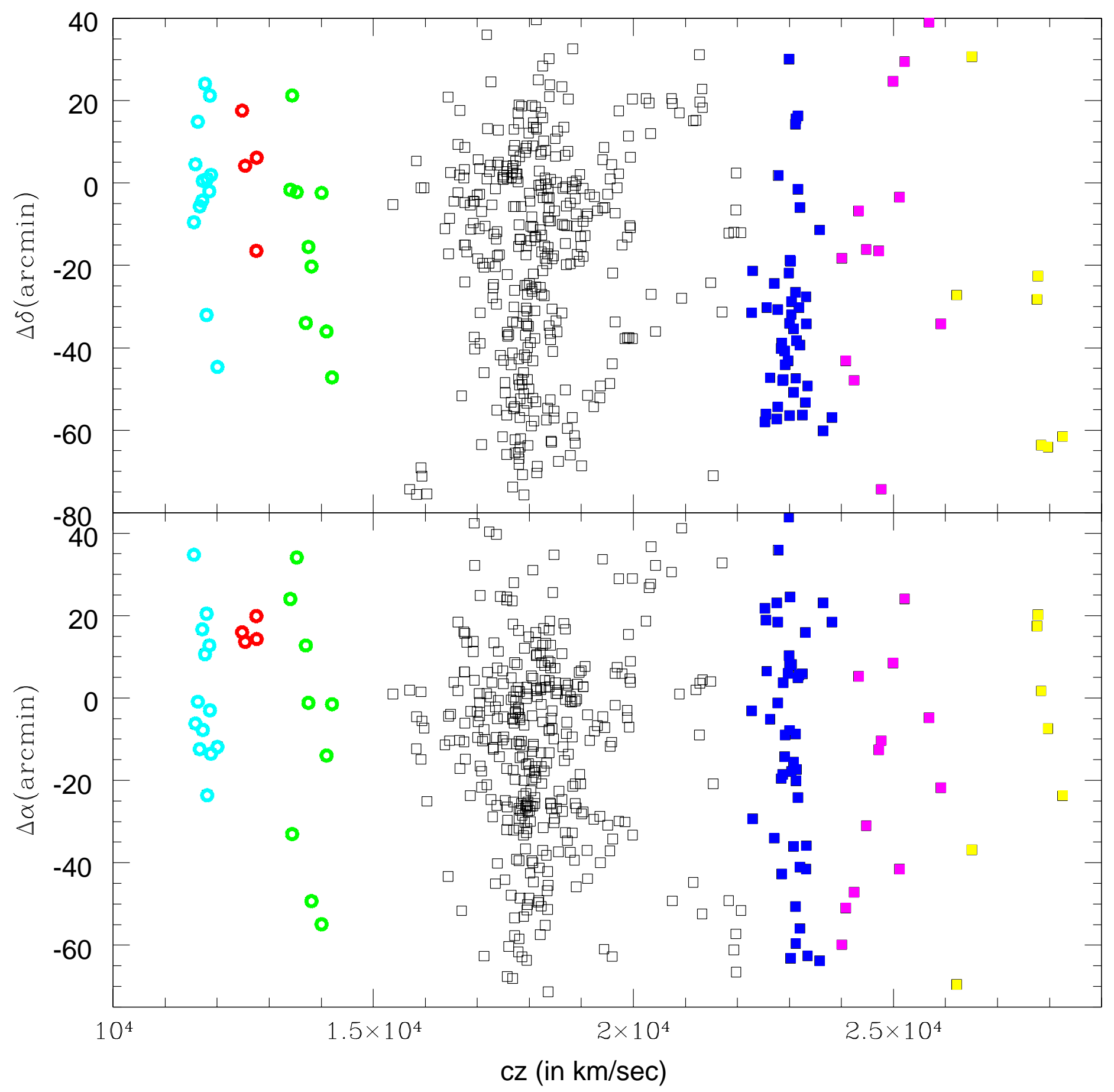




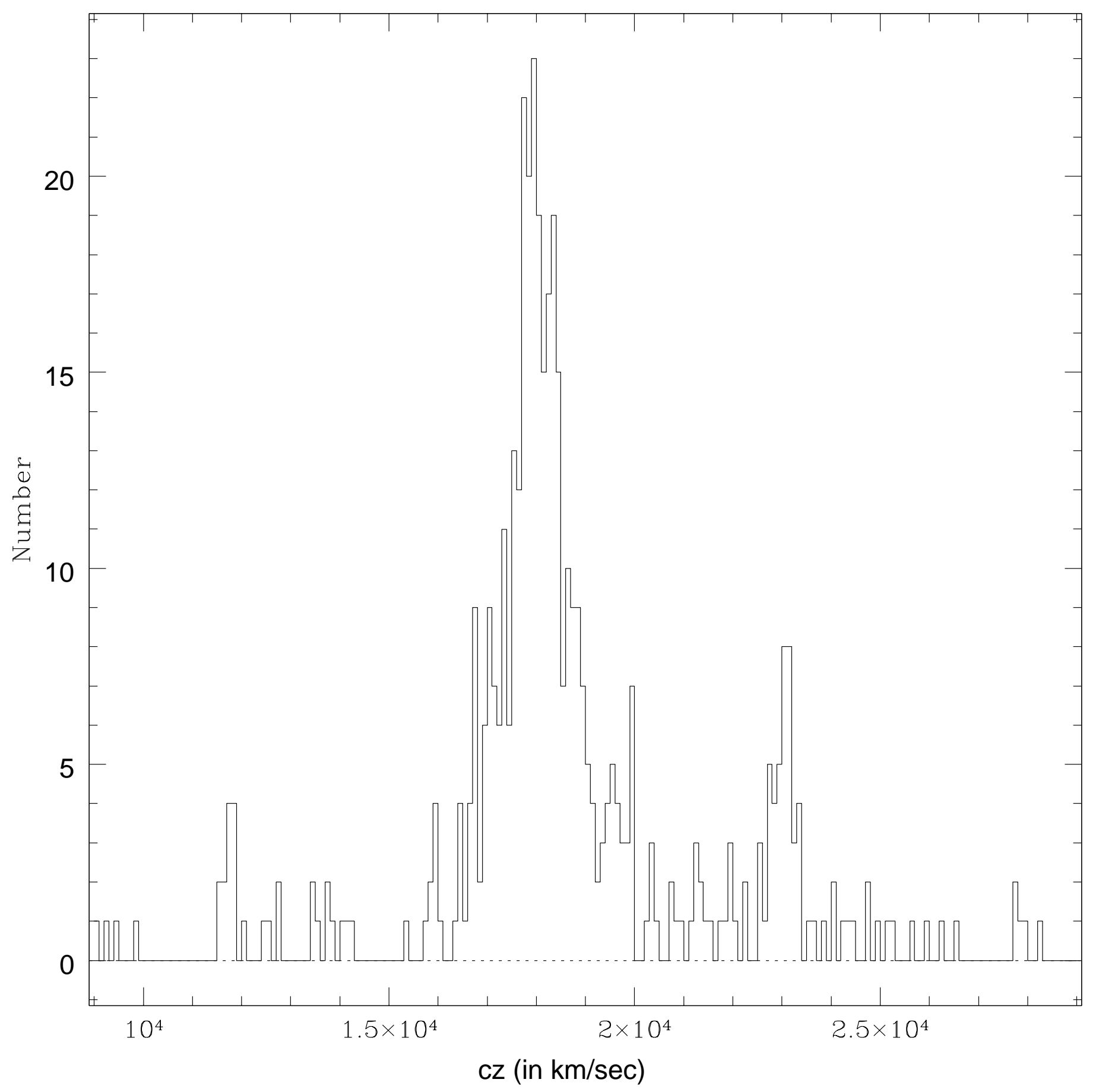




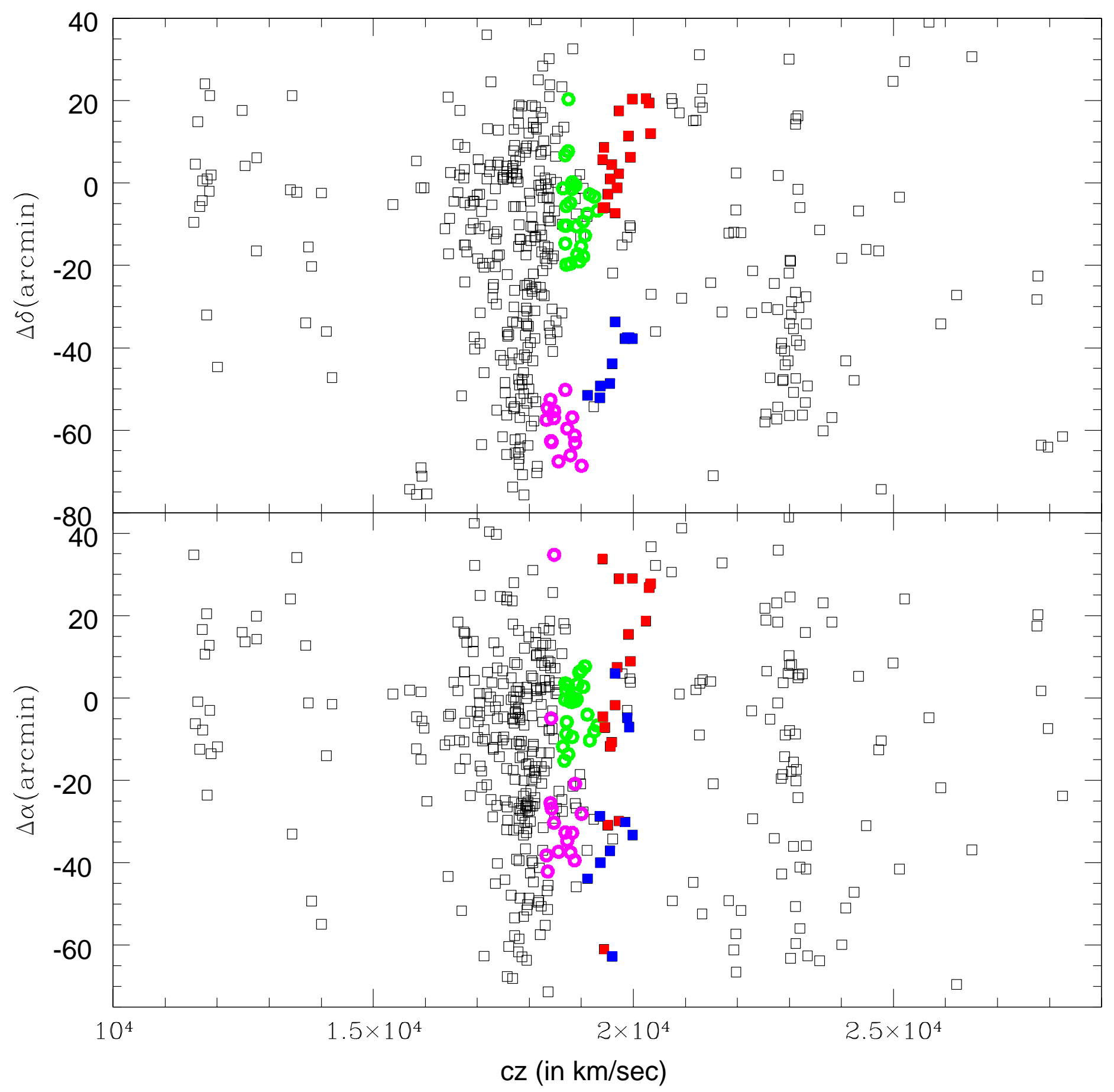




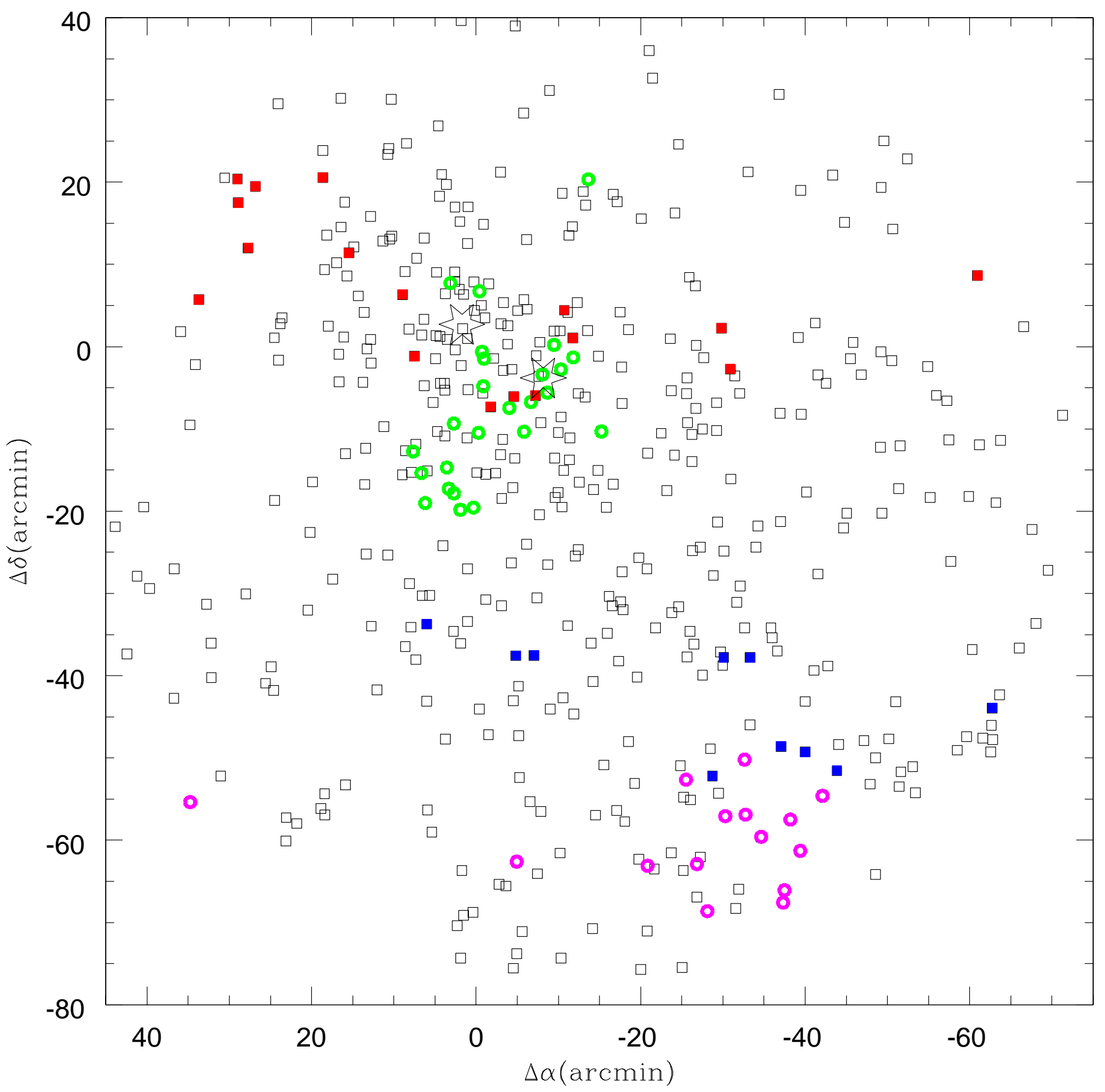




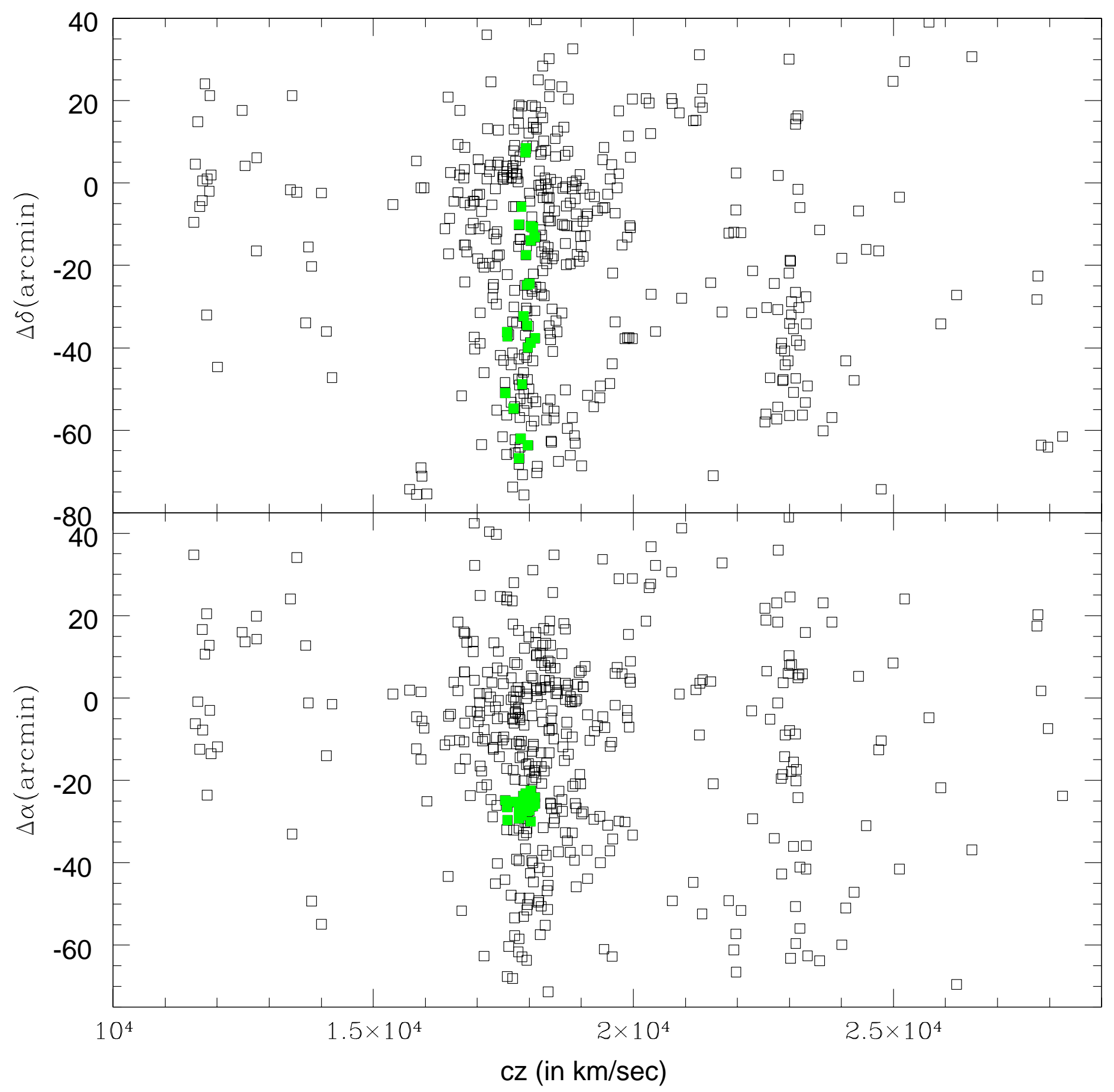




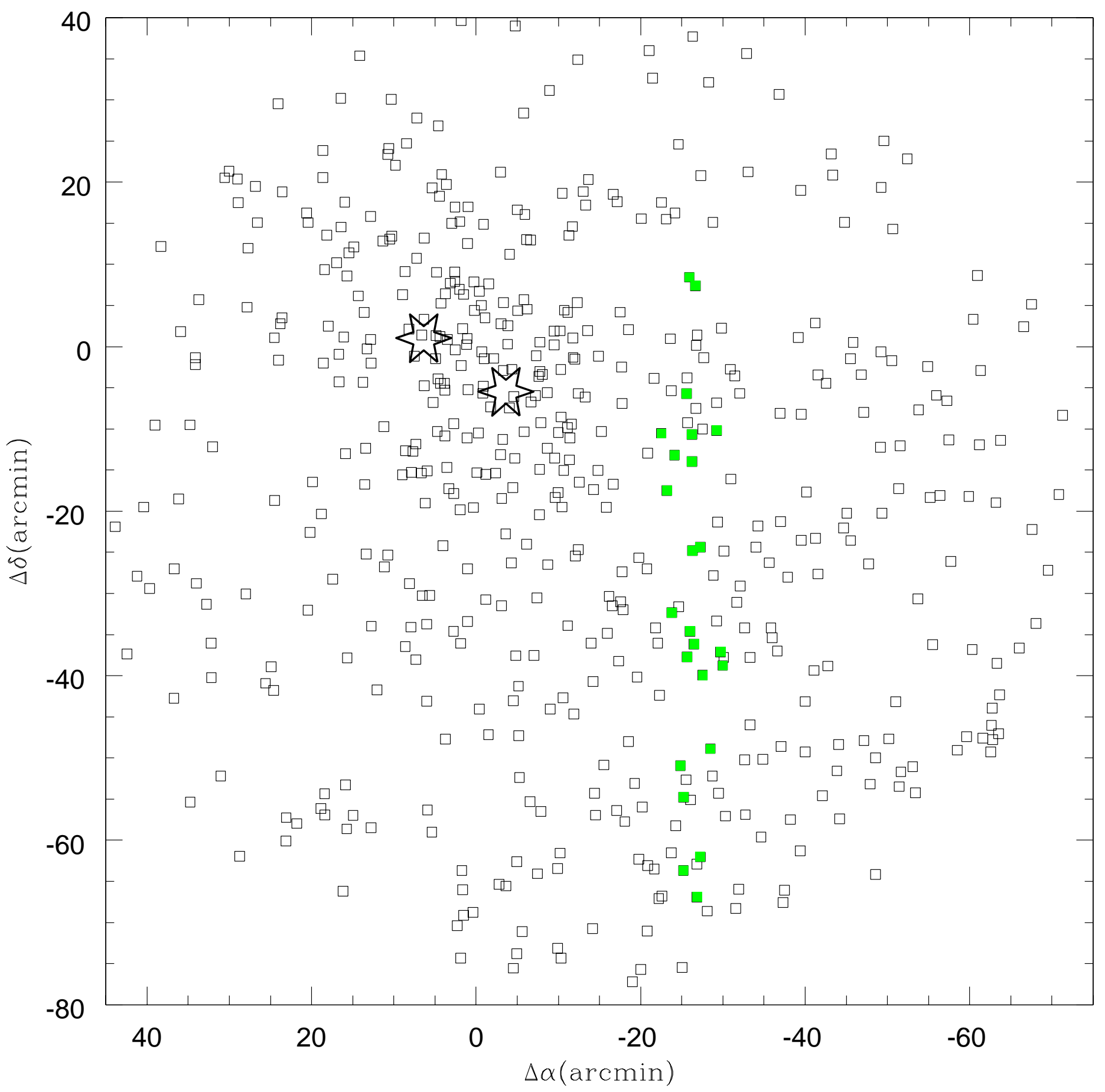




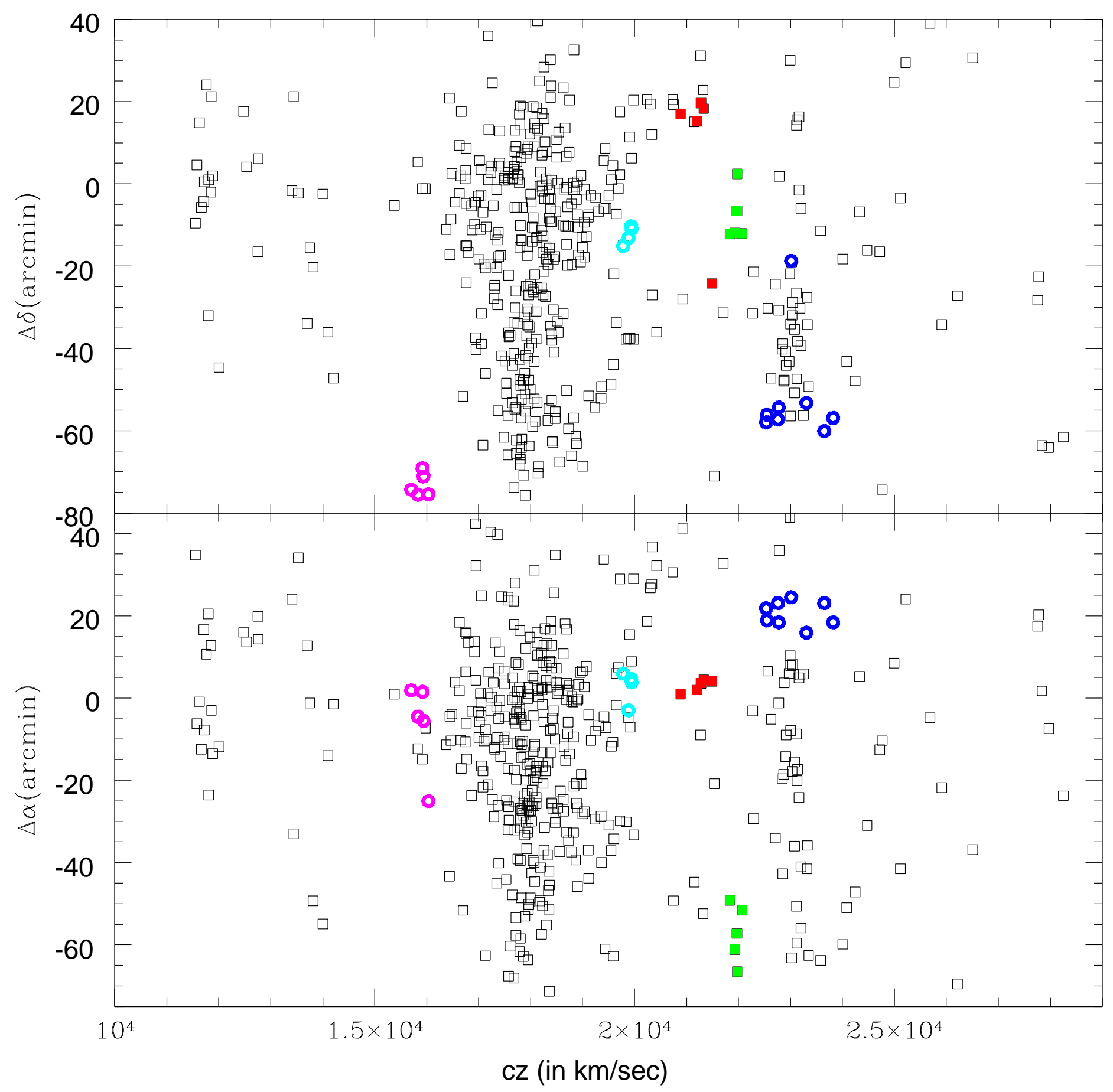




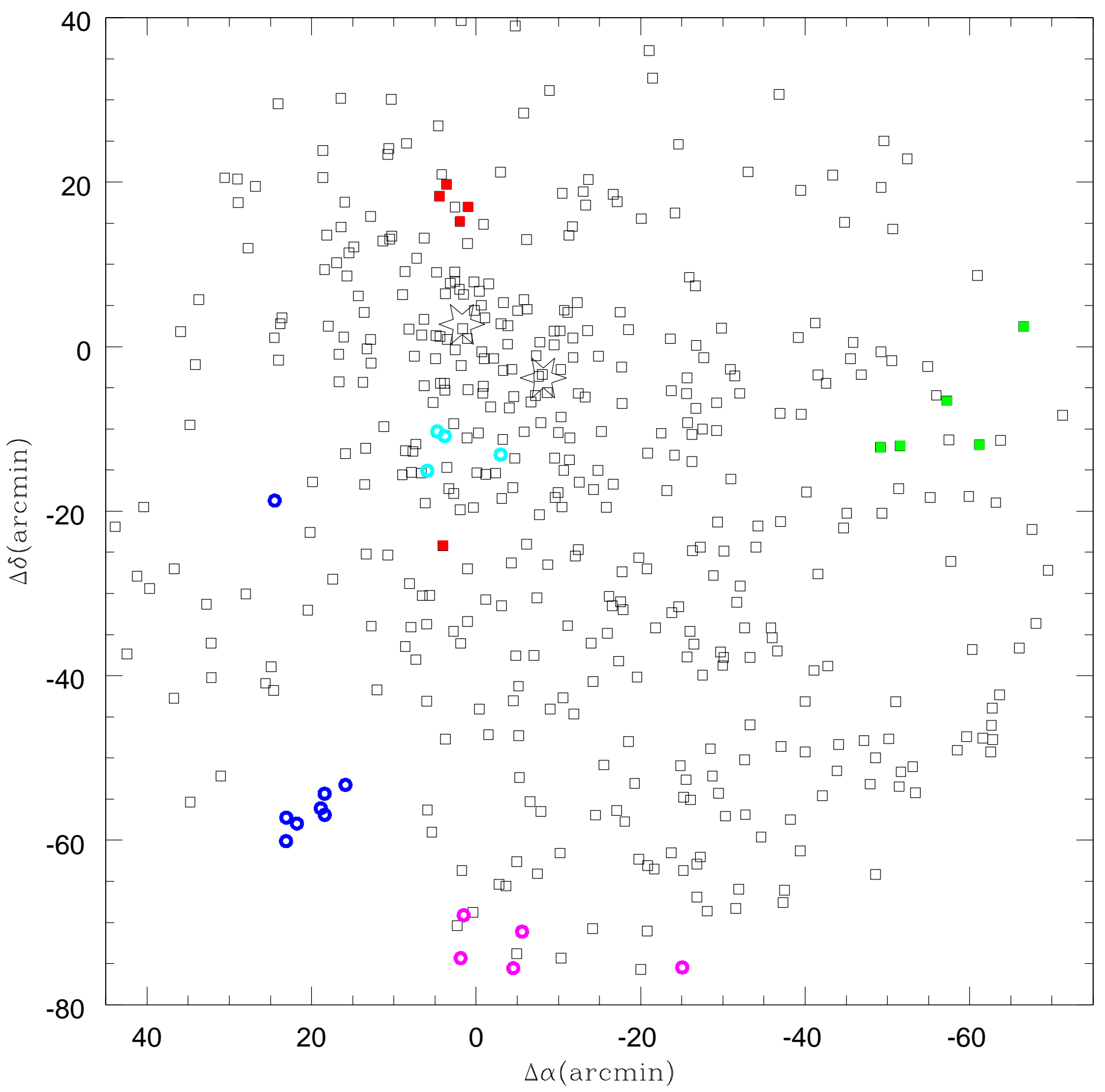




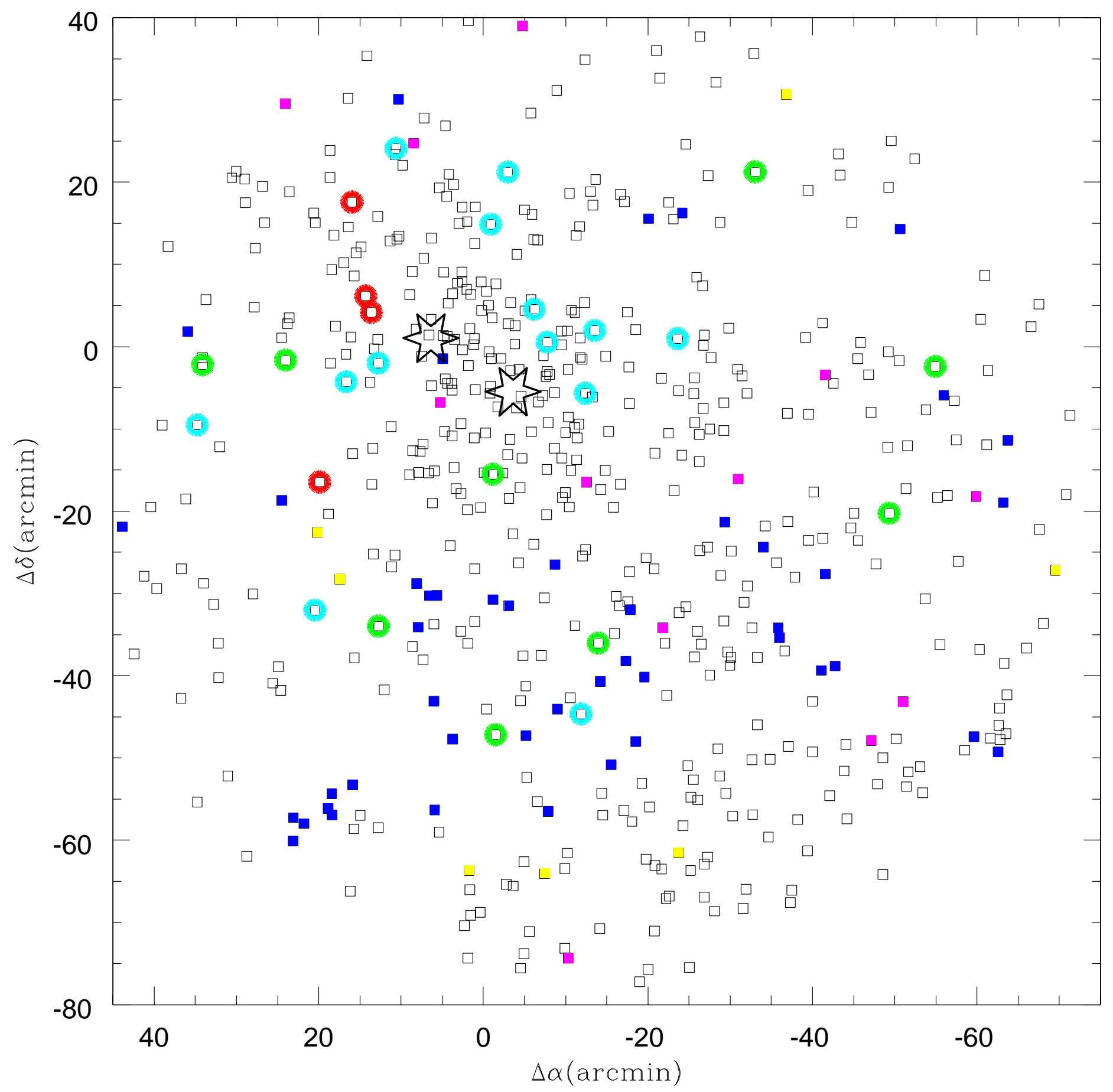




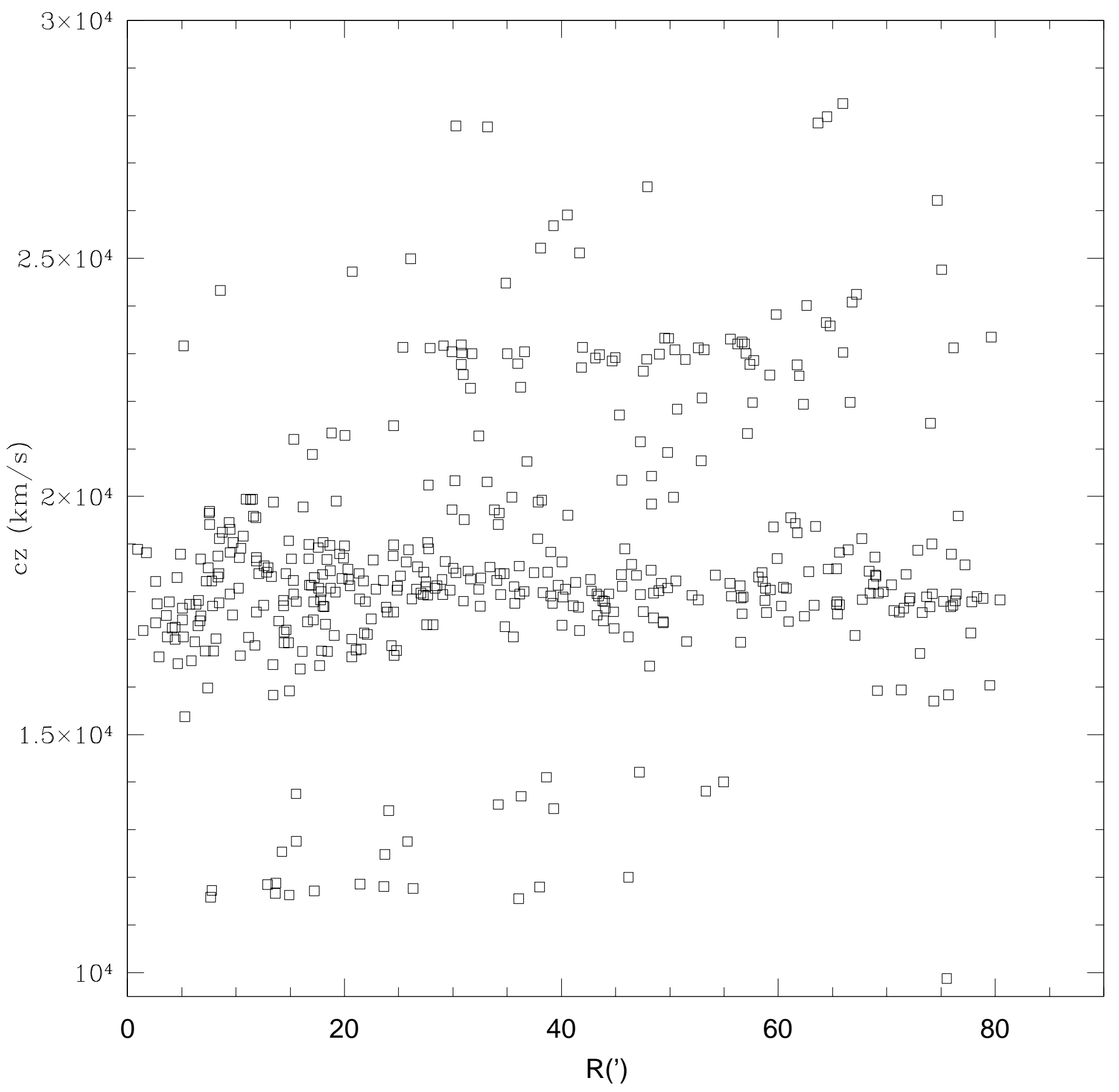




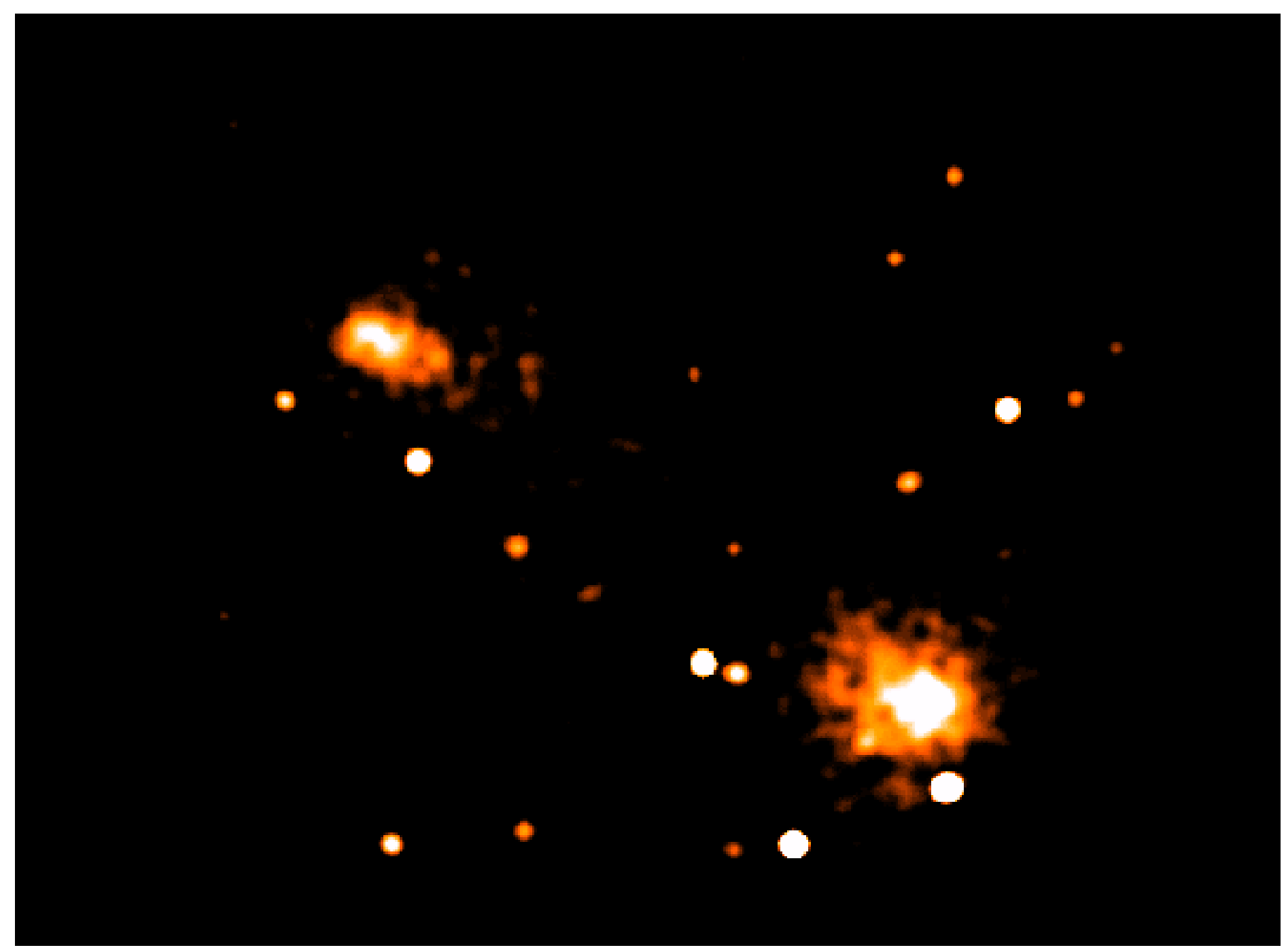




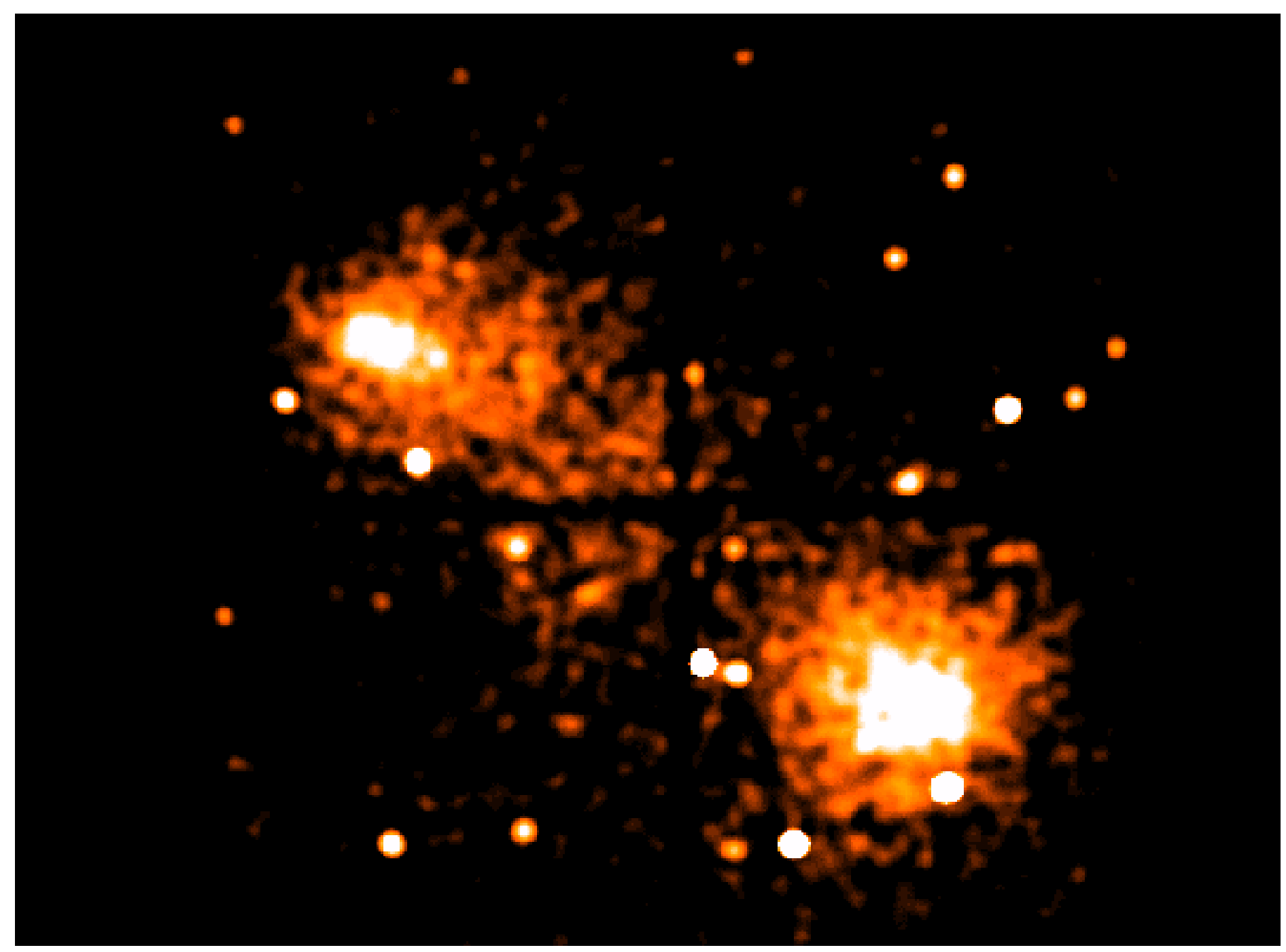




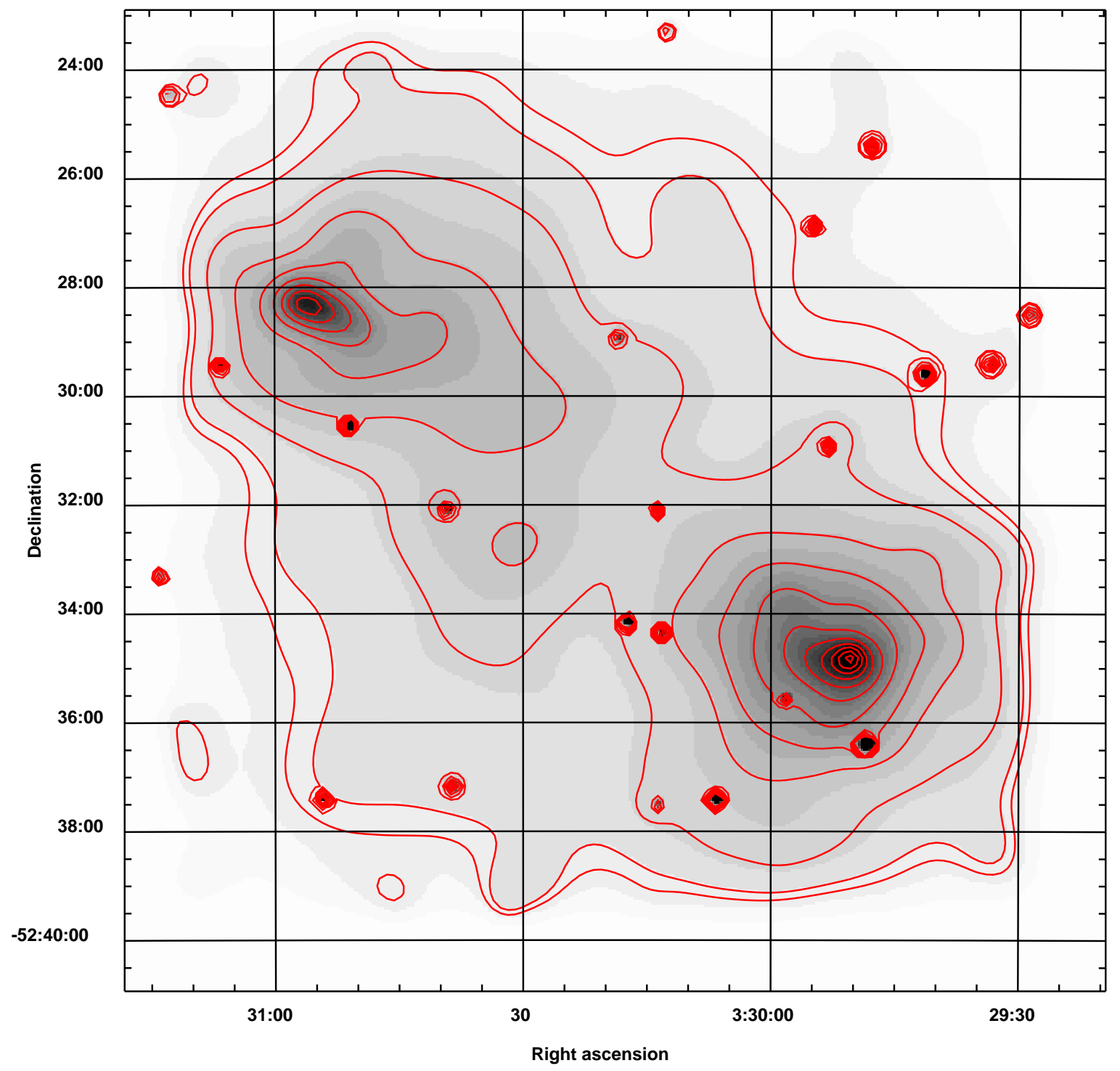


This figure "Rose.fig17.jpg" is available in "jpg" format from: http://arxiv.org/ps/astro-ph/0112346v1 


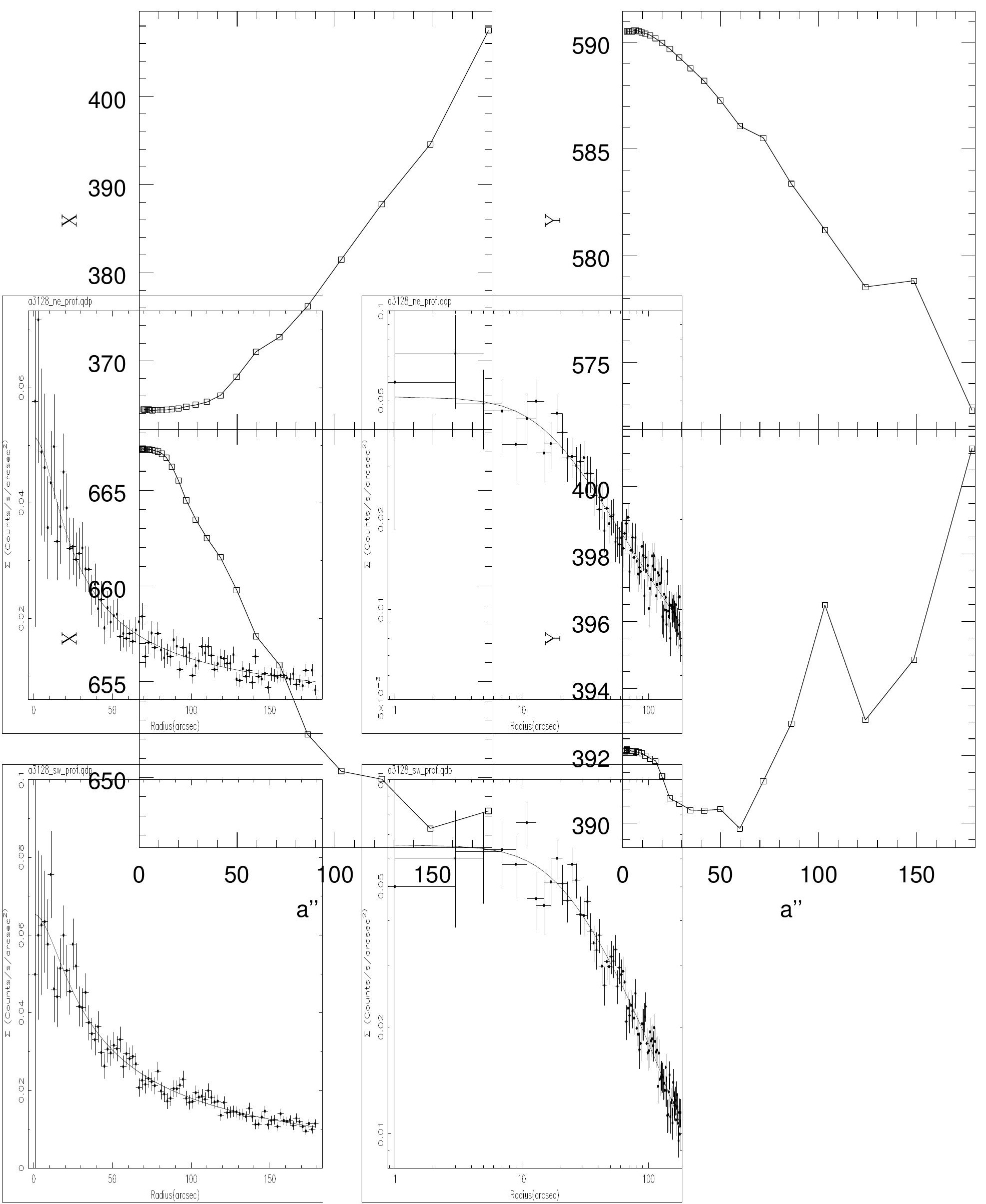



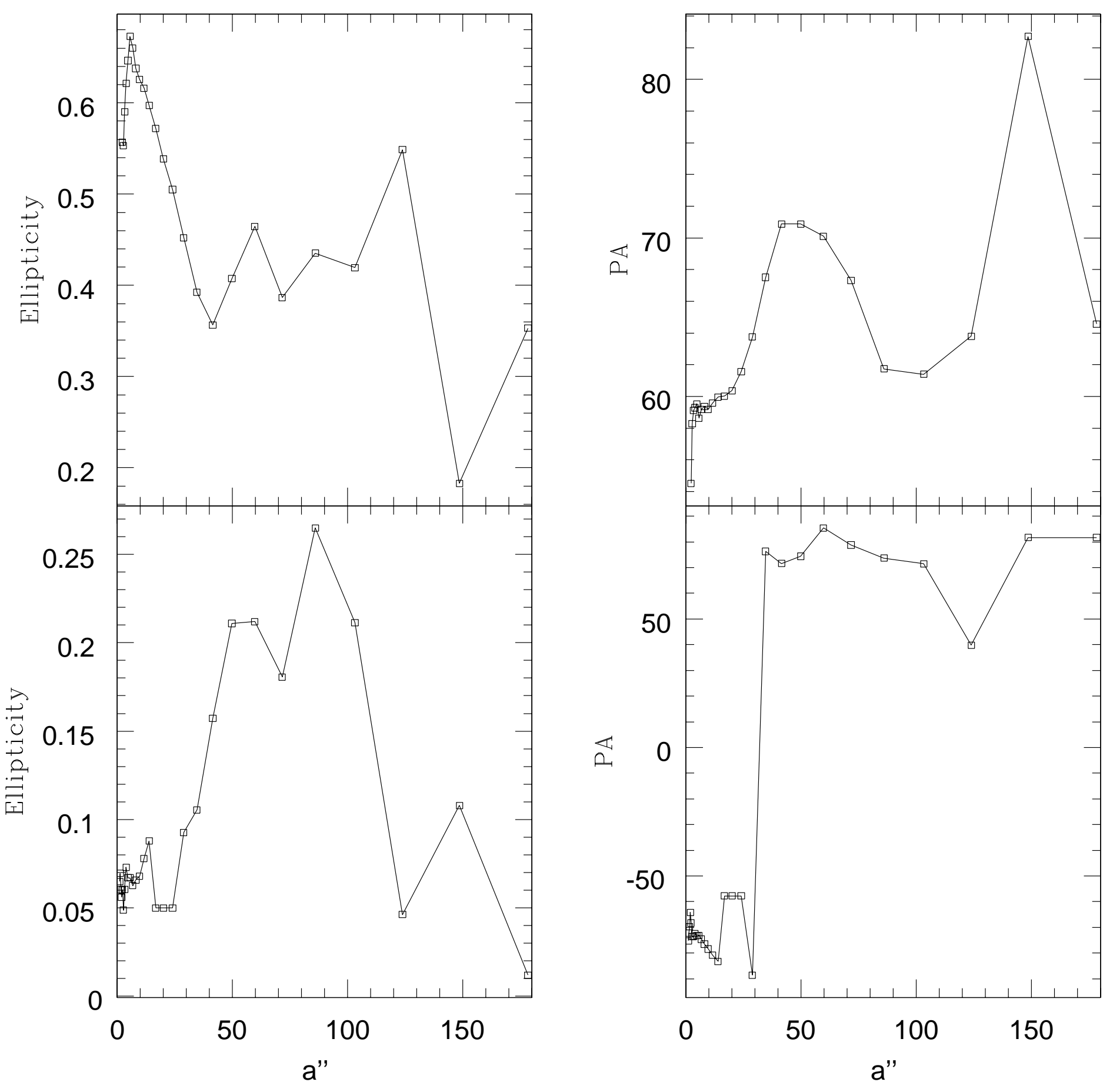
This figure "Rose.fig21.jpg" is available in "jpg" format from: http://arxiv.org/ps/astro-ph/0112346v1 
This figure "Rose.fig22.jpg" is available in "jpg" format from: http://arxiv.org/ps/astro-ph/0112346v1 
This figure "Rose.fig23.jpg" is available in "jpg" format from: http://arxiv.org/ps/astro-ph/0112346v1 


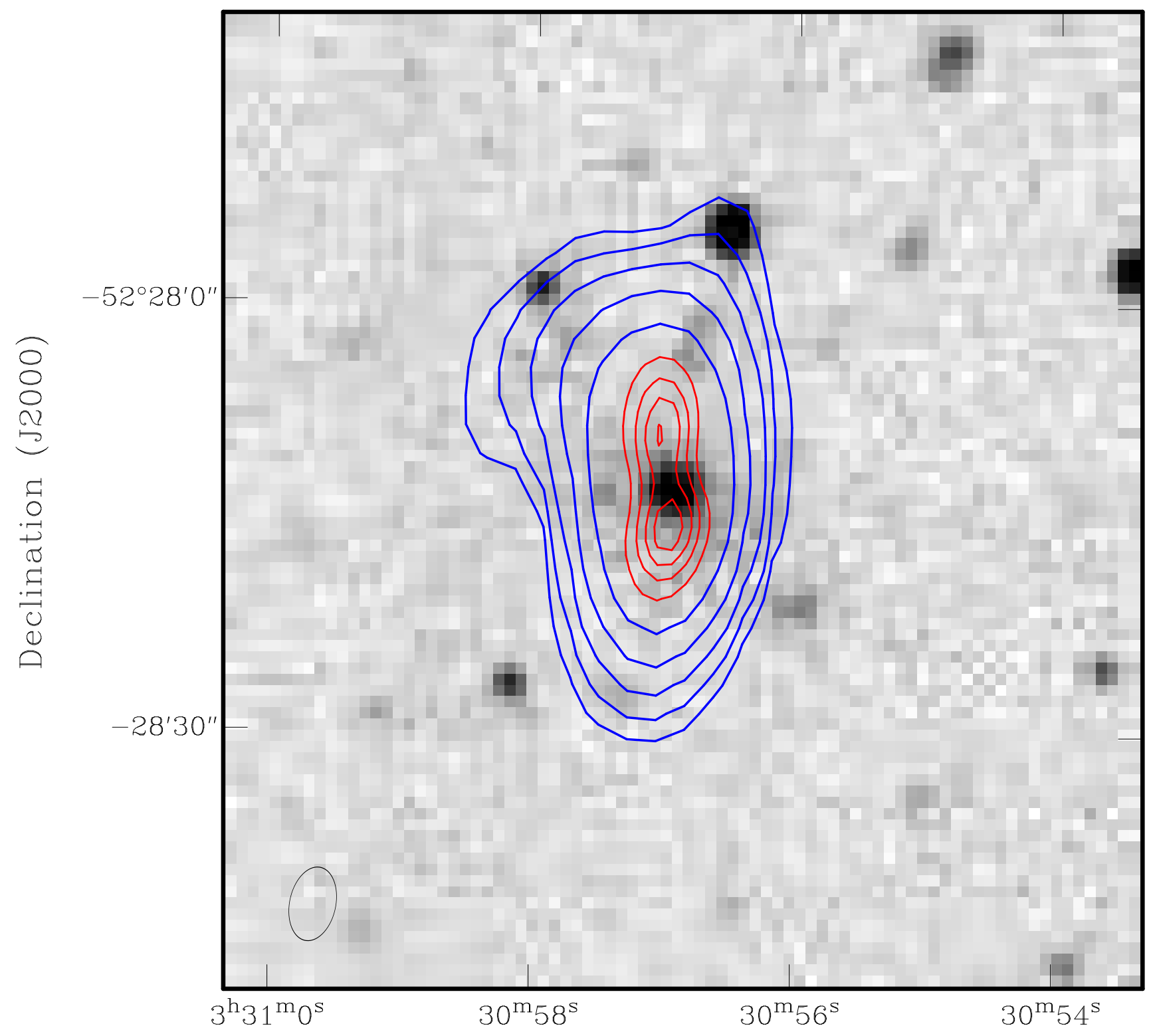

Right Ascension (J2000) 
This figure "Rose.fig25.jpg" is available in "jpg" format from: http://arxiv.org/ps/astro-ph/0112346v1 


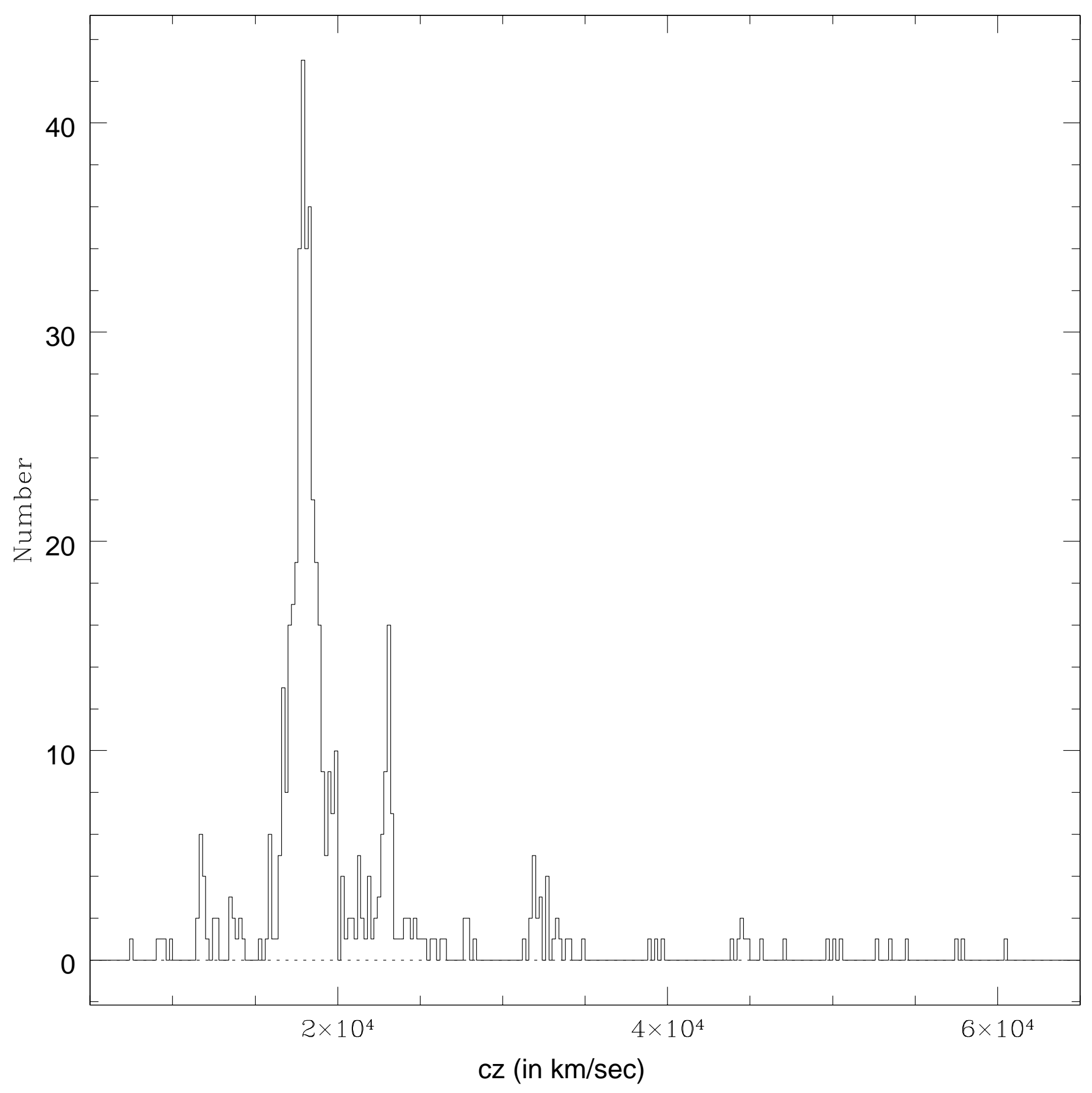


This figure "Rose.fig27.jpg" is available in "jpg" format from: http://arxiv.org/ps/astro-ph/0112346v1 